QL

425

I8S8X

MOLL

3. 


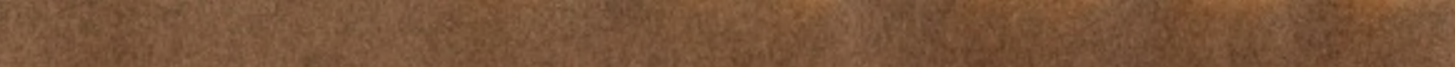
W.

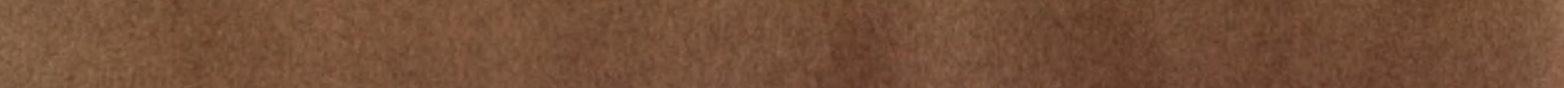
9.

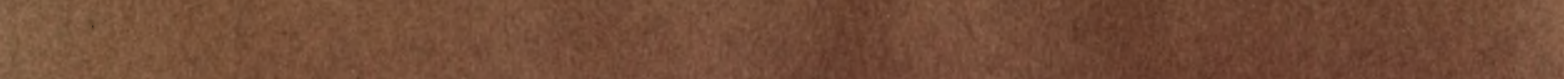
S.

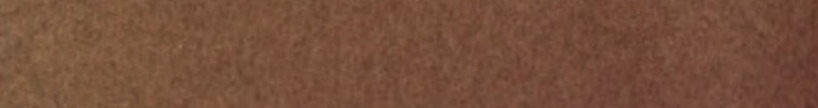

Wotsing

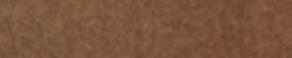

Q

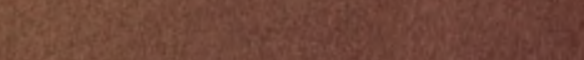

200

(6)

W98.

3.t.

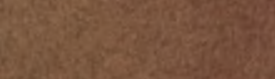

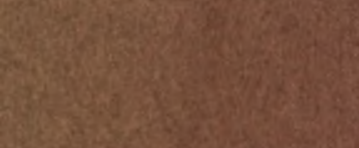

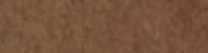

2yoting

(3)

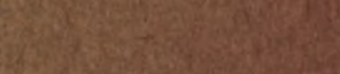

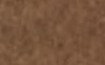

(5)

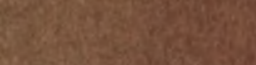

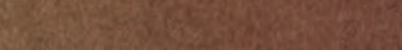

Q.

and

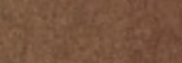

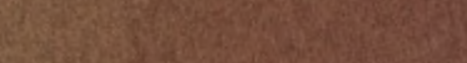

2673 a

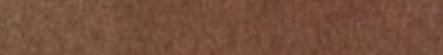

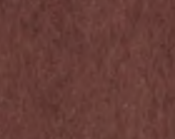

3.7.5.

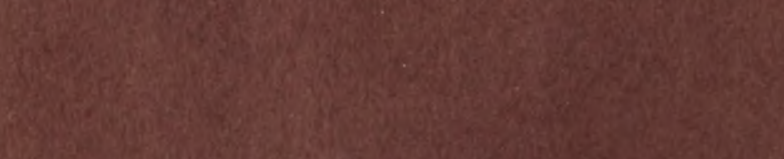

ân.

2.

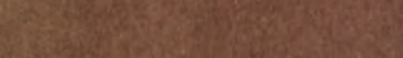

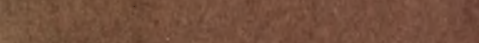


$K-2-d$ STABILE

Division of Mothosile

Sastional tibrary 



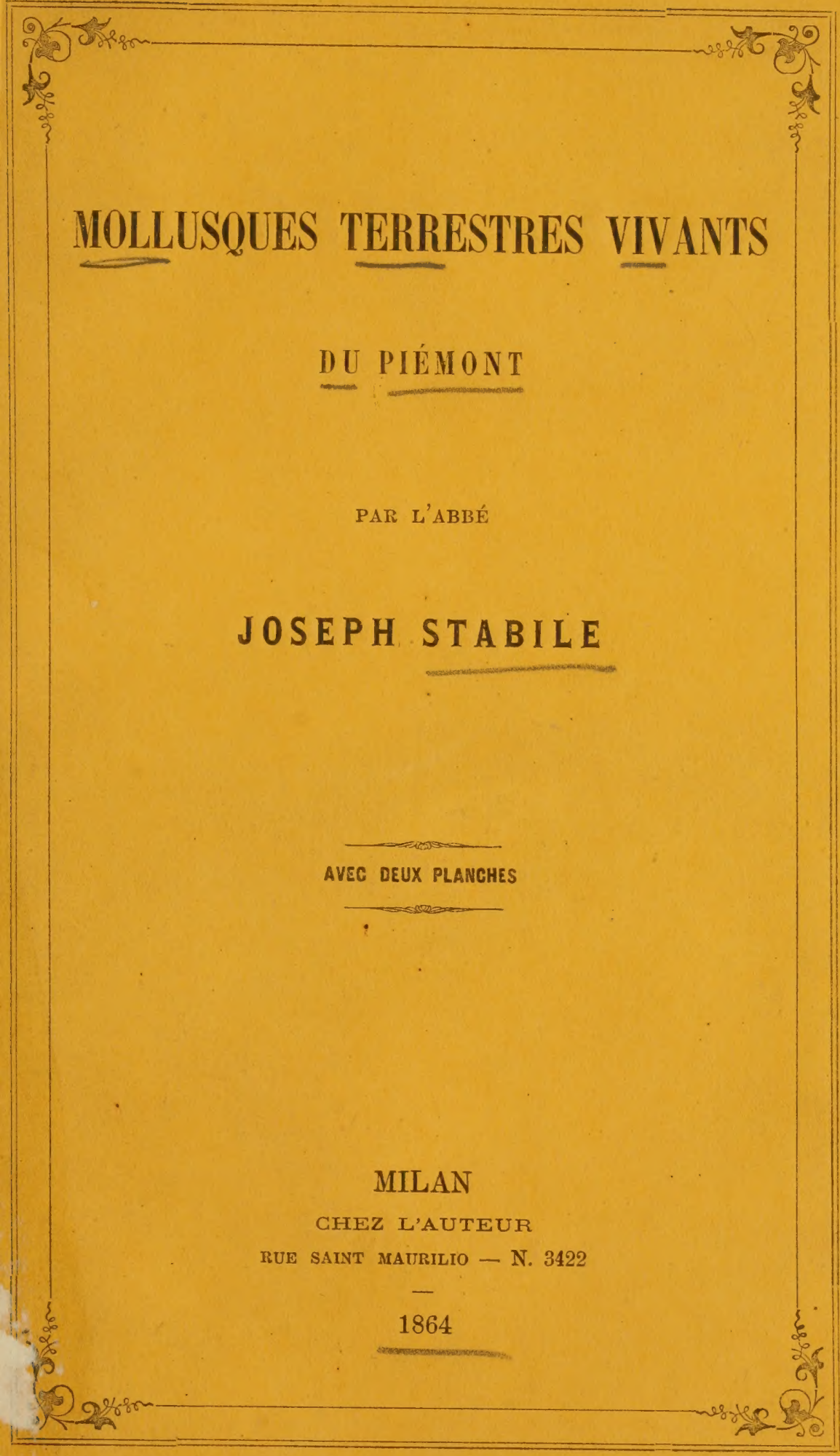



MOLLUSQUES TERRESTRES VIVANTS

DU PIÉ II ONT 

Dal Vol. VI degli Alli della Societi di Scienze Naturali.

(Seduta del 31 gennajo 1864.)

\section{SMITHSON/A, MAY 271957}

LIBRARY 
A LA MENOIRE

DU SAVANT ILLUSTRA'TEUR

DE LA CONCHYLIOLOGIE VIVANTE ET FOSSILE

ET DE LA MALACOSTATIQUE

LE DOCTEUR

\section{J-P-SYLVESTRE DE GRATELOUP}

NÉ A DAX

LE 31 DÉCEMBRE 1789

1)ÉCEDÉ A BORDEAUX

I.E 24 AOÛT $18 \% 1$ 



\footnotetext{
- Una fauna dei Molluscti viventi del Piemonte sarebbo di sommo interesse, perchè, essendo questo posto tra Ja Francia e il Lombardo-Venelo, essa dorrebbe presentarci l'anello di congiumzione tra la fauna occidentale dell' Europa o quella dol mezzodi della sua parte centrale.

\section{P. Sthore, Sui Molluschi viventi del lembo} orientule del Piemonte. (Pavia, 1855.)
}

La Malacologie terrestre et fluviatile du Piémont a été, jusqu'ici, presque tout à fait negligée! C'est M.. Pellegrino Strobel (1) qui nous a donné les premières observations sur la faune malacologique de ce pays. Utilisant ses découvertes et les données d'autres conchyliologistes ses amis (moi, Villa, Marani, Prada, etc.), sous le titre: Molluschi viventi del lembo orientale del Piemonte, dalla Toce alla Trebbia (2), M.: Strobel a publié un catalogue de bien 64 espèces de mollusques terrestres, et 38 fluviatiles du Piémont oriental, c'est à dire de cette partie du Piémont qui est comprise entre la Toce et la Trebbia, la Sesia et le Tessin et la portion de la vallée du Pô à la droite de la Bórmida. Suivent ce catalogue quelques intéressants chapitres dans lesquels l'auteur s'élève à diverses considérations malacologiques et malacostatiques; le dernier mot de M. ${ }^{r}$ Strobel est l'expression d'un vou que d'autres malacologistes viennent augmenter ces faits, et apporter une pierre de plus au grand édifice d'une malacologie italienne. J'ai considéré comme dirigés à moi plus particnlièrement ces encouragements, et après avoir visité plusieurs fois

(1) Professeur d'hist. natur. à l'I'niversité de Parme.

(3) In: Gionnale di Malacologia, par P. Strobel; Pavie, 1853. 
divers points de la Suisse ut de la Lombardie avec le canton du Tessin (1), une partie de la Savoie et le département français des Hautes-Alpes, pour l'étude et la comparaison des faunes malacologiques, j’ai entrepris enfin d'explorer le Piémont. Inutil de dire que j'entends pour Piémont le pays compris entre la Toce, le Tessin et la Trebbia, les Alpes (du col du Simplon au col de Tenda) et l'Apennin boréal.

Comme l'on voit par le titre mème de son ourrage, le tableau de M.r Strobel ne comprend que un petit coin (le nord) de la faune montagneuse et alpine; $\mathrm{j}$ ai donc choisi de préférence à explorer la région occidentale et plus particulièrement les régions élevées, quoique je n'aie nullement negligé ni les collines ni la plaine. Le Simplon et la vallée de la Toce jusqu'à l'extrémité méridionale du lac Majeur, les versants oriental et méridional du Monte-Rosa, les vallées de la Doire-Baltea, de la Stura de Lanzo et ses embranchements, la grande vallée de la Doire-Ripaire avec les endroits reculés du Monte-Thabor, la vallée du Pèllice et l'haute vallée du Pó jusqu'au col des Traverseltes $\left(2500^{\mathrm{m}}\right)$, elc. etc., ces sont les points que j'ai visités et explorés avec la plus grande attention. Tàche rude, travail assez difficile, mais je suis bien heureux de pouvoir ajouter à la liste des mollusques terrestres donnée par M.P Strobel, 42 autres espèces, dont quelques unes nouvelles (2), plusieurs rares ou non vulgaires, et de toutes les 106 espèces terrestres enfin indiquer un bon nombre de localités, et presenter bien des faitg intéressants à l'appui des cludes malacostatiques.

Ainsi, par ex., l'/lelix ciliata depuis l'haute vallée du Tessin ou Val-Lepontina, son limite nord, descend jusqu'à la vallée de la DoireRipaire $(0 u 1 x$, etc.) à l'ovest, et à la Trebbia (Zavatarello,-Nontalto)

(1) Voir ma: Fauna Elvelica: Conchiglic terr. e pur. del Luganese; Lagano 1845-46. et mon: Prospetto sistem. stalist. dei Moll. viv. net territorjo di Lugano; in: Alti Soc. Ital. di Se. nat. Milano; vul. 1. ${ }^{\circ}$, Inglin 1859.

Voyez aussi mes mémoires 1, et II: Sui Fossili del terreno triasico nei dintorni del lago di Lugano in: Actes Soc. Helerit. Sc. nat.; S. Gall. 1854; ct Bâte 1856. of Fossiles des environs du lac de Lugano; in: Act. Soc. Helvet. Sc. nal.; Lugano, 1860.

(2) Coquilles nourelles, ou peu commes; par l'ahbe Ins. Stahile; in: Pirue el Magl. de Znol. par fiuririn Ménicille, Paris, 185. 
à l'est. L'Ilel. nautiliformis des forêtes alpines de la Val-Maccugnaga (versant oriental du Monte-Rosa) côtoyant les bassins des lacs Majeur, de Lugano et de Como, s'abaisse jusqu'aux collines de Viverone près d'Ivréa, et mème jusqu'à la Val-de-Lanzo, à N. O. de Turin. L'Helix glacialis et la Vitrina major appartiennent à tous deux les côtés, savoyard et piémontais, des $\mathrm{Al}$ pes oecidentales; cependant on pourrait regarder la première comme propre plutôt du versant oriental ou piémontais, quoique elle habite aussi les régions élevées du côté occidental (Haule-Maurienne). La Vitrina major, au contraire, est une espèce occidentale ou française qui, descendant du notre côté des Alpes, n'arrive que à peine jusqu'à l'extrémité inférieure de quelques vallées. L'H. zonata habite de çà et de lả de toute la chaîne alpine comprise dans notre tableau, quoique sur le versant occidental et méridional elle soit nulle part abondante, et cela pour la simple raison que cette espèce est propre plutôt des régions alpines boréales que des méridionales. L'IIel. foetens (type) de Studer, a été recueillie dans les Alpes Helvétiques (Vallée d'Entrémont en bas Valais), mais dans cetle localité elle est petite et plus mince que les individus (Var: adelozona) que j’ai trouvés en Piémont (Val-de-Lys, ou Val-Gressoney); plus on va vers l'est (Val-Serina, S. Pellegrino, etc. en Val-Brembana), celte espèce acquiert de dimensions majeures, et son test devient plus épais. L'H. zonata préfère la zone alpine; l'H. adelozona la montagneuse. La Clausilia plicala parait limitée à la seule partie supérieure du bassin du Verbano (Canobbio, Locarno, Bellinzona, Magadino, Luvino), n'arrivant tout au plus que jusqu'à la Toce. La Claus. Thomasiana, avec ses diverses modificalions, depuis le còté occidental inférieur du Verbano descende jusqu'au S. 0 de Ivréa (Castellamonte), et aux vallées de la Stura au dessus de Lanzo, à N. 0. de Turin; la Claus. alpina est le représentant de la $C l$. Thomasiana dans les régions élevées. Quant à la Claus. diodon, qui appartient à la mème section des deux précedentes, elle caractérise assez bien le côté sud du massif du Simplon (1), et plus probablement tout le versant méridional des alpes Pennines!

(1) C'est par erreur que M. ${ }^{r}$ de Charpentier a vile (Cat. Moll. Suisse, 1837) la Claus. dindon de la Val-de-Ragnes en bas Valais. 
La Pupa Ferrarii des ses limites orientaux dans les vallées Brescianes en Lombardie, s'avançant vers l'ovest, cûloye les rives des trois lacs ci-dessus nommés, descende vers le sud sur les coteaux entre Ivréa et Vercelli, s'abaissant jusqu'à la vallée du Tanaro, c'est à dire jusqu'à l'Apennin. Que dira-t-on de la Pupa cinerea recueillic en abbondance par le prof. Mousson (1856), et par moi (1857) a Suse au pied des rochers du Mont-Cenis, et pas ailleurs, en Piémont?

Cinq sont les espèces propres du Piémont, dont trois (Claus. diodon, alpina, et Thomasiana) sont exclusives de la région cispadane (gauche du Pô); une (Pupa Mortilleti), quoique plus propre de la région transpadane, on la rencontre même de l'autre côté (Suse); une (Claus. Mell(e) appartient à la faune transpadane. La faune cispadane est caractérisée en outre par la présence de l'Hel. zonata, fotens, glacialis et nautiliformis, et de la Claus. albopustulata; tandis que la faune au delà du Pô est représentée par les Zonites hydatinus et Leopoldiunus, par l'Hel. cespitum, umbilicaris Var. padana, et conspurcata, et par la Clıus. punctala. C'est encore pour la première fois que j'ai trouvé en Piémont non seulement, mais sur tout le versant méridional des Alpes, la Claus. nigricans, Pulteney (obtusa, C. Pfeiffer) Les quelques individus que jai trouvés en Val-de-Lanzo sont identiques aux types provenants de l'llannover, que je dois à l'obligéance de M.* Adolphe Schmidt; et peu différents de ceux qui m’ont été communiqués par mon ami M. ${ }^{r}$ Turati, recueillis par lui même à Bengor en Angleterre. La Clausilia des diverses localités du côté sud des Alpes, et qui existe dans maintes collections sous les dénominations de Claus, nigricans, Pult.; obtusa, Pfeifper ; dubia, Draparnaud; rugosa, Dhap.; rugosa, C. Pfeiffer, el mème quelquefois avec tous ces noms ensemble rangés en synonymie, n'est d'autre chose que la Claus. dubia, Drap., dont le synonyme est Claus. rugosa, C. Prr. (non Drap.); ou si l'on veut, celle-ci en serait une simple mutation caractérisée par l'avancement et la bifurcation, en dehors, de la lamelle infériéure, tandis que chez la Claus. dubia, Drap. cette lamelle est simple et éloignée. La Claus. perversa, Lissé (Cl. rugosa, Drap。; non C. Prr.) appartient plutòt à la faune littorale (p. e. en llalic: Voltri; en France: Montpellier; en Espagne: Barcelone, ete.). 
Des mollusques terrestres vivants dans la région cispadane du Pié. mont, huit manquent à la Lombardie: Vitrina major; Helix glacialis; Claus. diodon, alpina, Verbanensis, et nigricans; Pupa avenucea (1), et Mortilleti; mais en revanche la Lombardie possède uII bon nombre d'espèces et variélés, presque toutes exclusives: Zonites gemonensis, F Ėr. (sp.); Helix Preslii, F. Sснмıd, var. Luganensis, Scнıмтz; Hel. nisoria, Rossu.; Hel. colubrina, J $\mathrm{JN}_{\mathrm{AN}} \boldsymbol{H}$. frigida, et tigrina Jan; Clausilia comensis, Sнuttleworth; Balsamoi, Strober; clavata, Rossi. (lamellosa, Villa; non W Agner); Leucensis, Vilua; Strobeli, Porro (Stabilei, Charpentier); Villa, Megrle von Munlfeldt; non aliorum (Wathelyana, Charp.); brembina, Strober; exoptata, Ad. Sсhndт; Pomatias Porroi, Strobel; maculatus, Drap., var. Villa, Spinelli.

Le Zonites Leopoldianus, Charpent. (sp.) de la région transpadane, aurait été trouvé aussi à l'extrémité orientale de la Lombardie en Val-Trompia (Spinelli (2)) quoique en exemplaires plus petits, et l'Hel. conspurcata, Drap. sur les collines de Brescia. (Spin.). La Claus. diodon, Thomasiana et alpina, et la Pupa Mortilleti manquent aussi à la Savoie, mais celte région a quelques espèces ses propres: Helix alpina, Faure-Biguet, et var. Fontenillii, Michaud; Pomatias carthusianus, Dupuy (apricus, Mousson).

Une espèce alpine qui ne s'est pas encore présentée, jusqu'ici, en Piémont, est l'Hel. personata, Lams.; et cependant elle habite, non seulement les versants nord et ovest de toute la chaîne alpine, mais aussi (quoique à la vérité assez rare) les méridionales vallées Maggia et Lepontina (Canton suisse du Tessin) (3). Le group des Campylées à test calcaire n'est représenté en Piémont que par une unique espèce (Hel. glacialis), consequence naturelle du peu d'abondance de calcaire sur presque toute la ligne des Alpes, tandis qu'il abonde dans

(1) Il paraît que la $P$. avenacea, Brug. (avena, Drap.) n'existe pas en Lombardie, où cette espece est remplacée par plusieures formes de la $\boldsymbol{P}$. megacheilos, JaN. M. ${ }^{\mathrm{r}}$ Strobel (Essai d'une distribut. etc. Moll. Lomb.) ne cite la P. aven. que du Tyrol italien.

(2) Spinelli G. B. Catal. Moll. della prov. Bresciana; II. ediz., pag. 13, 1856.

(3) Il est bien possible que cette espèce puisse encore être trouvée à l'extrémité supérieure de la vallee de la Toce (Val-Formazza). 
les Pre-Alpes. La Pupa mullidentata, Ouw (variabilis, Duap.) parail manquer au Piemont, et y est remplacée par la jolic 1 . Mortilleti. Mais ce qui est bien frappant, c’est la non présence du genre Pomatias que, ni moi dans nes diverses excursions, ni d'autres conchyliologistes (Strobel, Villa, Mella, Mortillet, I'rada) nous n'y avons pas encore rencontre. Le Cyclost. elegans, assez commun en tonte la Lombardie (jusqu'à l'extrémité inférieure du lac de Lugano) manque à presque toute la région Cispadane, tandis qu'il est très abondant dans la Transpadane (1). Ces sont des faits qui demandent d'ètre pris en consiclération.

Chez les mollusques terrestres, ces petits et cependant non moins merveilleux ètres de la nature, dont le développement, la vie, les mours sont intimément liés aux conditions physiques du sol et metéorologiques, conditions sujeltes à des variations, si non à des altérations importantes, surtout dans les régions élevées; chez les mollusques terrestres l'époque de l'apparition, leur multiplication, leur dispersion, leur habitat présente aussi des irrégularités frappantes, et même des anomalies, si cependant l'on peut ainsi appeler certaines lois économiques de la nature, lesquelles nous ne sommes pas encore arrivés à comprendre, et que, je ne sais pas si plus superbes ou plus ignorants, nous gratifions du nom de désordre; lois qui n'ont jamais cessé d'ètre regles par la très-liaule sagesse du prémier fial qui a liré du caos l'Univers! De nouvelles explorations, done, aux divers points des $\Lambda$ lpes, et plus particulièrement aux versants oriental des Aipes Colliennes, el borial des Mpes Marilimes et de l'Apennin, pourront bien augmenter, peut-etre, la liste des Mollusques terrestres du Piémont, et mème y en ajouter quelque espèce nouvelle pour la faune d'kurope, et enrichir par d'utiles connaissances la malacostatique. Je n'ai pas négligé de faire appel à tous les naturalistes yui, directement ou indirectement, pouvaient me donner des renseignements opportuns, et tous m'ont prèté un bienveillant concours par des intéressantes communications; je citerai, entre autres, MM. ${ }^{\text {r8 }}$ le prof.' Stro-

(1) Un a inulitement essayé de faire acelimater a Parie lu Cycl. clegans. Ses dúpouilles ne sont pas rares dans les alluvions du Tessin, charices par les caux Rezia Imanzio: Gasteropodi torr. e flut. di Pacia; 1818). 
bel, te cher. Gabriel de Mortillet, le eomte Charles Mella de Ver. celli, les frères Ant. et J.B. Villa, le prof. Bellardi etc.; je pric done tous ces Hessieurs de recevoir ici les sineéres remerciments que je dois à leur obligreante bonté. Enfin un mot de gratitude à mon cher frère Philippe-Yarie entomologiste et ornitologiste, et à notre lon ami Antoine Grossi, qui m'ont souvent aidé à la recherche des petits mollusques dans les alentours du lac Majeur.

Je sens la nécessité de donner quelques explications sur la disposition que j'ai adoptée dans cet ouvrage: et d'abord, pour ce qui concerne la distribution zoologico-systématique des espèces, je les ai rangées et groupées suivant leurs affinités anatomiques; quant à la détermination et à la synonymie, je crois d'avoir traité ce sujet avec la plus sérieuse attention. Sur le rapport de la distribution géografique j'ai suivi les divisions qui m'étaient indiquées et tracées par la nature même. La faune malacologique d'un payss assez étendu en longueur tel que le Piémont (1), doit nécessairement ressentir l'influence exercée par le deux opposés points nord et sud; nous avons déjà fait observer que la grande rivière du Pò marque une ligne de séparation entre deux faunes, l'une septentrionale, méridionale l'autre; j'ai donc divisé la fanne malacologique piémontaise en cispadane et transpadane. Mais ce n'est pas tont: la faune des vallées et celle de la plaine, sont (sauf quelques exceptions) nettement distinctes; si telles ou telles autres espèces planicoles s'élèvent dans les régions élevées, il n'arrive si non exceptionellement, que quelque espèce monticole franchisse la limite inférieure de la vallée pour s'élablir dans la plaine; quoi qu'il en soit, quelques exceptions ne détruisent pas les lois générales. Une division donc en faune des vallées et faune de la plaine élait dictée par le simple raisonnement. $L a$ grande plaine du Pô (laquelle, pour les motifs que nous avons déjà exposés, il faut aussi diviser en cispadane et traspadane), s'élève plus ou moins brusquement en deux sens, c'est à dire de est à ovest en mème temps que à nord at à sud; le plus bas point de son incli-

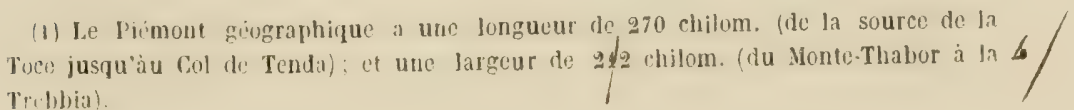
Trubial. 
nation est a $41^{\text {tr }}$ (a l'embouchure de la Trebbia dans le Pó). A partir de ce point la plaine cispadane s'élève, par le Tessin, jusqu'à $200^{\mathrm{ml}}$ (lac Majeur); par la Sésia, de $9 \mathrm{5}^{\mathrm{m}}$ (embouchure dans le Pó), à $280^{\mathrm{m}}$ (près Gattinàra); par la Doire-Bältea, de $117^{\mathrm{m}}$ à $240^{\mathrm{m}}$ (inférieurement à Ivréa); par l'Orco, de $123^{\mathrm{m}}$ à 400 (à Livarolo); par la Stura de Lanzo, de $138^{\mathrm{m}}$ à $450^{\mathrm{m}}$ (entre Cirié et Lanzo); par la Doire-Ripaire, de $137^{\mathrm{m}}$ à $330^{\mathrm{m}}$ (Rivoli, etc.); par le Péllice, de $188^{\mathrm{m}}$ à $370^{\mathrm{m}}$ (un peu au dessus de Pinerolo). La plaine transpadane s'élève de $41^{\mathrm{m}}$ jusqu’a $280^{\mathrm{m}}$ (région padane proprement dite, de la Trebbia à Saluzzo); et par la Maira, jusqu'à $600^{\mathrm{m}}$ (près Dronéro); par la Stura de Cuneo, jusqua'à $450^{\mathrm{m}}$ (Cuneo); par le Tànaro, de $82^{\mathrm{m}}$ à $800^{\mathrm{m}}$ (Mondovi). Les collines de l'Astésan sont comprises, mème pour la faune malacologique, dans la plaine padane. Mais du cùté de l'Apennin, la plaine transpadane n'est d'une quelque étendue que entre la Bórmida et la Scrivia, jusqu'aux coteaux du Tortonois et à ceux qui forment les courtes et petites vallées subapennines situées entre la Scrivia et la Trebbia.

Je ne ferai pas ici l'énumération des ouvrages que j'ai consultés sur l'anatomie, la comparation des espèces, la discussion de la synonymie, etc.; je citerai seulement ceux qui ont quelques rapports avee la malacologie et la malacostatique du Piémont.

Après de fatigantes et pénibles excursions, et de longs et séricux études, qu'il me soit permis d'espérer que mon travail sera favorablement accueilli parmi les savants culteurs de la malacologie.

Milan, Mars 1864.

Abbé Juseph Strabite 


\title{
OUVRAGES CONSULTES
}

\author{
QUI ONT DES RAPPORTS
}

AVEC La FaUNe Malacologique dU PIÉMONT

\section{a). pour la Malacologie}

Charpentier (Jean de). - Catalogue des Mollusques terrestres et fluviatiles de la Suisse. Neuchâtel, 1837 ; avec 2 planches.

Moqun-Tandon (Alfred), - Histoire naturelle des Mollusques terr. et fluv. de France. Paris, 1858 ; avec 84 planches.

Mortillet (Gabriel), et Dunont (Francois). - Hist. des Mollusques terr. et d'eau douce vivants et fossiles de la Savoie et du bassin du Léman; in: Bulletin de la Soc. hist. natur. de Savoie; 1882-83-\$4.

Mortilete (Gabriel), et Dumonr (François). - Catalogue critique et malacostatique des Mollusques terr. et d'eau douce de la Savoie et du bassin du Léman; in: Bulletin de l'Institul Genevois. Genève, 18 ธ7.

Mortillet (Gabriel). - Annexion à la Faune malacologique de France; in: Révue Savoisienne. Annecy; février, 1861.

Montillet (Gabriel). - Étude sur les Zonites de l'Italie septentrionale; in: Atti della Soc. Itat. di sc. nat., Vol. IV; Milano, novembre, 1862 .

Spinelu (Gio. Batt.). - Catalogo dei Molluschi terrestri e fluviali della provincia Bresciana. Brescia; 1881, con una tavola.

Spinelu (Gio. Batt.). - Catalogo, etc. 2. adizione corretta ed accresciuta. Verona, 1856, con una tavola.

Stabule (abate Giuseppe). - Prospetto sistematico-statistico dei Molluschi terrestri e fluviali viventi nel territorio di Lugano (Svizzera italiana); in: Atti della Societa Geologica ital., vol. I. ${ }^{0}$ fasc. III; Vilano; luglio, 1889. 
Sirnale (abbe Joseph). - Description de quelques coquilles nouvelles ou peu connues; in: Révue et Magas, de Zoologie, par Guérin- llénéville; Paris, aout, 18B9, avec une planche.

Srnober (Pellegrino). - Sui molluschi viventi del lembo orientale del Piemonte, dalla Toce alla Trebbia; in: Giornale di Malacologia compilato per P. Strobel; Pavia, Anno I. , 1883.

Stroder (Pellegrino). - Essai d'une distribution orographico-géographique des Mollusques terrestres dans la Lombardie; in: Mém. Acad. des Sciences de Turin; Série II, tom. XVIII, 1837.

Vicla (Antoine et Jean-Bapt.). - Catal. dei Molluschi della Lom. bardia; in: Notizie naturali e civili su la Lombardia per Carlo Cat. taneo; Milano, 1844.

\section{b). pour lı Malacostatique.}

Catraneo (Carlo). - Nolizie nalurali e civili su la Lombardia; Milano, 1844.

Chabrol (Comte Gilbert de Volvic) - Statistique des Provinces de Savone, d'Oneille, d'Acqui et de Mondovi. Paris, 1824.

Saluzzo (Annibale di). - Le Alpi che cingono l'ltalia considerate militarmente, etc. Parte 1. ${ }^{\circ}$ Le Alpi; Torino, 1848.

Ziegler (J.). - Ilypsometrie der Schweiz; Zürich, 1883.

Zuccagni.Orlandinı (Altilio). - Corografia fisica, storica e statistic:a degli Stati Sardi Ltaliani di Terra ferma; Firenze, 1833.

NB. L.es especes signées far - sont celles cities par M.P Strobel dans sons ourrafe: Sui Molluschi vienti del lembo orientale, ete. 


\section{PULMONATA}

I Stylommatophora, sive oculos in apice pedunculorum ferentia.

A. PULmONATA INOPERCULATA. (1)

Familia: L I M A C E A.

Gen. ARION. (2)

(Arion, Fèrussac - Hist. Mollusq. 1819, pag. 50-53.)

Sect. L O C H A. (3)

(Lochea, Morquin-Tandon - Hist. Moll. de France; 1853.)

* 1. Arion rufus. (4)

Zimax rufus, Linné - Systema Nalurce; Édit. X, 1758.

Région Padane. - Plaine du Po (nord): rives boisées du Gravellone au sud de Pavie, š 7-100 mètres (Prada).

\section{Arion cinctus.}

Limax cinclus, Müler - Vermium Historia, 1774, Vol. 1I, pag. 9.

- subfuscus, Draparnaud - Hist. Moll. 180э, pl. 1X, f. 8; non C. Pf́eiffer.

Vallées Gispadanes. - Vạllée de la Toce: Hospice du Simplon, $2020^{\mathrm{m}}$ (Stabile) - V. de la Doire Riparia: Mont-Cenis, $1900^{\mathrm{m}}$ (Strobel).

(1) Voir ces Notes à la fin du Jiurc. 
R. P. - Haute vallée du Po: audessus du village de Crisolu $1600^{\mathrm{m}}$ (Stabile). Sous les pierres crystallines parmi les gazons.

\section{Sect. IL L LEI S. (s)}

(Prolepis, Mor. Tamiun. - loco cilutuj.

\section{Arion hortensis.}

Arion hortensis, Firussac - Hist. Moll. 1819, page. 65.

VAr: ๙) Alpicola, Pér. - loco cilato, pl. Vill, A; lig. $\unlhd-4$.

V. C. - Val de la Sésia: Notre-Dame d'Orópa, 430m. Sums lest pierres de micaschiste (Mella).

\section{Gen. LIMAX.}

Limax (partim), Linni- System. Nul, , luco cilulo:.

Sect. M A L I. $(b)$

(Amulia, Mor. Tanil. - li)co cilato, par. 19:.

\section{Limax marginatus.}

Limax marginalus, Muller - Verin. hist. 1774, Il. ; Moruin-Tans. - loco ciluto pl. II. lig. 4.

R. P. - Haute vallée du Po: Ćrisolo, $1400^{\mathrm{m}}$ sous les pierres crystallines (Stabile). - Plaine du Po (sud): Montallo, Castegrgio en Val Coppa, 46-100 (Strobel).

(a) Cuirasse recuurant une limacelle imparfaite, rugueuse, qui semble frutuite par l'agrigation d'un certiin nombre de granulations calcaires.

(b) Cuirasse pourvue de rugositis plus ou moins apparentes; stries contentriques nulles. 
Vallees Transpadanes. - Val-Varaita: Venasca, $359^{\mathrm{m}}$ (Morsillet).

\title{
Sect. E U I I M A X $(a)$
}

\author{
(Eulimax, Hoq. Tindon - loco citato pag. 22).
}

\section{* 2. Limax agrestis.}

Limax agrestis, Linnes - System. Nat. Élit. X, 1758; 1, pag. 632.

«) Filans, Hoy - ill: Transacl. Limi. I, 1791; Férussac - Hist. Holl., pl. V, fig. $9 \cdot 10$.

V. C. - Val Sesia : environs de Vercelli, $150^{\mathrm{m}}-$ V. de la Doire Bàltea: Viverone, $\mathbf{3 3 0}$ (Nella) - V. de la Doire Riparia: Nont-Cenis, $1900^{\mathrm{m}}$ (Strobel).

R. P. - Haute vallée du Po: Paesana, $\$ 40^{\mathrm{m}}$ (Wortillet) - Plaine du Po (nord): Turin, 220-250 (Vort.); Lomelline, $100^{\mathrm{m}}$ (Strob.) Plaine du Po (sud): Monte Pénice en Val-Tilone, $30-300^{\mathrm{m}}$ (Strobel).

3) ReticuIatus, Müller - Verm. Hist. 1774; Férussac - Hist. Moll. pl. V, fig. $7 \cdot 10$,

V. G. - V. de la Doire Rip.: audessus de Clavières (route du Mont Genève), $1800^{\mathrm{m}}$ (Strob.); Bardonnêche, $1530^{\mathrm{m}}$ (Strobel).

R. P. - Plaine du Po (nord): rives du Tessin (Strobel); (sud): Valmadonna près d'Alexandrie, $120^{\circ}$ (Strobel).

V. T. - V. de la Staffora: Varzi (Strobel).

\section{Limax arborum.}

Limax arbortm, Bunchard-Chantereiux. - Mollisq. Pas-de-Culais, 1835.

V. C. - Val de la Toce: Simplon, $2000^{m}$ (Stabile, Strobel).

(a) Cuirasse a stries roncontriques plus ou muins distinctes: rugusitis nulles ou mosulue nulles. 


\section{* Limax variegatus.}

Limax variegalus, Draparnaud - Tableau des Mollusq. Isiol; Firussat - Hist. Moll. 1819 , pl. Y, ffg. 3 .

R. P. - Plaine du Po: rives boisées du Tessin, 86-80m (Strobel).

V. T. - V. de la Trebbia: Bobbio, $863^{\mathrm{m}}$ (Strobel).

Obs. Celte espece (nocturne) doit être assez répandue, surtout ì la plaine. Il n'est pas rare de la rencontrer mème à Vilan dans quulques caves.

\section{* 5. Limax maximus.}

Limax maximus, Linne - System. Natur, Fillt. X: 1758, 1, pag. 652.

- cinereus, Muller - Verm. Hist. 11: 1774, pag. 5.

a) Cellanious, drargenville - Conch. pl. Xxill, fig. 31 : Ferussac - Mist. Moll. 1819, pl. IV, fig. 8. Cinereus; clypeo, dorsoque muculis nigris lfascias in. termptas in dorso simulantibus).

V. C. - V. de la Toce: Vogogna, et autres endroits de l'Ossola, 190-300 ${ }^{\mathrm{m}}$ (Stabile) - V. de la Doire Bàltea: collines de Viverone pris d'Ivrea $330^{\mathrm{m}}$ (Mella).

R. P. - Plaine du Po (nord): Vercelli, $150^{\mathrm{m}}$ (Mella) - Lomellina (Strobel); plaine du Po (sud): Saluzzo, $360^{\text {in }}$ (Mlortillet).

V. T. - Val Coppa: Borgopariolo (Strobel).

B) Rufescens, Moguin-Tandon: - Moll. de France, 1855; pag. 29. "Rufescens, carina el mis mabis. Strobel: (Holl. del lembo or: del Piemonle, ete)

R. P. - Guasta, Lomcllina (Prada). 


\title{
6. Limax cinereo-niger.
}

\author{
Limax cinereo-niger, Wolf - in: Sturm: Deutschl. Fauna; Würmer, 1. fuse, 1803. \\ - anliquorum, Var. $a$, Férussac - Hist. Moll. 1819; [1]. IV, fig. 1. \\ - coenulans, M. Bielz (fide Strubel) - E. A. Bielz: Land und Sïssw. Monl. Sie. \\ benbürgen: 1860. - Voir pour I'anatomie: Adolphe Schmidt - Geschlechts. \\ apparal der Stylommatophoren, 1855 ; pl. XIII, lig. 100.
}

L. margnus, corpore ruyoso, rugis longiludinalibus distinctis; supra plus minusve nigricanti-fusco, scepius longitudinaliter quadrifasciato, lateribus pallidioribus; solea sub-albida, interdum ni. gro limbata; clypeo mediocri, postice obtuse angulato, nigricanti; carina albescenti, distincta, veluti crispata, dimidiam dorsi longitudinem sub-rpquante.

Animal grand, de la taille du L. maximus, oblong, subcylindrique, assez épais, faiblement tronqué et un peu rétréci antériéure. ment, diminuant insensiblement et pointu en arrière. Corps noirâtre ou brun-noir, sur le dos, plus clair sur les flancs, souvent avec quatre bandes longiludinales noiràtres ou brun-foncé; couvert de rides longitudinales dont les intervalles ou rugosités sont saillantes, très-fortes et allongées. Con, ainsi que la tête, les peduncules oculigères et les tentacules, brunàtres; le prémier est verruqueux, et marqué de deux sillons longitudinaux. Cuirasse médiocre, terminée obtusement en arrière, noiràtre ou brune-pâle, souvent avec des taches brunes sur un fond blanchâtre vers les hords latéraux. Pied non frangé, à còtés étroits. Carène d'un blanc-jaunàtre, comme crénelée, saillante ver's la partie postérieure et s'avancant jusqu'à la moitié du dos. Sole blanc-jaunâtre, quelquefois bordée longitudinalement d'une large bande noiràtre.

Limacelle minec, fragile, aplatie, à peine convixe.

Long. 10-17 cenlimètres; Larg. 13.20 millim. 
V.1R. I. Albipes - Sulea uniculare, albido-lutea: pedis nargine externo rigricanli, tel non. (L. lineatus; var. albipes, Dumunt et Murtillet: Mist. Moll. terr. at d'ean douce viv. et foss. de la Savoie et du bussin du Liman, 1852).

a) Inllile luteus, confuse quadrifusciatus, fuscomaculatus; elypeo, dorsoque, medio valde fuscalis, hand raro nigricantibus.

B) Pallide lutens, lateribus fusco-maculatus, maculis ad marginem clypei fasciis duabus linearibus subparalelis effurmantibus: clypeo autem, supra, vabde fusca(1); postice, in area lutea, macula quadrangulari brmmen omato; dorso distincte quadrifasciato, fasciis mediis angustis, exterioribus lutis.

v) Lnicolor, nigricanti-fuscus; antice (sub clypeo) albido-lutescens, clypeique lateribus externis pallidis, vix luleo-maculatis.

VAR. II. Nigripes - Solea late nigro-marginala (Limax (Arion) lineatus, Dumont - Bullet. Soc. IIist. nat. de Savoje; 1849).

b) Niger; carina nigra, breviori; sub clypeo albido-lutescens; clypeo nigro, vix ad marginem externum punctis pallidis sparso (L. ater? Razoumowsky Hist. nat. du Jorat, etc. 1789).

V. C. - V. de la Doire Liparia: Mont-Cenis, $2000^{\mathrm{m}}$ (Strobel) - Var. I. (a)

- V. Anzasca entre Calasca et Pestarena, $1000^{m}$ (Prada) - Var. I, el II. (l)

R. P. - Haute vallée du Po: Calcinero, $700^{\mathrm{m}}$ (Stabile) - Var. I, $\alpha$. Trois exemplaires pru variés l'un de l'autre, si non par la couleur plus ou moins brunatre ou brun-noirâtre. Long. 16-17 centim. Sous les grosses pierres (cristallines) dans un petit vallon ombragé et frais. Hucus épais, un peu vitreux. - $\Lambda$ u dessus de Crisolo (1400 j’ai trouvé la Var. II, ô. Un seul individu, long seulement de 8 cent. larg. 10 millim. (jeune?). Je le rapporle provvisoirement à la du $L$. cinereo-niger, mais la carene assez cotorte (tandis que même daus les jeunes individus du $L$. cinereo-niger la earene monte jusqu'à la moitié du dos) serait suffisant pour l'en separer, de méme que son

(a) Limax carulans, liielz (Strobel).

(b) L. cinereus, Mull.: Van: solea nigro-limbata; rel cincrea, nigro-maculala (Strobel). 
corps qui est, proportionellement, plus grèle. - Plaine (sud) du Po: Cicognola, Stradella (Strobel).

V. T. - Vallée du Gesso: Col de Tenda, $1870^{\mathrm{m}}$ (Mortillet) - Var. I. $\beta$. et $\gamma$.

Obs. M. ${ }^{\mathrm{x}}$ de Mortillet dit que la VAr. albipes est très rare dans les montagnes et aux environs de Bonneville; mais $\mathbf{M}^{\mathrm{r}}{ }^{\mathrm{r}}$ Brot de Geneve assure qu'elle est commune à Vétraz-Nonthoux; toutes deux les localités citées appartiènnent au hassin de Bonneville, Vallée de l'Arve, en Savoje. M. ${ }^{x}$ de Mortillet, qui a traversé plusieures fois le Col de Tenda, jusqu'ici n'a pas encore rencontré sur le versant nord, la varielé à sole bordée de noir.

\section{Famila: H E L I C E A.}

\section{Gen. VITRINA.}

(Fitina, Drapartaul - Tabl. Noll., 1801.)

Seet. SEM II M X. $(a)$

[Semilimar, Stabile - Deseript. Coq. stoutelles, ou peil connus, in: Rícue et Hag. Zuol. par Guirnn; Août, 1859.)

\section{* Vitrina diaphana.}

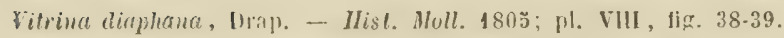

Melicolimax vilieu. Férussac - Tabl. Noll. 1822 ; et Hist. Moll. pl. IX, firg. 4; MuquinTaublon - Moll. de France, 1855 , pag. 46-47 (animal).

V. C. - V. de la Toce: Hospice du Simplon, $2020^{\mathrm{m}}$ (Stabile) - V. de la Doire Bállea: l'Allée-blanche, versant ilalien du Mont Hanc, $2000^{\mathrm{m}}$ (Dumonl; Courma eur, $1218^{\mathrm{m}}$ (Bellardi). Plus vers le nord (Alpes Suisses) celle espèce descende jusquà $960^{\mathrm{m}}$ (Val-Leponlina, versant italien); ct mème dessous de $900^{\mathrm{m}}$ (versant nord, ou Suisse).

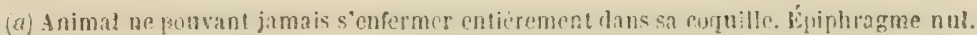




\section{Vitrina Charpentieri.}

Tilvina glacialis?, Forbes - Jurd. ann., 1837.

- - - Charpenticr - (olim), in specim.

- nivalis, Charp. - (postea), in specim.

- - _ - in: Dumont et Nurtillet: Hist. Moll. Savoje; in: Bullet. Soc. hist. nat. Saroje; 1852-53.

- glacialis, et nivalis, plerumq. auct.

Vitrina Charpentieri, Stabile -- Descript. Coq. nouvell. on peu comues, in : Révuc, el Mag. Zool. par Guirin N. 7, 1859 ; fiģur.

V. C. - Vallée de la Toce: Ilosp. du Simplon, $2030^{\mathrm{m}}$ (Stabile) - V. de la Doire Bàltea: Petit Saint-Bernard, $2000^{\mathrm{m}}$ (Fr. Dumont) - V. de la Stura de Lanzo, $1900^{\mathrm{m}}$ (Stabile). Dans tous ces localités: sous les pierres crystallines et préferablement près des ruisseaux alpins. Comme je viens de dire dans mon memoire (Descr. Coq. nouv. etc.) c'est pour trancher toute question, que $\mathbf{j}$ 'ai mis de coté les noms de nivalis et glacialis, et j'ai nommé cette jolie espèce du nom de l'ilIustre de Charpentier, qui l'a trouvée pour la première fois sur les Alpes de Béx dans le Canton de Vaud.

\section{Sect. II E N C O I M A X. ( $(a)$}

(Phenacolimax, Stabile - Coq. nour. elc.. in: Revuc de Guírin. L. cit., 1859.)

\section{Vitrina major.}

Helicolimax major, Firussac pire - Essai mithod. conch., 1807.

Fitrina pellucida, Draparnaud - Tabl. Moll., 1801; et Hist. Moll. 180ä, pl. VIll, $\mathrm{jig} .34-37$, (non Vilr. pellucida, Müller).

- Drnparnaldi, Cuvier $(b)$ - Rigne animal; 1817. II, nule; Moquin-Tand. - Moll. de France 185\%; par. 49, pl. VI, fig. 14-32 (Anatum.).

V. C. - Vallées du Cerro et de l'Elvo: Sanctuaire de N. D. d'Oropa, $1280^{\mathrm{m}}$ (Cesali); - Val de Lanzo: un heure au-dessus de Lanzo

(a) Animal pouvant senfermer entierement dans sal enguille. lipiphragme vitreux.

(b) Conne rectius Drapamaudi, a Jiaparnamet? 
jusqu'au plan de la Mussa, $850-1850 \mathrm{~m}$, sous les pierres crystallines, près les ruisseaux (Stabile) - V. de la Doire Riparia: alpes au-dessus du village de Rochemolles au Monte-Thabor, $1900^{\mathrm{m}}$ (Stabile).

Obs. Dans les régions inférieures des vallées, les exemplaires sont petits, mais dans les régions elévées ils atteignent tout leur développement (Long. 7 millim.). Je ferai même observer que les individus que j’ai trouvé dans les localités ci-dessus nonımées, ont tous le test, en dessus, un peu déprimé; tandis que les quelques échantillons provenant de Montpellier et de l'Aube, que je dois à l'obligeance de MM.rs de Charpentier et de Saint-Simon, sont un peu plus convexes.

\section{* Vitrina pellucida. (5)}

Vilina pellucida, Müller - Verm. hist. II, 1774 (n $\mathrm{n}$ n V. pellucida, Drap.).

Helix limacoides, Alten - System. abhandl., 1812; pag. 85̆, pl. XI, fig. 20.

Titr. subglobosa, Michaud - Complément à Drap. ; 1831, pl. 15, fig. 18-20.

V. C. - Val-Anzasca (Monte Rosa): Alpe du Filar, près du glacier 1700-1900 (Stabile), - V. de la Toce: Hospice du Simplon, $2015^{\mathrm{m}}$ (Stabile) - V. de la Stura de Lanzo, 1800-1900 ${ }^{\mathrm{m}}$ (Stabile). Dans toutes ces localilés: sous les pierres crystallines près des ruisseaux, avec le Zunites fulvus, la Pupa triplicata, la Claus. alpina, etc. - V. de la Doire Riparia: plain du Mont Cenis, $191 \mathrm{~B}^{\mathrm{m}}$ (Mortillet, Strobel).

R. P. - Haule vallée du Po: alpes de Crisolo, $1600^{\mathrm{m}}$ (Stabile); plaine du Po (nord): rives boisées du Tessin, 60-100 (Strobel); plaine (sud): Valmadonna près Alexandrie, $80-100^{\mathrm{m}}$ (Strobel).

V. T. - Apennins (Strobel).

Obs. C'est la seule espèce de Vitrina qui des régions les plus elevées $\left(2100^{\mathrm{m}}\right)$, déscend à la plaine $\left(100^{\mathrm{m}}\right)$. Épiphragme vitreux, quelquefois irisé, proportionellement assez solide, très adhérent. 


\section{Vitrina annularis.}

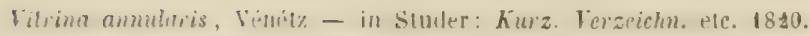

Heliculimax anmularis, Firussac - Hisl. Moll., pl. IX, fig. 7.

V. C. -- V. de la Toce, au-dessus de l'hòspice du Simplon, $2100^{\mathrm{m}}$; sous les pierres crystallines pres des ruisseaux et parmi les herbes et les gazons (Stabile) - V. de la Stura de Lanzo: au-dessus de Balme, $2000^{\mathrm{m}}$ (Stabile).

R. P. - Ilaule vallée du Po: pâluragges alpins au dessus de Crisolo, sous les grosses pierres crystallines, $1600-1800^{\mathrm{m}}$ (Stabile) - Assez rare partout.

\section{Gen. SUCCINEA.}

Sucrinca, Draparnaur - Tableau des Mollusques : 18011.

\section{* Succinea putris.}

Ilelix patris, Liune - System. Nature : - Litit. X, 1758: pag. 774.

Succinua amphibia, Inap. - Tabl. .Holl. 1804.

R. P. - Plaine du Po, rives du Tessin, prairies et lieux marécageux (Strobel, cte.).

V. T. - V. de la Treblia: Bobbio, (Strobel).

\section{Succinea ivfelfferi.}

Succinea Pfeifferi, Rossmässler - Ronog. d. Land-umd Susst. Moll. , 1: 183 ว.

2) Mediolamensis, Villa - Catal. Holl. Lombard. 18\%4: - Slahilr: Prosp. Sistem. Slatist. Moll. di Lagano; 1839 , pag. 17.

R. P. - Plaine du Po (nord): Vercelli, $180^{\mathrm{m}}$ (Mella): (sud): Asti. $154^{\mathrm{m}}$ (Mellil). 
F) Mortllleti, mihi - (De la mêne taille $(a)$, et furme de la S. metliolanensis; elle en diffire cependant par la spire qui, chez les individus adultes, est un peu plus raccourcie, ce qui rend l'ouverture plus courtement ovale. se rapprochant quelyue peu de celle de la Succ. oblonga. Nutre S. Mortilleti a l'onverture plus perpendiculaire a l'axe que chez la $\mathbf{S}$. mediolanensis).

V. C. - Mont Cenis, $1900^{\mathrm{m}}$; sur les plantes aquatiques dont la base se trouve an bord du lac (Nortillet).

\section{Succinea dehracea.}

Snccinea ochracea, De Bella - Nalucol. della Talle di Non, 1852; pl. 1, fig. 1.

V. C. - Simplon (Strobel).

\section{* 4. Succinea oblonga.}

Succinea oblonga, Draparnaud - Tabl. Moll. 1801 ; et Hist. Moll.1805, pl. 1I, fig. 24-25.

R. P. - Rives du Tessin (Strobel).

B) Dromëts, Dumont et Murtillet - Catal. crit. et malacostat. Moll. Savoje, elc. ; in: Bullet. Instilut. Nation. Généxois, 1857, pag. 26.

(Un peu plus grande que l'oblonga el l'arenaria, elle s'en distingue par sa spire plus obtuse et plus ventrue qque la première; plus allongée et plus oblique que la seconde; l'uuverture s'unve plus en dehors de l'axe.

V. C. - V. de la Doire Riparia: Mont-Cenis, au bord du lac, $1910^{\mathrm{m}}$ (Mortillet).

y) Inumblis, Drouët - ICoquille assez petite, ouverture plus arrondie; intermediaire entre l'oblonga et l'arenaria, mais plus rapproche de cette dernière).

V. T. - Vallée de Varaita : Brossasco, $600^{\mathrm{m}}$ (Mortillet).

a) Hautcur 9 à 11 ; Diam. \& 1, i 5 is millin. 


\title{
5. Succinea arenarla.
}

Surrinen arenuria, Bouchard-Chantereaus - Moll. du Pus-de-Caluis, 1838; Moquin Tandon- IIist. nat. Holl. de France, 1855; jag. 62, 11. III, fig. 34-36.

V. C. - V. de la Stura de Lanzo: les alentours de Lanzo, jusqu’à Ceres, 460-700 ${ }^{\mathrm{m}}$ (Stabile). Rare!

R. P. - Plaine du Po (nord): Vercelli, rives de la Sésia, $150^{\mathrm{m}}$ (Mella).

V. T. - V. de Varaita: Brossasco et autres endroits, $600^{\mathrm{m}}$ (IIortillet) - Val Scrivia: Serravalle près Novi (.Melia).

\section{Gen. HYALINA. (6)}

\author{
(IIyalina, Gray - in Turton: Shells. Bril.; 1840, mag. 168). \\ (Zonites (partım), Moquin-Tandon - Hist. Moll. France, 1855).
}

\section{Sect. C $\bigcirc \mathbf{U} \mathbf{U} \mathbf{S}$.}

(Comulus, Morquin-Tandon - in Mim. Acad. de Toulonse, 1848: IV, pag. 374).

\section{Hyalina fulva.}

Helix futera, Nüller - Verm. Hist. II. 1774; Drap. - Hist. Mull. 180 pl. VIl, fig. 12-13.

V. C. - Vallée de la Toce: Simplon, $2020^{\mathrm{m}}$, sous les pierres entassées, avec les Vitrines (Stabile) - V. de la Doire Baltea: collines de Viverone, $550^{\mathrm{m}}$ (Mella) - V. de la Stura de Lanzo: alpe de la Mussa, $1300-1700^{\mathrm{m}}$; sous les pierres (crystallines) près les ruisseaux, avec la Vitr. major, l'Ilelix glacialis, te Bul. quadridens, ete. (Stabile) - V. de la Doire Riparia: Nont-Cenis, près du lac, $1915^{\prime \prime \prime}$ (Mortillet); Monte Thabor, au-dessus de Rochemolles, 1500-1700m (Stabile).

R. P. - llaute vallée du Po: andessus de Crisolo (pied du Monte Viso) $1500-1800^{\mathrm{m}}$, sous les pierres rrvslallines (Stabile) - Plaine du 
Po (nord): Vercelli, $150^{\mathrm{m}}$ (Mella); rives du Tessin (Strobel); Turin (alluvions), $220^{\mathrm{m}}$ (Nortillet) - Plaine du Po (sud): Alexandrie, etc. (Strobel).

\section{Sect. EU I Y A I N}

(Euhyalina, Albers - in: Malakozool. Blätter, von Menke et Pfeiffer, 1857, pag. 91.)

\section{* Tyalina nitida.}

Helix nilida, Müller - Vermium Hist. II ; 1774, pag. 56.

- lucida, Drap. - Hist. Moll. 1805, pl. VIIl, fig. 11-12; (non Drap. - Tabl. Moll. 1801).

R. P. - Plaine du Po (nord): Vercelli, $180^{\mathrm{m}}$ (Mella) - Tessin; $80-100^{\mathrm{m}}$ (Strobel) - Plaine sud: Alexandrie (Strobel).

V. T. - V. Varaita: Brossasco, $600^{\mathrm{m}}$; sous les pierres des prés bumides (Nortillet).

\section{* 3. Hyalima Incida.}

Helix lucida, Drap. - Tabl. Moll. 1801; (non H. lucida, Drap. - Hist. Moll. 1805). - nitida, Drap. - Hist. Moll., 1805 ; pag. 117; pl. VIII, fig. 23.25.

Helicella Draparnaldi, Beck. - Index. Moll. etc. , 1837.

V. G. - V. de la Toce: alentours du lac Majeur, 190-500 (Stabile); Orta, $370^{\mathrm{m}}$ (Marani) - V. de la Doire Bàltea: Viverone, $550^{\mathrm{m}}$ (Mella) - V. de la Doire Riparia: Bussolino, environs de Susa, 400-500 (Mortillet).

R. P. - Plaine du Po (nord): rives du Tessin, 57-100 ${ }^{\mathrm{m}}$ (Strobel); Vercelli, $150^{\mathrm{m}}$ (Mella).

V. T. - V. de la Stura de Cuneo: Borgo-Sant-Dalmazzo près Cuneo, 450-500 (Mortillet) - Val-Tidone: Zavatarello (Strobel). 
5) Planulata, mihi. Tesha ulinque plumulatu-depressa, unbilico palubo.

V. C. - Cöles oceident. inférieures du lac Majeur (Belgirate, etc.) $190^{\mathrm{m}}$ (Stabile) - V. de la Doire Rip.: Chaumont, près Susa, (Mor(illel).

\section{* 1. Lyalina cellaria.}

Helix cellaria, Nuller - Ferm. IIist., 11, 1774; Mar. $2 \mathrm{~s}$

V. G. - Lac Majeur: Arona, 190-250"n (Prada) - Val Sesia: Oropaa, $450-500^{\mathrm{m}}$ (.)ella) - V. de la Doire Rip.: Bussolino, Chiomont au-dessus de Susa, $500-650^{\mathrm{m}}$ (Illortillet).

(3) Villae, Mortillet (llelix) (a) - in Strobel: Giormale di Malacologia, Anno 1. pag. 110; 1853. (Zuniles cugyrus, Stabile: Prospello Sistem. Slatist. Moll. di Lugano, 1859); matr. 51 - Helix Horlilleti, Stabile - in Pfeiffer L. : Monogr. Helic. viv.; IV. 1859; pag. 101.)

V. G. - Rives et fles du lac Majeur, 190-200m (Villa, Stabile, Mortillet.)

\section{* 5. Hyalina glabra. (b)}

Helix glabra, Studer - in Firussac: Tabl. System. 1822; Mor. Tandun - IIisl. Moll. de Frunce, 1855 ; pag. 80 , pl. IX, lig. 3-6.

V. C. - V. de la Toce: Côtes occidentales du lac Majeur, $200^{m_{3}}$ (Balsamo-Crivelli, Stabile) - V. de la Doire Bältea: Fontainemore, in Val-de-Lys, ou de Gressoney, $778^{\mathrm{m}}$ (Stabile) - V. de la Sura de

(a) Nun Ilelix Ville, Deshayes (in Firussac; tah). 90, ligr. 20).

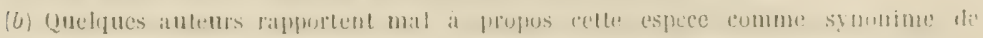

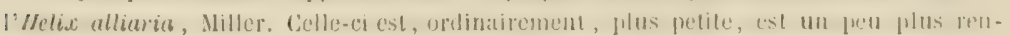

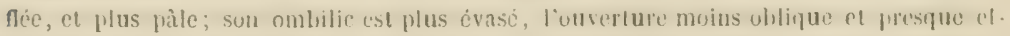

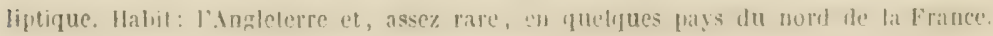


Lanzo: Ceres, Ala, Lanzo, Balme, 600-1400 $\mathrm{mm}$; sous les grusses pierren crystallines et serpentineuses; région des châtagniers, des sapins, mélèzes, etc. (Stabile) - V. de la Doire Ripaire: Suse, Chiomon?, Exilles, $: 00-800^{m}$ (Wortillet); plan-du-Col, dessus de Bardonnèche (région calcaire), $1300^{\text {m }}$ (Mortillet) - V. du Pellice: environs de Torrede Luserna (S. G. de Pinerolo), $\$ 30^{\mathrm{m}}$; sons les pierres de gneiss (Mortillet).

R. P. - Haute vallée du Po: Barge (entre la vallée du Po et celle du Pellice), 570-390 (Stabile) - Crisolo, 1380-1300m; ròches crystallines et serpentineuses (Stabile).

V. T. - V. Varaita: Venasea, b30-540m (Nortillet) Region gneissique.

Obs. Vit dans les bois, et dans les endroits humides, fraix, ombragés.

\section{* 6. Hyalina nitens. $(a)$}

Helix nitens, Gmelin - System. Natur., 1788 - Michaud: Complement a Draparnaus; 1831 ; pl. XV, fig. 1-3.

๔) Minnor. De la moitie plus petile que le type.

V. C. - V. De la Toce: Borca, Maccugnaga (Wonte-Rosa) sous les pierres crystallines, et l'écorce des arbres morts, parmi les débris pourris des vegetaux dans les endroits humides et ombragés; $1550^{\mathrm{m}}$ (Stabile) - V. de la Stura de Lanzo; au-dessus du village de Balme, etc. $1500-1800^{\mathrm{m}}$. Sous les grosses pierres (crystallines) près les neiges et les ruisseaux (Stabile) - V. de la Doire Riparia : Plan du Col au-dessus de Bardonnèche', $1300^{\mathrm{m}}$ (Mortillet) Rég. calcaire.

(a) La veritable Hyalina nilidula (Helix), Drap. parait ne pas exister en Piémont, ni en Lombardie, ou bien y être éxtremement rare; la forme petile de la Hyal. witens (Helix), Gmelin et Michaud, est presque toujours confondue avec la Hyal. milidula. Cette derniere est assez rare, meme de l'autre côté des Alpes. On pourra riconnailré la IIyal. nitens, quoique en exemplaires assez petits, à son ombilic lar'ge, à sun dernier tour fort-dilaté vers la butuche, et obliquement deflechi en forno de tuit un pou deseendant vers la suture, a sa bouche ovale, clliptique. tris oblingte. 


\section{* Jyalina hiulea}

Mrelie hiulca, dill.

- cellaria, Muller: Var. a, Porro - Malacol. Comasca, 1838: pl. 1, fig. 1.

V. C. Alentours du Verbano, ou lac Majeur, 200-500 (Mousson, Stabile).

8. Hyalina Petronellae. $(a)$

Iletix vitrina, Fúrussac - Tablean System. 1822, pag. 45; (non H. vitrina, Spis).

- Petronella, Charpentier - in Mortillet et bumont: Mist. Mollusq. de Sazoje; 1853 , pag. 230; P'teiffer L. - Monogr. Helic. vic. Vul. IlI, 1853.

V. C. - V. de la Doire Bàltea: l'Allée-Blanche, près des chàlets qui font face au glacier du Viage, $2100^{\mathrm{m}}$ (Mortillet) - Val-de-Lys, ou de Gressoney: Gressoney-la-Trinité, sous les pierres (gneiss), $1700^{\mathrm{m}}$ (Stabile) - V. de la Stura de Lanzo: village de Balme, $1500-1800^{\mathrm{m}}$ (Stabile) près des neiges ou des ruisseaux; assez rare partout, sous les gros blocs de gneiss, avec la II. fulva, l'Ilelix glacialis, etc.

\section{*. Hyalina hydatina.}

IIelix hydalina, hossmïssler - Iconogr, d. Land-und Süssu; ; VI, VIII, fig. 5פ9; 1838.

R. P. - Plaine du Po (sud): Stradella (Strobel). M. ${ }^{r}$ Strobel dit d'avoir trouvé cette espèce même dans les alentours de Pavie (!). La limite inférieure de la plaine cispadane n'est pas loin d'ici!.

V. T. - Val Tidone: Zavatarello (Strobel).

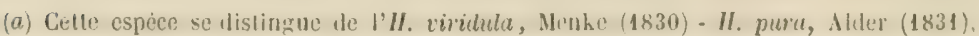
par ses dimensions majeures, par ses lries plus rigulieres el prononcées, par sun lecrier four el sa houche arrondis. 


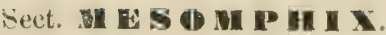

(Hesomphix, Rafinesque - in Beck: Index Holluse. 1837.)

\section{Hyalina olivetorum.}

ẼTelix olivelonm, Hermann - ex Schrüter: Einleitung in die Conchylienkernnluis nach Linné; II, 1784.

- - Gmelin - System. Natur., 1788. (Non Hel. olivet. Fèr., Lamk, Russm.) - Leopoldiana, Charpentier (in sehedis).

Hyalina (Mesomphix) Leopoldiana, Albers - in: Malak, Blatter; 1857.

R. P. - Collines au sud du Pô: environs de Rosignano, San. cluaire de Créa, 200-400 (Mella) - Montalto, à la droite du Tánaro; Torrazzelta, en Val Coppa (Strobel) - V. de la Bòrmida (Doria).

V. T. - V. de la Scrivia: Serravalle près Novi (Mella); Arquata (Villa) - V. Tidone: Monte Penice; - V. Staffora (Strobel).

Obs. Vit dans les lieux fraix, souvent parmi les buissons et entre les racines; s'enfoncant plus ou moins sous le gazon, et même dans la terre; ne se montre que par les temps très-humides; et on la trouve, généralement, adulte vers l'automne (Mortillet).

\section{Gen. HELIX.}

(Helix (emendat.), Linne - System. Natu. edit. X, I; 1738. )

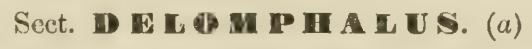

Delomplalus, Agassiz - in Charnentier: Calal. Holl. de la Suisse, 1837. - Palule

(partim), Helıl - in: Isis; 1837, pag. 916.)

\section{* 1. Melix pyganaea.}

Helix pygmaea, Draparnauil - Tabl. des Moll., 1801; et Hist. Holl. 180j; pl. VIII, fig. 8-10; Moguin-Tandon - Hist. Moll. de France; 1855; pag. 103, (animal).

V. C. - Vallée de la Toce: Col dı Simplon, sous les pierres (gneiss) dans les endroits humides, marécageux, $201 \mathrm{5}^{\mathrm{m}}$ (Stabile) -

(a) Les especes de cette section ressemblent le plus alux Inyalines (Zonites, partim). Màchoire à còtes nombreuses, maí assez minces, et à lurd inférieur crénclé. Vésicules mug̣ueuses nulles, ni puche a clasil. 
V. de la Doire Billea: Gressoney-la-Trinite (Val de Lys on Gressoney\}, $1670^{\mathrm{mz}}$ (Stabile) (région gneissique).

R. P. - Alentuurs du Tessin, près de Pavie, etc. 37-80 (Del Mayno) - Vercelli, $150^{\mathrm{m}}$ (Nella).

\section{æ. Delix ruderata.}

Helix muderule, studer - Kurze's lerzeichniss der bis jelz in unserm Vaterlande entdecklen Conchylien; in Gïrtner: Naturwis. Anzeig. Schueiz. Gesellschaft; Bern, 1820; - Hartmann: in Neue Alpina, 1821; Jl. H, Ifg. 11; - Muquist-Tandon: loco citato, pag. 105 (animal).

V. C. - V. de la Doire Bàltea: Gressoney-la Trinité, $1670^{\mathrm{m}}$ (Stabile) - l'Allće-Blanche, vis-à-vis le glacier du Miàge, $1700^{\mathrm{m}}$ (Dumont) - V. de la Stura de Lanzo: au-dessus du village de Balıne, Alpe de la Mussa (avec la Hyalina fulva, Hy. Petronelle, etc.) sous les grosses pierres (crystallines), 1300-1700" (Stabile) - V. de la Doire Ripaire: Mont-Cénis, $1913^{\mathrm{m}}$ (Mortillet); Oulx (route du MontGenève), $1060^{\mathrm{m}}$ (Stabile). Dans les régions moins élevées vit sous l'écorce des arbres morts, et parmi le détritus végétal; lieux boisés (Aunes), Lumides. Terrains crystallins en genre.

\section{* 3. Helix not undata.}

Hetix votundatu, Muller - Vermiun Hist.; 11, 1776; - Iraphirnand: Hist. .Holl. . 1805 , pl. VIII, tig. 4-7; Joq.-Tamion - Moll. de France, 1855, p. 107, H. X, fig. 9-12 (Anatom.)

V. C. - V. de la Toce: Domo d'Ussola $500^{\mathrm{m}}$ (Strobel); rives du Verbano, 200-300 (Stabile): Orta, 370-400 $^{\mathrm{m}}$ (.Varani) - V. de la Doire Bälea: Viverone, $580^{\mathrm{m}}$ (Mella) - V. de la Stura de Lanzo (Ala et Gros-Cavallo): Céres, $710^{\mathrm{m}}$; Al-Forno, 1250-1250m (Stabile) - V. de la Doire Rip.: Chiomont, Suse, 500-650m (Strobel, Mort.). R. P. - Haute vallée du Pó: au-dessus de Crisolo, 1380-1400 (Stabile); plaine du Pô (nord): rives dı Tessin (Strobel); Vercelli, $180^{\mathrm{m}}$ (Mella).

V. T. - Apennins piémontais (Strobel). 


\section{*. Helix rupestris.}

Helix rapesiris, Draparnaud - Tabl. Moll., 1801.

a). rupieala, mihi - Prosp. Sist. Stat. Molt. Lugano, 1859 - Testa elatitiscula, umbilico angustiori.

B). saxatills, Hartmann - System. Gasteropod. pag. 52, $1821=$ Ilel. spirtula, Villa: Disposit. system. Conch. 1811. - Testa depressiuscula, umbilico latiori.

V. C. - V. de la Toce: Domo d'Ossola, $300^{\mathrm{m}}$ (Prada); alentours du Verbano, 194-1000 (Stabile) - V. de la Doire Bảltea: l'AlléeBlanche, $1700^{\mathrm{m}}$; Vallée d'Aosta, 600-1800 (Francois Dumont); Courmayeur, $1200^{\mathrm{m}}$ (Bellardi) - V. de la Stura de Lanzo: la haute vallée, $1300-1600^{\mathrm{m}}$ (Stabile) - V. de la Doire Riparia : Suse (Mortillet); MontCénis (Strobel); au-dessus de Rochemolles au pied du Monte-Thabor, 1650-1700m (Stabile); Pas-des-Echèlles, 1790 ${ }^{\mathrm{m}}$ (Strobel). Dans les régions moins élevées vil sur les rochers (calcaires et crystallins), parmi les mousses, etc. Dans les régions élevées on la rencontre sous les grosses pierres (gneiss) avec le Bul. quadridens (minor), l'Helix glacialis, etc. La mutation $\alpha$, je l'ai trouvée typique sur les rochers au-dessus de Rochemolles.

Sect. I. U C N A. (a)

(Liecena, Ilartmann - System. Gasteropod.; 1821, pag. 40; won Oken)

\section{* 5. Helix pulchella.}

Slelix pulchella, Draparnaud - Tabl. des Mollusq.; 1801, pag. 90: Moquin-Tandon - Moll. de France, 1855; pag. 140, pl. XI, fig. 28-30 (Anatom.)

a) Inornata , mihi - Prospetto Sistere. Slatist. Moll. di Lugano, 1859; H. pulchella, Var. $\beta$ Drap. - Hist. Moll. 1805 ; pl. VII, fig. 33-34.

V. C. - Val-Sésia (V. del Cervo): Biella, $480^{\mathrm{m}}$ (Cesati) - V. de la Stura de Lanzo: Balme, $1500^{\mathrm{m}}$; sous les pierres dans les endroits marécageux (Stabile).

(a) Michoire à còtes tres-nombreuses, serrées, mais jeu prononcies; bord inférieur is peine crénølé. Vésicuics murqueuses (dans cette esprece) nulles. 
R. P. - llanfe vallée du Po: au-dessus du village de Crisolo, $1400^{\mathrm{m}}$ (Slabile). Plaine du Pú (nord): Vercelli, $180^{\mathrm{m}}$ (Mella); Turin, $200^{\mathrm{m}}$ (Mortillet); Mez:ana-Corti, prés La-Cava (Strobel) - Plaine sud: Alexandrie, $90^{\mathrm{m}}$ (Mortillet): Tortone, sur les coleaux (Mortillet).

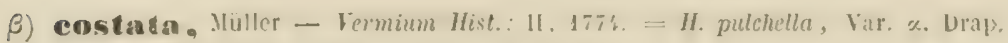
Hist. 11)ll. , 1805: pl. 17l . Mr. 3032.

V. T. - Vill-de la Treblua (Val-Tidone): Zavalarello (Strobel).

\author{
sect. 'T
}

(Trigmostoma, Fitzinger - System. Verzeichn.; 1833.

*. Melíx angignger. (b)

Helix angigyia, Ziegler - in Rossmüssler: Iconogr. d. Land. und Sïssu. Moll., 1835, I, pat. 70,10 . $21 \%$.

V. C. - V. le lat Doire Riparia: Suse, \$00-600 (Mortillet).

C'est une chose à remarquer que celte espèce n'ait été pas trouvec

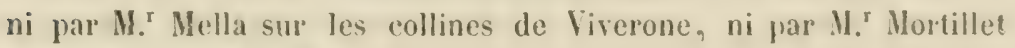
sur les collines de Turin!

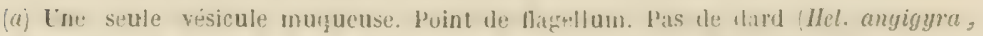

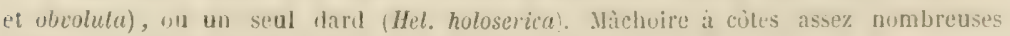
$(12-14)$, un jue serrées, saillantes, et a lworl crincle.

(b) M. ${ }^{r}$ Alfred de Saint-Simon est le premier gui ait fait de diligentes observations sur celle espece, de mème que sur l'Hel. naulilifurmis, et l'Hel. cingulata, Var. Luganensis, ete. des quelles je lui remettais, en $48 \%$, quelques exemplaires vivants et licen cunservis. Il a runstate mime sur f'Hel. angigyra une mìchoire ayant de 12 a 16 stries verticales, paralleles, pru distincles, repondant a autant de dentieules a peine frononeres; une seule visicule muqueuse, et l'absence du flagellum el de la poche à dard. (Saint-Simon: Niscellunées malacol.; Dieade 1. 1848; et 11, 1836. Tuuluuse). Voir aussi mon: Prospello Sistem. Stut. dei Mollusehi di Lugano; in Atti Sue. ital. se. mat. 185! : Milinu. 


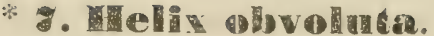

Jelix obvolula, Nuller - Verm. Hist. 11, 1774; Druparnaud - Hist. Moll. 1805, pl. VII, fig. 27-29.

- holosericea, fimelin - System. Nalur. 1788; non Studer; Moq.-Tandon - Moll. de France, 1855; jag. 114, pl. X, fig. 26-30 (Anatom.)

V. C. - V. de la Toce: Orta, $370^{\mathrm{m}}$ (Marani); Domo d'Ossola, $506^{\mathrm{m}}$ (Prada); toutes les rives du Verbano, 240-300 ${ }^{\mathrm{m}}$ (Stabile, Prada) - V. de la Doire Bàltea: Fontainemore en Val-de-Gressoney, ou Val-de-Lys, $780^{\mathrm{m}}$ (Stabile); alentours de Viverone près de Ivréa, $550^{\mathrm{m}}$ (Mella) - Val de la Stura de Lanzo: Ala, Céres, 700-1080 (Stabile) - V. de la Doire Riparia: Chiomont, Suse, 500-650 (Mort., Strobet).

R. P. - Haute vallée du Pô: Barge, $380^{\mathrm{m}}$ (Stabile) - Plaine du Pô (nord), Turin, rio de la Battería, 250 (Nortillet, Mella).

V. T. - Val Maira: Dronéro, $600^{\text {ni }}$ (Mella); - Val Stura de Cnneo: Roccavione entre Cuneo et Limone, $800^{\mathrm{m}}$ (Mortillet) - Val-Stafffora et Vallée du Tidone (Nousson, Strobel) - Nonferrat (Rezia).

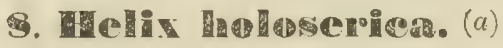

Jelix holoscricea, Sluder - hurz. Trrzeichniss.; 1820, pag. 87; non Gmelin.

- holoserira, Rossmïssler - Land. und Süssw. Moll. I, 1835; fig. 20.

V. C. - V. de la Doire Bàltea: Ilaute vallée d'Aosta (Nortillel). On peut dire que cette espèce est à peine représentée dans la faune ản Piénont par quelques rares individus qui ont franchi, pour ainsi dire, les sommets alpins, et de la Savoje sont passés sur le versant italien mais ne descendant pas au-dessous des régions élevées. Cette jolie espèce dans la vallée supérieure du Tessin, ou Lepontine, (versant méridional du S. Guthard) habite, quoique assez rare, les forèls au dessus de Airolo, et mème celles du Dazio-Grande (1000-1400 $\left.\mathrm{m}^{\mathrm{m}}\right)$ : dans la partie supérieure de la Valle-Maggia, je l'ai récoltée, à Fusio $\left(1280^{\mathrm{m}}\right)$ parmi les gazons, pendant une grande pluie, en juin.

(a) Il faut écrire holoserica, el non holosericen. 


\section{Sect. D I E A A O S T O M A. (6)}

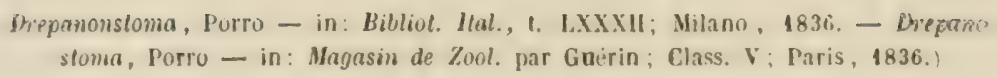

\section{*. Helix nantiliformis.}

Drepanonsloma (subgen. Ilelicocycla) nautiliformis Porro - in: Bibl. Ital. (loe cit.;

Drepanostoma nautiliformis, Porro - in: Magasin. Zool, ete. (loco cit.); et in: Molacologia Conasea; 1838, pag. 23, pl. I, fig. 3.

V. C. - Val de la Toce (Val-Anzasca): Calasca, Maecugnaga, $600-1400^{\mathrm{m}}$; assez rare, dans ces localités, sous les pierres crystal. lines dans les endroits ombragés et boisés (Stabile) - Alentours du lac Majeur: Stresa, Belgirate, $200^{\mathrm{m}}$ roches micaschisteuses (Stabile). Val-Sésia: Varallo, 400-600m (Nella) - V. de la Doire Bàltea: collines de Viverone (N-E de lvréa), $530^{\mathrm{m}}$ (Mella) $-\mathrm{V}$. de la Stura de Lanzo: région inférieure de la vallée, $480-71 \mathrm{~B}^{\mathrm{m}}$ terrain micaschisteux, crystallin et métamorphique en genre (Stabile).

\section{Sect. A C A N T II I U I A}

(Acanthimula, Beck - Berichl der Naturforsch. in Kiel; 1846.)

\section{Helix aculeata.}

Helix aculeata, Müller - Verm. Hist.; II, 1774.

Trochilus terrestris, Da Costí - Test. Brit. 1778; pag. 166, pl. II, fig. 1-5; Draparnaud - Mist. Moll.; 1805, pl. VIl , fig. 10-11: Moquin-Tandon - Moll. de France, 1855 ; pag. 190 (animal).

V. C. - V. de la Doire Bàltea : collines de Viverone près Ivréa, $350^{\mathrm{m}}$; avec l'H. nautiliformis; très-rare (Mella).

R. P. - Vallée du Ticino (Del Mayno).

(a) Mâchoire arquée, peu rohuste, à 8 cútes verticales, parallèles, sinneuses, peu saillantes, qui correspondent à un égal nombre de denticules tris-imoussées. Une seule vésicule muqueuse; point de fagellum, ni de poche à dard. (Saint-Simun: loc. cit.) 


\section{$-59-$}

Obs. Espèce très-rare partont, quoique repandue en toute Europe, de la Suède jusqu'en Sicile, en Angleterre, en Portugal, dans les îles Acores! - La Russie, la Grèce et la Turchie seraient les seuls pays où cette charmante espèce n'aurait été pas encore trouvée. La monographie de l'H. aculeata (a) par M. ${ }^{\mathrm{r}}$ Henri Drouët, l'un des savants naturalistes contemporains, est très-intéressante, et on peut dire, sans offenser la modestie de l'auteur, que ce petit travail est un chef-d'œuvre d'exercice monographique.

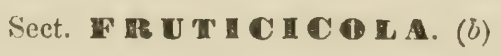

(Fruticicola, Ileld - in: Isis; 1837, pag. 914.)

† Mâchoire à côtes nombreuses (14.20), peu apparentes; denticules marginales peu sensibles

a) Eradybaena (partim), Beck - Ind. Moll. 1837, pag. 18.

\section{* 1. Melix cilliata}

Ifelix cilialu, Venetz; in Studer - Kurz. Verzeichn.; 1820, pag. 86. - Rossmässler : Iconogr. d. Land. und Süssio. Moll. VIII, 1838; pl. 31, fig. 430; MoquinTandon - Moll. de France, 1855; pag. 217, pl. XVII, fig. 1.5 (Anatom.)

V. C. - V. de la Toce: Baveno, $200^{\mathrm{m}}$ (Mousson) - V. de la Doire Riparia: Oulx (route du M. Genève) $1070^{\mathrm{m}}$; sous les pierres avec l'Hel. hispida, dans les endroits ombragés et humides; rare et de petile taille.

R. P. - Plaine du Pó (nord): Turin, rio della Batteria $280^{\mathrm{m}}$

(a) H. Drouët : Exercice monographique sur l'Hel. aculeata - in: Annales de la Soc. Linn. de Maine et Loire, III vol. 1859.

(b) Mâchoire à côtes nombreuses (jusqu'à 20), et à marge denticulée. Vésicules muqueuses rarement nulles, ordinairement au nombre de 2, divisées en deux à cinq branches. Dard nul (Hel. ciliata, strigellı, carthusiana, et Galloprovincialis); 1 dard (Hel. fruticum, cinctella, incarnata, sericea); 2 dards (Hel. hispida). 
(Mortillet, Cesati) - Plaine sud: Montalto, ete. Collines entre la Pál Aversa el la Val-Coppa ì la droite du Tanaro.

V. T. - V. de la Trebbia (Val-Tidone): Zavatarello (Vousson).

\section{Melix sericea.}

Ifelix sericen, Draparmaul - Tablean des Mall. 1801: cl Hist. M:3l. 1803; pi. VII, fig. 16-17; non II. sericea, Nuller.

V. C. - V. Doire Riparia: environs de Suse, $810^{\mathrm{m}}$ (Mortillet).

V. T. - Val-Maira (région gneissique): Dronéro, $600^{\mathrm{m}}$ (.Mella).

\section{* 13. Melix Inispida.}

Jelix hispida, Linne - System. Nalur., Edit. X, 1758, 1, pag. 771.

Bradybema hispida, Beck - Ind. Moll., 1837; Moquin-Tandon - Moll. de France, 1855 , pag. 22' (animall.

V. C. - V. de la Doire Ballea: Courmayeur, $1218^{\mathrm{m}}$ (Bellardi). V. de Lys, ou de Gressoney, $700-1400^{\mathrm{m}}$ (Stabile) - V. de la Stura de Lanzo: toute la vallée, de Balme à Céres, 700-1500 herbes dans les prés, parmi les débris des végétaux, etc.; après les pluies sur les sentiers montagneux qui longent les paturàges on peut aisement s'en procurer, car il monte sur les tiges et les sommets des herbes les plus grandes (Stabile) - V. de la Doire Riparia: MontCénis, à la Grande-Croix $1900^{\mathrm{m}}$ (Mortillet). Dans toutes les localités indiquées, le sol est de formation crystalline (gneissique); route du Mont-Genève: Oulx (endroits fiaix, ombragés, près les ruisseaux), $1070^{\mathrm{m}}$ (Slabile).

R P. - Plaine du Pó (nord): Turin, 207 (Mortillet, Cesati)

- Plaine sud: Alexandrie, $90^{\mathrm{m}}$ (Strobel): Asti (collines tertiaires), $110^{\mathrm{m}}$ (Mella).

V. T. - Vai Vataita: prairies de Brossaseo, 600-700m (.Mort.). 
1) Welicella (partim), Férussac - Tabl. System., 1822.

\section{* 14. Melix strigella.}

Helix strigella, Drap. - Tubl. Moll. 1801; et Hist. Moll.; 1805, pl. VII, fig. 1.2; Moquin-Tandon - Moll. de France, 1855 ; pag. 204 (animal).

V. C. - V. de la Toce: Calasca (Val-Anzasca), 500-600 ${ }^{\mathrm{m}}$ (Stabile); rives du Verbano, 200-300 ${ }^{\mathrm{m}}$ (Stabile, Prada) - Val-Sésia (V. del Cervo): Biella, 480 (Mella) - V. de la Doire Bàltea: collines de Viverone, près de Ivréa, $\mathbf{5 3 0} 0^{\mathrm{m}}$ (Mella).

R. P. - Haute vallée du Pô: Paesana, Crisolo, 340-1380m (Stabile) - Plaine du Pô (nord): Vallée du Ticino, Novara (Strobel); Vercelli, Turin, 180-200m (Cesati, Mortillet) - Plaine du Pô (sud): Alexandrie $90^{\mathrm{m}}$; Montalto, à la droite du Tanaro (Strobel).

V. T. - La Torre près Pinerolo, (Val Pèllice), B30 ${ }^{\mathrm{m}}$ (Mort.).

Obs. Vit dans les bois, sur le coteaux, au milieu des haies et des buissons, le long des murs, rarement sous les pierres.

Lorsque la coquille est fraiche et contient l'animal vivant, est couverte d'un duvet assez épais et visible même à l'œil nu. Le dimensions de cette espèce varient beaucoup: (Lat. 10-11 $1 / 2$; Alt. 6-6 $1 / 2$. millim.; exemplaires recueillis à Lugano; Lat. 12; Alt. 8 millin.; Anfract. 5. exempl. de Bẻx, Suisse occidentale; Lat. $151 / 2$; Alt. 9 1/2; Anfract. $81 / 2$. Nilano, Piémont, Russie, etc.). Il parait cependant que les individus vivants sur les hauteu's n'atteignent pas les grandes dimensions de ses confrères qui habitent à la plaine, et spécialement près les vieux murs dans les endroits humides, sauvages, et au milieu des haies, etc. L'Hel. hexagyra, Megerle n'est autre chose que la forme plus grande de l'Helix strigella; mais comme il y a de formes intermédiaires, ainsi le nom imposé par Mlegerle ne peut avoir d'autre valeur que celle d'une simple mutation! 
c) Wonacha, Fitzinger - System. Terzeichn., 1833.

\section{* 15. Helix incarnata.}

Helix incurnata, Muller - Verm. Hist.; 11, 1774, pag. 63; Draparnaud - Hist. Moll. 1805 , fil. VI, fig. 30.

V. C. - V. de la Doire Bàltea (V. de Lys, ou de Gressoney): Fontainemore, $778^{\mathrm{m}}$ (Stabile) - V. del Cervo: Biella, $480^{\mathrm{ml}}$ (Cesati).

R. P. - Plaine du Pô (nord): Vercelli, $1 \$ 0^{\mathrm{m}}$ (Mella).

B) Armata, mihi - Prospelto Sistem. Stat. Moll. di Lugano, 1859. = Itelix monodon, Villa; non Fúrussac - Margine columellari peristomalis twberculum callosum ferente.

V. C. - V. de la Toce: rives du Verbano, 210-250m (Stabile) V. Sésia (V. del Cervo): Biella, 450 (Cesati). Dans toutes les localités citées ci-dessus cette espèce vit sous les feuilles pourries, entassées, on sous les grosses pierres, les morceaux de bois pourri, dans les licux humides, sylvestres. Rare partout.

Obs. La callosité du bord columellaire n'est pas un caractère suffisant pour constituer une division spécifique; ces pièces accessoires chez cerlaines espéces dépendent, en général, d'une surexcitation de vitalité dans le mollusque, due à des conditions spéciales dans lesquelles le mollusque vit. Cependant je ferai observer que la mutation armata paraît être bornée au sud des Alpes; de l'autre côté de la chaine alpine l'Helix incarnatı est d'une taille plus grande, plus forte, sans callosité, et à ombilic plus ouvert. (Hel. incarnata, Müller; Var. silvestris (Monacha), Hartmann). Dans nos pays la taille est moyenne, et quelquefois même assez petite. Ces deux formes, ainsi que les deux mutations, avec et sans callosité, sont melées souvent dans une même localité. 
d) Temolola, Gray - Nat. arrang. Moll. in Med. repos. XV, 1821, pag. 239.

\section{Helix cinctella.}

Helix cinctella, Draparnaud - Tableau des Moll. 1801 ; et Hist. Moll. 1805, pl. VI, fig. 28; Moquin-Tandon - Moll. terr. et fluv. de France, 1855; p. 215 (animal).

R. P. - Plaine du Pô (sud): Valmadonna, Alexandrie, Montalto, Voghera, Cicognola, Stradella, $50-100^{\mathrm{m}}$ (Strobel).

V. T. - Valcoppa; Val Aversa; Val Tidone: Romagnese; Val Trebbia: Bobbio (Strobel) - Corniasca sur les coteaux de la rive droite de la Scrivia (Mortillet).

e) Theba (nartim), Risso - Hist. nat. Europ. mérid., 1826; IV, pag. 73.

\section{* 1\%. Melix carthusiana.}

Helix carthusiana, Nüller - Vermium Hist., II, 1775; non Drap.

- carthusianella, Draparnaud - Tabl. Moll. 1801; et Hist. Moll. 1805; Rossmäs. sler - Icon. Land. und Süssı. Moll. 1837, VI, p!. 27, f. 366, c. d.

V. C. - Vallée de la Doire Bàltea: collines de Viverone, $330^{\mathrm{m}}$ (Mella) - V. de la Stura de Lanzo (Stabile). Terrain crystallin et alluvionaire.

R. P. - Plaine du Pó (nord): Novara, Vercelli, Mezzana Corti, toute la Lomellina, Turin, 90-260 ${ }^{\mathrm{m}}$ (Mella, Mortillet, Strobel) - Plaine sud: Alexandrie, Valmadonna, Montalto, Valenza, Astésan, Voghera, Stradella, 80-1 $80^{\mathrm{m}}$ (Strobel).

V. T. - V. du Tanaro (Strobel) - V. de la Bormida: Acqui (Villa, Stabile, Bonomi) - Val Scrivia: Tortona (Mortillet) - Val Staffora; Val Coppa; Val Aversa; Val Tidone: Zavatarello (Strobel).

B) Ieveoloma, mihi - Prosp. Sist. Stat. Moll. di Lugano, 1859 - Testa minore, peristomate omnino albo.

V. T. - V. de la Bormida : Acqui (Stabile, Bonomi). 
Helix cathasiana, Draparmaud - Tabl. Moll., 1801; non Miller.

Theba carthusiana, Risso - Hist. nat. Europ. mirid. 1826. IV. pag. 74.

Nelix Cantiana (partim), Mntagu. - Test. Brit. 1833, pag. 422.

- Galloprovincialis, Dupuy - Hist. Moll. terr. el deau douce de France, 18:8, Janvier; pl. IX, lig. 5, c. : Moguin-Tamdun - Hist. nat. Moll. de France; 1855, pag. 202, pl. XVI, fig. 13; Alulphe Sehmidl - der Geschlechlsapparat der Stylommatophoren, etc. Berlin, 1855; pl. VII, fig. 52. (Anat.

V. C. - V. de la Toce: rives du Lac Majeur (Strobel) - V. de la Doire Riparia: Suse (Strobel).

R. P. - Plaine du Pó (nord): Turin, 220-250 (Hortillet) - Plaine sud: Broni, Stradella (Villa).

V. T. - V. Stura de Cuneo: Vignolo près Cuneo (Mortillet) - V. de la Bormida: Acqui (Villa, Stabile, Bonomi) - Val-Staflóra; Val-Coppa; Val-Scurpasso; Val-Aversa; Val-Tidone; Monte-Pénice; Bobbio en Val-Trebbia (Strobel).

Obs. La véritable Melix Cantiana Montagu, de l'Angletere et de la France septentrionale, n'existe pas en Lombardie, ni en Piémont: mais elle y est rémplacée par l'Ilelix Galloprovincialis iII. carthusiana, Drap.; non Mäller), un peu plus petite, à dernier tour moins deprimé, à couleur un peu plus claire, à bouche plus arrondie, à ombilic plus étroit, à péristome blane en dedans, roussattre en delsors; quelquefois celle conleur s'élende mème sur une portion du dernier tour. Les divers individus de celle espece récoltés par moi, Strobel, Villa, etc. en Piémont, varient de 8,5 a 11,5 mill. de haut.; et de 11,5 a 16,5 mill. de diam. A peu près les mèmes dimensions présentent les individus que j'ai trouvé en toute la Val-d'Olona daus le Milanais. Les quelques spécimens, que je dois à la bonté de II. Issel, des environs de Gènes, ne sont guère plus grands; mais dans le Véronais l'Helix Galloprovincialis atteint des proprortions considerables! Les exemplaires que j'ai reçus de .V. ${ }^{r}$ le doet. Martinali de Vérone et des

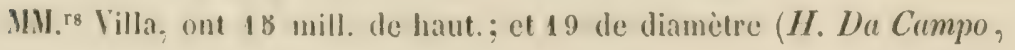


Villa). Les exemplaires de cette localité ne présentent pas la zone d"ur? corné plus pâle au dernier tour, comme on la voit quelquefois sur les individus de la Val-d'Olona.

† Mâchoire à côtes peu nombreuses (5-9), saillantes, un peu espacées; denticules marginales aiguës, inégales.

i) Ealota, Hartmann - Erd-und Süsswass. Gus(eropod.; 1, 1840.

\section{* 19. Helix fruticum.}

Helix fruticum, Müler - Verm. Hist. II; 1774, pag. 71; Draparnaud - Ilist. Hull. 1803 , pl. V, fig. 16.

Eulota fruticum, Hartmann - loco citato; Moquin-Tandon - Moll. de France, p. 197, fl. XVl, fig. 1-2 (Anatom.)

R. P. - Plaine du Pô (nord): Plaine du Tessin (Strobel) - Plaine sud: Collines d'Asti, $140^{\mathrm{m}}$; Sanctuaire de Créa (au sud de Casale), $440^{\mathrm{m}}$; Dronéro (à l'entrée de la Val.Maira), $600^{\mathrm{m}}$ (Mella).

Sect. X E I I P II工A. (a)

(Xerophila, Held - in : Isis, 1837; pag. 913.)

\section{* 20. Helix cespitum.}

Helix cespilum Draparnaud - Tabl. des Noll. 1881; et Hist. Holl. 1805; 1]. VI, fig. 14-15.

a) introdmeta, Ziegler - Testa unicolore, sæpe 1, vel pluribus angustis fasciis ornata; umbilico latiori. (Pour l'anatomie, voyez: Ad. Schmidt: Geschi. d. Stylommatoph. 18305 ; pag. $31, \mathrm{pl}$. VII, fig. 42.)

R. P. Plaine sud du Pô: Alexandrie, 82-90 ${ }^{\mathrm{m}}$ (Marani); Voghera (Strobel).

(a) Mâchoire à côtus ( $\left.\begin{array}{lll}5 & \text { a } & 10\end{array}\right)$ plus ou moins saillantes et icartées, et a bord denticulé ou crénelé. Vésicules muqueuses nombreuses (8 à 25). Dard \& ou 2 . Flagrellum rourt, subulé. 
V. T. Vallée de la Stura de Cuneo (Val Vermegnana): Borgo S. Dalmazzo, Limone, route du col de Tende, 600-1018 ${ }^{\mathrm{m}}$ (Mortitlet) - Val de la Bormida: Aqui (Villa, Bonomi) - Val de la Scrivia: Tortone (coteaux); Serravalle, Busalla (chemin de fer de Gênes) (Mortillet, Mella) - Val Staffora; Val Trebbia: Bobbio (Strobel).

\section{* 21. Helix Ammonis.}

Helix ericetorum, Collect. et Catalog. Lombard.; non Muller.

- candicans (partim), L. Pfeiffer - Monogr. Ileliceov. viv.; 1, 1848, jag. 164.

- candicans (partim), De Bella et Martinati - Cat. Moll. Venet. 1855; jag. 3 i pl. 1, fig. 1-8.

- neglecta Var., Strubel - olim.

- Ammonis, Adolphe Schmidt - in litt.; Strobel - Essai d'une distr. orogr.géogr. Moll. terr. Lombard. éte. in: Mém. Acad. Sc. de Turin, Sèr. II, tum. XVIII ; 1857 .

R. P. - Plaine du Pô (nord): Vallée du Tícino (Strobel) - Plaine sud: Valenza, Alexandrie, Voghera, Casteggio, Montebello, Stradella, Cicognola (Strobel, Villa).

V. T. - V. de la Scrivia: Tortone (plaine et côteau); Serravalle, Arquata (Mortillet) - Val-Staffora (plaine et côteau); Bobbio en Val Trebbia (Strobel).

Obs. Cette espèce, de mème que la précédente, vit dans les endroits secs le long des chemins, sur les bords des champs, sur les arbustes et les pelouses. La spire est, en général, un peu plus élevée que dans les exemplaires de Milan et de Vérone. L' $\boldsymbol{H}$. Ammonis ressemble à l'H. ericetorum, Müller, mais on la distinguera facilement à sa spire un peu plus élevée, au dernier tour moins étroit, et d'oủ il en résulte un ombilic moins évasé et plus étroit que celui de l' $H$. ericetorum; à son ouverture plus arrondie et à bourrelet assez fort, roussâtre ou violâtre, très-rarement blanchàtre. L'II. Ammonis a beaucoup de ressemblance avec l' $H$. candidu, Porro (candicans (partim), Ziegler, in Pfr. loco citato), mais cette dernière a le test plus blanc, les bandes rares et ćcartées, plus souvent unirolore, c'est à dire tout blanc; l'ombilic est plus étroit, l'ouverture 
plus oblique et à bourrelet d'un blane laiteux. La veritable $\boldsymbol{H}$. erícetorum habite de l'autre côté des Alpes (France, Allemagne, Suisse). J'ai trouvé, cependant, pour la première fois cette espèce, assez abbondante du côté sud des Alpes Suisses, entre "il Dazio " et Airolo (route du S. Gothard) sur les murs à sec, et les grosses pierres gneissiques qui flanquent les prés sur la grande route (à $\left.980^{\mathrm{m}}\right)$; ces exemplaires sont un peu petits, et la forme et la couleur du test et des bandes rappellent plutôt celles de l'H. Ammonis, mais l'ombilic est bien celui de l' $H$. ericetorum, et le bourrelet est blanchâtre. L'H. Ammonis est le représentant de l'ericetorum en Lombardie, dans une grande partie du Vénitien, et en Piémont. L'H. candidas Porro, habite le Tyrol italien (Stabile), la plaine et les collines de Mantoue et de Brescia (Strobel, Spinelli) avec l'H. Ammonis, mais elle n'y est pas trop commune.

\section{Helix profuga.}

Helix striata (partím), Draparnaud - Hist. Moll.; 1805.

- caperata (partim), I. Pfr. - Monogr. Helic. viv. I, 1848, pag. 167.

- profuga, Ad. Schmidt - in: Malak. Bläter; 1854, pag. 18; et Stylommat, etc. 1855; pag. 30 , pl. VI, fig. 38 . (Anatom.)

V. T. - Vallée de la Scrivia: Tortone (côteau); Serravalle, Arquata (Mortillet).

Obs. Cette espece est très-commune à Milan; à la plaine et sur les collines: Côme, Brescia, Mantoue (Spinelli, Strobel), Venise (Mortillet). On la rencontre jusqu'à Florence, etc. Une varieté blanche n'est pas rare à Trieste. Dans le Napolitain et en Sicile cette espèce est remplacée par deux autres formes, dont l'une à dernier tour plus bombé, à ombilic très-étroit, à bouche plus grande et circulaire, à stries fortes et peu régulières, comme celles de l'H. profuga, (H. subprofuga, mihi; de Avellino, etc.); l'autre encore plus globuleuse, à stries elégantes, contigües et régulières $(\boldsymbol{H}$. filograna, Villa $=\boldsymbol{H}$. Ara dasï, Mandralisca, de Sicile). 
Delix unifusciata, p'oiret - Coq. terr. et fut. de l'tisne, e! de Puris: Prodrome: Avril, 1801.

a) candidula, Studer - System. Verzeichn. 18ะ0, flag. 87; non Michaud. Minor, unicolor vel fusco-fascialu - Russmässter: Iconogr. d. Land. und Süssw. Moll. VI, 1837 ; pl. 25 , tig. 350.

B) alpicola, mihi - Minor, tenuiuscula; apertura magis rolundata; apice corneo-fusco, fasciis angustis, pallidis, interruptis, evanescentibus (II. alpina?, Megerle; non Faure-Big.) Hitut. 4,5; Diam. 6,5 mill.

y) gratiowa, Studer - Major; quoad colorem et cretcra ut in 11 . candiclule, Stud. - Haut. 6 a 7 ; Diam. 10 à 11 mill.

V. G. - Vallée de la Doire Ripaire: Suse, Chiomont, S. BerIrand, Oulx, Mont-Cénis à la Grande Croix, B00-1880 (Stabile, Strobel, Mella, Mortillet); Bardonnèche, $1230^{\mathrm{m}}$; au-dessus de Clavières, route du Nont-Genève, $1790^{\mathrm{m}}$ (Strobel) (Var. $\propto$ ); au-dessus de Rochemolles (Monte-Thabor) $\mathbf{1 8 0 0 - 1 9 0 0 \mathrm { m }}$, roches gneissiques (Stabile) (VAR $\beta)$.

R. P. - Plaine du Pò (nord): Turin, $250^{\mathrm{m}}$ (Mortillet); MezzanaCorti près la Cava, $80^{\mathrm{m}}$ (Strobel) - $\Lambda$ ssez probablement ici erratique! Plaine du Pó (sud): Valenza, Alexandrie et Val-Madonna, Marengo,

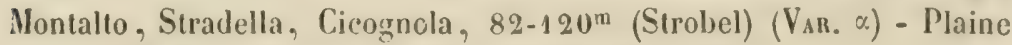
et au pied des collines de la Serivia (Mortillet) (Van. $a$ ).

V. T. - V. de la Stura de Cuneo: Rocea-Sparvera, Gajojla, Vignòlo, près Cuneo, \$00-700 ${ }^{\mathrm{m}}$ (Mortillet) (Var. $\alpha$ ) - Val-Vermegnana: Col de Tenda, $1870^{\mathrm{m}}$ (Mortillel) (Var. $\alpha$ ) - Val de la Scrivia: Tortone, Corniasca, Serravalle (route de Gènes), sur les monts et les cơteaux, les Var. a et $\beta$. (Mortillet) - Val Staffora; Val Coppa; Val Aversa; Val du Tidone; Val Trebbia: Bobbio, Var, $\alpha$, et $\beta$. (Strobel).

Obs. Vit sur les pelouses, sur les petits arbustes, monte au pied des rocher's, ete.; plus ordinairement parmi les lierbes. 


\section{Hellx conspurcata.}

Hetix conspurcula, Draparnaud - Tabl. Moll. 1801; el Hist. Moll. 1805, pl. VII flg. $23-25$.

R. P. - Collines transpadanes: Sciolze à l'est de Turin, terrain éocén. (Mella); Rosignano à sud-est de Casale, 200-300m (Mella); Casale-Monferrato (Cesati).

Sect. C A M Y I A

(Campylea, Beck - Ind. Moll. 1837 ; pag. 24.)

a) CIngulifera, Held - in: Isis, 1837; pag. 941.

\section{Helix glacialis.}

Melix glacialis, Thumas - in Férussae: Tabl. System. 1822; et Hist. Moll. 1818 11. 67 , fig. 2.

V. C. - Vallée de la Doire Riparia: Monte-Thabor, au-dessus du village de Rochemolles, région gneissique, $2100^{\mathrm{m}}$ (Stabile); MontCénis, à la Ramasse, $2000^{\mathrm{m}}$ (Mortillet, Cesati, Bellardi) - Val Stura de Lanzo: régions élevées $\left(1600-1800^{\mathrm{m}}\right)$ de la Val d'Ala, au-dessus de Balme etc.; sous les pierres crystallines, associée à la Vitrina major, à la Hyal. fulva et Petronelle, etc.; près les ruisseaux, ou près les neiges lorsqu'elles ne sont pas encore complétement disparues (juillet, août, septembre). Epiphragme vitreux (Stabile).

M. ${ }^{\mathrm{r}}$ de Mortillet cite cette espèce aussi du Monte-Rosa; mais ni moi, ni M. ${ }^{r}$ Villa, nous ne l'avons pas trouvée!

Obs. Nâchoire large de 1 millimètre, très arquée, non dilatée aux deux bouts qui sont légèrement anguleux, médiocrement robuste,

(a) Màchoire à còtes ( 4 a 6 ) saillantes, paralleles, écartées, et à bord denté. Vesí cules muqueuses 2 , simples ou bifides. Dard 1 , arqué, biangulcux à sa base. 
l'un faure corné vers le bord libre, d'un jaune d'ambre sale verg le bord opposé; clle presente trois côtes médianes fortes, saillantes, (et une quatrième peu prononcée) un peu rapprochées, presque droites, verticales, qui correspondent a un égal nombre de denticulations ou crénelures assez prononcées, obtuses. On remarque aussi sur cet organe, des rides transversales, fines, serrées, parallèles, sinueuses, apparentes. - La langue de l'H. glacialis est longue de 4 millim.; large de 1; légèrement dilatée vers le bord antérieur qui est trèsarrondi; elle présente des rangées très nombreuses, parallèles, de dents; celles-ci sont plus grosses anterieurement, un peu obtuses au bout. Cet organe est plus développé que celui de l'H. colubrina, Jan (Communiqué par M. ${ }^{r}$ de Saint-Simon).

(5) Corneola, Held - in: Isis; 1837 , pag. 911.

\section{* 26. Helix monata.}

Helix zonuta, Stader - System. Verzeichn. etc. in Schweiz. Conch.; 1820, pag. 87; nun: Ilel. zonala, C. Pfeiffer.

- zonala (partim), Vérussuc - Hist. Moll., 1819-21; pl. 68, fig. 8.

- potens (partim), Deshayes - in Férussac: Hist. pag. 25; 1839.

- viltala (olim), Villa - in schedis, et fide ipsius; non H. vittata, Cristoforis et Jian. (a)

- zonalt, L. Pfeiller - Monogr. Helic. vivent. 1; 1848, pag. 359.

\section{a) typiea -}

V. C. - V. de la Toce: Simplon, 1000-1300m (Stabile) -- V. Anzasca: Calasca, $800.600^{\mathrm{m}}$ (Stabile) - V. Cannobina, montagnes de Cannobio, au lac Majeur, 400-600 ${ }^{\mathrm{m}}$ (Villa) - V. d'Aosta, 600-1200 (Mortillet) - V. de la Stura de Lanzo: au-dessus de Ala, roches rrystallines, $1100^{\mathrm{m}}$ (Stabile) - V. de la Doire Riparia: (Val-Bardonnèche) Plan du Col , $1300^{\mathrm{m}}$ (Mortillet); Pas des Echélles, $1790^{\mathrm{m}}$ (Strobel). 
9) Tavovirems, Dumont et Murtillet - Hist. Holl. de Savoie, 1S3z, prospoctus, pag. 3 ; et: Cat. crit. et malacostat. Moll. Savoie, etc. 1857; p. 77 Testa solidiuscula, vix pellucida, flavido-viridescenti, absque fascia.

Parmi les blocs accumulés à côté de la cascade de la Cénise (sur le Mont-Cénis, $1800^{\mathrm{m}}$ ) (Mortillet).

Obs. Les dimensions de celte espèce varient notablement, et souvent dans le même endroit, mais la forme est toujours constante. Dans l'haute vallée du Tessin au-dessus du Dazio Grande, route du S. Gothard, j'ai trouvé des individus qui avaient une hanteur de 14, et un diam. de 25 millim.; et d'autres dont l'haut. était à peine de 10 , et le diam. de 17 millimètres.

\section{2ซ. Melix foetens.}

IIelix fotens, Studer - System. Verzeichn. etc., 1820 ; pag. 87.

- fotens (altera pars), Deshayes - in Férussac: Hist. Moll.; pag. 25 , pl. 69 , A. fig. 4. - non Helix foetens, Russmïssler: Iconogr. d. Land und. Süssw. Moll. Il, 1835; fig. 92; nec Rossm. (loco citato), X; 1839, pag. 12, fig. 600 .

VAr. clsalpina, mihi $-($ Helix adelozona, Parreyss; et rhatica, Mousson teste Strobel.)

Testa late umbilicata, orbiculato-depressa, corneo-flavescens, pellucida, nitida, glabra, vel sub lente perminutissime granulata, oblique crebro-striatula, supra medium fusco-unifasciata, fascia latiuscula, infra zonula pallido-flavicanti, pellucida cincta; interdum fascia altera inferiore fusca, valde diluta, margini vero peristomatis macula

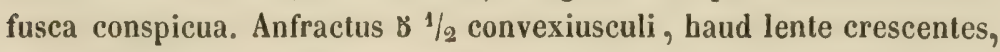
ultimo antice deflexo. Apertura perobliqua, transverse rotundato-ovalis; peristoma reflexum, intus labiatulum, marginibus approximatis, convergentibus; supero expanso, sub-simplici, columellari latiusculo dilatato, paululum supra umbilicum reflexo. $(=H$. adelozona, Parreyss). Specimina pedemontana et longobardica, 
NB. Interdum ociurrit lesta crassiori, badia, depressiuscula, fascia? pallida, nulla vel imonspiena (II. rhetica, Mousson, leste Strobel). Specim. longobard. lantum.

Coquille largement ombiliquece, déprimee, peu convexe en dessus, assez conrexe en dessous, de couleur cornée un peu jaunatre ou olivàtre, mince, quoique sulide, assez luisante, demi-transparente, glabre el, à la loupe, très-finement granulée, à stries obliques, fines, marquées, avec une bande brune-rougeàtre au liers supérieur du dernier tour, accompagnèe en dessous d'une zone blanc-jaunàtre, pàle, peu apparente, inféricurement à laquelle on aperçoit souvent une autre bande large, rougeâtre lavée et à peine visible; la marge externe supérieure du péristome est bordée d'une tache brune-rougeàtre dilatée en dessus et en dessous de la bande principale et simulant un commencement de deux zones supplémentaires. Spire de $\$ 1 / 2$ tours, croissants un peu rapidement, à sulure médiocre et sommet obtus; dernier tour descendant en dessous. Ouverture très-oblique, transversalement ovale. Péristome interrompu, réléchi, à bourrelet interne trés peu épais; bords rapprochés et convergents; bord supérieur simple, très peu rebordé; columellaire un peu evasé, rébordé et un peu largement réfléchi à l'extrémité sur l'ombilic. Epiphragme à l'entrée de la coquille, mince, transparent, lisse, un peu miroitant, membraneux, avee une tache blanchâtre, opaque, crétacée, répondant à l'orifice de la respiration. Il existe quelquefois un autre épiphragme enfoncé dans la coquille, qui ressemble à une pellicule blanche el opaque.

$$
\begin{aligned}
& \text { Hauteur: } 11 \text { millin. | specimina Pedemontans. } \\
& \text { viametre: } 26 \text { millim. }
\end{aligned}
$$

ß) Virescenti, pellucida, unicolore, fasciis nullis. (Anomaliat

V. C. - V. Doire Baltea: Liliannes en Val de Lys, ou Gressoney, $600^{\mathrm{m}}$; (micaschistes et gneis).

Obs. J'ai nommé cisalpina la variété de l'II. fotens de Studer, toujours plus grande, plus ou moins solide, qui habile les vallées uéridionales alpines et préalpines, et qui parait bornée à la chaine centrale alpine, entre le $4 \mathrm{~B}^{\circ}, 50$ et le $46^{\circ}, 10$ de latitude; et le $2 \mathrm{~B}^{\circ}$ 
et $28^{\circ}$ de longitude: Val Gressoney ou Val-de-Lys, roches gneissiques, $600-800^{\mathrm{m}}$ (Stabile); Val-Sassina (Stoppani); Val Brembana (Strobel) Haut. 11, Diam. 26 mill. Tantôt elle devient plus épaisse, plus grande, un peu plus déprimée, de couleur rougeâtre, sub-opaque, et la zone pàle en dessous de la bande brune est presque effacée (H. rhotica, Mousson, teste Strobel) - Haut. 12, Diam. 29 millim.: Valtelline supérieure (Mousson); Val-Brembana (Villa); Val Camonica (Strobel). L'élévation sur le niveau de la mer, de toutes les localités lombardes citées, varie entre $300-600^{\mathrm{m}}$; la nature du sol est calcaire-dolomitique, la Valtelline exceptée, qui est presque entièrement gneissique et micaschisteuse.

L'H. foetens, Studer, (type, ou transalpina) n'a d'autres différences que une taille constantement plus petite (Haut. 10, Diam. 22 mill.) le test est plus mince, la spire un quelque peu plus élevée. Elle est très peu répandue, quoique assez abhondante, et paraît limitée à une quelque vallée du versant septentrional des Alpes en Suisse. M. ${ }^{r}$ François Venelz (fils) en sa qualité de naturaliste infatigable, d'ingénieur des chemins de fer, et des ponts et chaussées, n'a rapporté de ses nombreuses excursions dans les montagnes suisses, que l'H. zonata, Studer; et du Mont-Catogne (côtẻ sud qui descend dans la Val de Ferret (Suisse); micaschistes et gneis); et des environs de Saint-Pierre $\left(1500^{\mathrm{m}}\right.$; micaschistes, gneis et schistes gris) dans la vallée d'Entremont (a), l' $H$. foetens de Studer.

Or c'est justement les exemplaires de la vallée d'Entremont que feu M. ${ }^{r}$ I. Venetz (père) envoyait à feu le prof. Studer, et que ce professeur nommait $\boldsymbol{H}$. fotens $(b)$. Voici ce que dit en propos $M{ }^{r}{ }^{r}$ Studer mème "Un peu plus pelite que l'H. zonata, mais toujours une fois

(a) La Val de Ferret n'est que un embranchement, (qui s'ouvre près le village d'Orsières) de la vallée de la Dranse, ou d'Entremont, versant nord ou suisse du Gr. S. Bernard.

(b) Avant de commencer ce travail, ne connaissant pas la vraie $\boldsymbol{H}$. foetens, Studer, j'envoyais à quelques-uns de mes amis et correspondants cette même coquille de la vallée d'Entremont sous le nom de $\boldsymbol{H}$. adelozona, Parreyss; VAR: Venetzii, mihi; et plus tard (sur l'autorité de quelque savant conchyliologiste) sous le nom d' $H$. planospira, Lk., Var. Venetzii. Je prie donc ces messieurs de corriger leurs ètiqueltes en: H. folens, sluder. 
plus grosse que l'I1. cornea, Drap. el plus brillante, couleur brunsâle. Outre la bande peu marquée, on voit le commencement d'une seconde. L'animal, dit-on, répand une odeur désagréable quand on le sort de sa coquille $(a)$. Saint-Branchier $(b)$ en Valais (Venetz)".

De tout ce que nous venons de dire il s'ensuit que l' $\boldsymbol{H}$. foetens de Studer et l' $I$. zonata de Studer, sont deux choses différentes, et qu'on ne doit donc pas les ranger en synonyme l'une de l'autre, comme l'on fait plusieurs conchyliologistes, quand même on ne voudrait les considérer que comme de simples variétés locales de l' $\boldsymbol{H}$. zonata. En vérité que si l'on compare ces deux formes, abstraction faite de toute considération malacostatique, on peut bien les retenir comme deux variétés, l'une plus globuleuse, l'autre plus mince et déprimée, etc.; mais quand on voit cette dernière ( $\boldsymbol{H}$. fœetens) dans nos vallées cisalpines acquérir une taille plus grande, plus robuste et son domaine être beaucoup plus étendu, que devra-t-on conclure?... Que l' $\boldsymbol{H}$. foetens est une espèce distincte et, plutôt que du versant nord alpin, elle est indigène de la zone méridionale inférieure, laquelle espèce ayant franchi les Alpes, est descendue dans telle ou telle autre des vallées transalpines, où les conditions physiques et naturelles ont été plus ou moins favorables à son parfait développement (c).

Quelques auteurs ont, un peu mal à propos, associé l'H. foetens de Studer à l'H. ichthyomma, Held, du Tyrol $(d)$, de la Carniole, etc.; cette espèce est, à vrai dire, bien voisine de l' $H$. foctens, mais elle est constantement petite, moins convexe en dessous que l' $H$. foetens, de couleur brun-rougeàtre, assez épaisse, peu transparente; la zone blanchâtre est plutôt lailigincuse que hyaline, et plus prononcée; l'autre bande brune inférieure plus visible, de la mêne couleur que le reste de la coquille, le bord collumellaire du péristome moins renversé sur l'ombilic. L'H. achates Ziegler = cingulina, Deshayes (in Férussac - Hist. Moll.) du Tyrol allemand, de Schneeberg en

(a) Cette odeur flesagriable n'est pas exclusive de l'H. fotens; même l'H. zonala, la IIyalina cellaria, etc. répandent cette odeur ripoussante.

(b) Saint-Branchier est un village de la vallée d'Entremont, à $760 \mathrm{~m}$.

(c) $\mathrm{M}^{\mathrm{T}}$ le prof. Mousson a trové l'Hel. adelozona dans l'Engadine.

(d) D'apris M.r Blanchet de Lausanne, celte espece aurait été Irouvie par M. ${ }^{r}$ Mousson à Tarasp (basse Encribline), c'est à dire peu loin des frontieres du Tyrol. 
Autriche, et même associée à l' $H$. ichthyomma, en Carniole, n'est qu'une varieté de cette dernière espèce, très aplatie des deux côtés, et dont la zone blanche est très distincte, la bande brune plus dilatée et confondue. L' $H$. fotens, Stud., l'H. adelozona, Parr., l' $H$. rhoetica, Mousson, sont trois formes variantes en dimensions, en solidité, même en couleur, mais c'est toujours la même chose que l'on a sous les yeux; tandis que si l'H. ichthyomma, Held a quelques ressemblances avec la foetens, la Var. achates s'en éloigne tellement qu'il est impossible, même aux conchyliologistes les plus scrupuleux de la réunir à la foetens en une seule espèce! Les belles figures données par Rossmässler (Iconogr. Heft II, fig. 92; et Heft X, fig. 600) ne représentent pas I' $\boldsymbol{H}$. foetens (type) du Valais; mais l' $H$. ichthyomma, Held, et sa variété achates.

\section{Helix umbilicaris.}

Helix hispana 9, Linné $(a)-$ Syst. Nat. Ed. X, 1758; I, p. 772, N. 599; (teste Beck).

- planospira, Lamarck - Hist. Anim. sans vertébres; Tome VI, 2.e partie, Avril 1822. (exclus. H. cryptozona, Ziegler) $(b)-$ Non $H$. planospira, Michaud Compl. à Draparnaud, 1831, pl. XIV, fig. 3-4); nec Philipıi (Enum. Moll. Sicil. II, 1844); nec L. Pfeiffer (Mon. Hel. viw.; I, 1848; Addenda, p. 449.)

- villata Cristoforis et Jan - Cat. rer. nat. in Mus. extant, 1832 (teste Rossmässler): non vittata, Nüller; nec Villa.

- umbilicaris, Brumati - Catal. sistem. Conch. di Monfalcone, 1838; non Olivi (Zool. adriat. 1792, pag. 177) (c).

VAR. Padama, mihi -

Testa aperte umbilicata, orbiculato-convexa, spira parum elata, apice obtuso, cornea, vel corneo-olivacea, subpellucida, nitidiuscula,

(a) Dans quelques ouvrages, et dans plusieures collections cetle espèce porte, comme nom plus ancien, le nom d'H. hispana, Linné; il paraît qu'il ait été adopté d'après l'autorité de Beck seulement; d'ailleurs la phrase linnéenne (a Testa umbilicata, convexa; anfractibus 5 teretibus, umbilico tenui. - Habit. in Europa australi. Testa colore corneo, ) désigne une campylée coruée quelconque. Nous avons déjả une $I$. hispanica, Partsch, et une $H$. hispanica, Michaud; une $H$. hispana, Linné, et tant plus pour une espèce qui est tout autre que espagnole, ne pourrait donc pas être adoptée que à confusion de la nomenclature déjà si embrouillée!

(b) L'H. cryptozona, Z. n'est qu' zne simple varieté - minor, tenuis, diaphana . de I' $H$. macrostoma, Mühlfeldt (Habit. la Sicile).

(c) L'H. umbilicaris, Olivi, est une varieti de l'H. ericetorum, Müller. 
ublique striatula et, sub lente, striis spiralibus confertis minute decussata; supra medium, in pallido-albida vitta, fusco-unifasciata; superius fascia altera fusea, diluta, cito evanescente. Anfractus $\$ 1 / 2$ convexiusculi, sutura mediocri distincti; ultimus antice breviter deflexus. Apertura rotundato-lunaris, obliqua; peristoma albido-labiatum, mediocriter reflexum, marginibus remotis, columellari crassiusculo, circa regienem umbilicarem late dilatato, atque ad tertiam ultra partem umbilicun subtegente.

Coquille assez largement ombiliquée, globuleuse-déprimée, médiocrement convexe en dessus, bombée en dessous, couleur de corne plus ou moins pàle ou olivâtre, assez solide, un peu luisante, demitransparente, à stries obliques d'accroissement peu régulières, et à surface cliargée de pointuations oblongues, ou petites lignes saillantes, disposées transversalement, addossées et paraissant quelque peu ondulées, visibles même à un faible agrandissement; à la partie supérieure du dernier tour une zone brun-rougeâtre au milieu d'une bande blanchâtre ou corné-pâle, et en dessus de celle-ci une autre zone plus étroite, plus prononcée vers le bord du péristome, et s'évanouissant ensuite insensiblement. Tours de spire $b^{1 / 2}$ médiocrement convexes, à suture assez distincte et à sommet obtus; dernier tour descendant un peu en dessous. (a) Ouverture oblique, ovale-arrondie, plus large que haute; péristome réfléchi, à bords écartés, et à bourrelet interne blanc; bord inférieur, ou columellaire, plus épais; à l'extrémité, vers la région ombilicale, dilaté en forme de languette et recouvrant presque la moitié de l'ombilic.

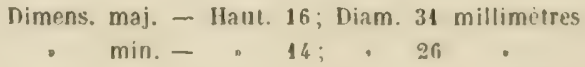

V. C. - Val-Pellice: la Torre de Luserna $(b), \mathbf{B} \$ 0^{\mathrm{m}}$;

R. P. - Haute vallée du Pô: Calcinéro au-dessus de Paesana,

(a) Les anneaux, ou espéce de cèrcles d'un blanc jaunâtre (désignant le nombre tes fois que le mollusque a interrompu te travail de construction de sa cuquille) si bien prononcés, à de distances irrégulières, sur l'IJ. umbilicaris tynique, sont presque nuls darıs notre varieté Padana.

(b) C'est la derniere des vallées cispadanes; on masse l'iei dans la vallée du pó. 
700-800 ; terrain crystallin (Stabile); Brondello au-dessus de Saluzzo (Mella); entre la Val Pellice et la vallée du Pô (Villa).

V. T. - Val-Varaita: Venasca, $850^{\mathrm{m}}$; (Mortillet).

Obs. Qu'est-ce que l'H. planospira, Lamarck? Voilà une question agitée depuis long temps parmi les naturalistes! Qu'il soit permis d'abord de jeter un coup d'œil sur les divers ouvrages dans lesquels on parle, dans un sens ou dans l'autre, de l' $H$. planospira; nous verrons ensuite à une conclusion! M. Michaud (Complément à Draparnaud; 1831) sous le nom collectif d'H. planospira Lamarck, a-t-il voulu comprendre deux espèces différentes: l' $H$. zonata, Studer et l'H. umbilicaris, Brumati? La phrase latine, la description, les figures données par M.' Michaud, et l'indication "Alpes " indiqueraient un peu l'H. zonata, Studer; c'est la convinction aussi de MM. ${ }^{\text {rs }}$ l'abbé Dupuy (a), Moquin-Tandon (b), Drouët (c), Mortillet $(d)$; mais le derniers mols de sa diagnuse "plus grande, plus aplatie, plus lisse, etc. " et la cilation des autres localités: haute Autriche, Frioul, etc. se rapportent sans doute à l'H. umbilicaris, Brumati. Ainsi la fig. 0, tab. 3 de Gualtieri représente cette dernière espèce, tandis que l'ouvrage de Studer cité par M. ${ }^{\mathrm{r}}$ Michaud, ne peut avoir rapport que à l'espèce de la Suisse, c'est à dire à l' $H$. zonata. II parait cependant que M. ${ }^{\mathrm{r}}$ Michaud sous le nom de $H$. planospira, Lk. ait voulu décrire l' $H$. umbilicaris.

M. ${ }^{\mathrm{r}}$ Rossmässler (Iconogr. der Land-und Süsw. Moll. Heft. II, 1835; fig. 90) sous le nom d'H. planospira, Lk, donne une bonne description de l'H. umbilicaris, Brumati, de la vallée de l'Isarco, de l'lllyrie, des environs de Trieste, des Alpes Autrichiennes, de l'ltalie $(e)$; les figures, les diagnoses et les localités conviennent exactement à l' $H$. umbilicaris, même pour les localités italiennes (car

(a) Hist. Natur. des Moll. terr. et d'sau douce qui vivent en France; II fasc. Janvier, 1848.

(b) Hist. Natur. des Moll. terr, et Muv. de France; 1855.

(c) Enumération des Moll. terr. et fluv. viv. de la France continentale; 1855.

(d) Catal. crit. et malacostat. des Moll. terr. et d'ear douce de la Savoie, etc.; 1857.

(e) M.r Rossmässler ajoute aussi : " la Sicile " mais plus tard (1838), il fait justement observer que l'helice sicilienne n'est pas l'H. planospira, I.k., mais l'H. macrostoma, Mühlfeldt (= siculina Ziegler). 
celte espèce, sauf quelques legères modifications, on la rencontre dans le Véronais, le Vicentin, le Bellunais, le Padouan, à Masse-Carrare, à Livourne en Toscane, et dans l'Etat Romain). Le même auteur (Heft. VIII, 1838, fig. B03) cite encore une forme un peu variée d' $H$. planospira, Lk. des environs de Trieste; la spire est un peu plus élevée, l'ouverture un peu plus arrondie. En Carniole, el dans les environs de Trieste, l' $H$. umbilicaris présente très souvent une coquille plus petite, à spire un peu élevée, quelquefois mème subglobuleuse (Trieste); les bandes sont moins prononcées, ou bien nulles ou presque nulles, la couleur du test moins gaie et brillante, et plus terne $(a)$; mais c'est toujours le mème type! Au contraire dans l'haute vallée du Pô, nous avons notre variété Padana, grande, plus globuleuse en dessus et, proportionnément plus bombée en dessous, à test assez solide et granulé, à bord columellaire du péristome renversé sur un bon tiers de l'ombilic, etc. De l'autre côté du Pó, sur les “Colli Euganei " dans le Padouan, notre $H$. Padana paraît déjà; ses pointuations allongées, ou rugosités sub-ondulées et serrées, sont bien prononcées, mais elle est petite, mince, d'ailleurs la couleur du test d'un bel corné-fauve, c'est la couleur de l'umbilicaris type des régions environnantes. M. ${ }^{r}$ Deshayes l'illustrateur de Férussac, (in Férussac: Hist. Moll.; 1839-40), après avoir (pag. 27), à bon droit, retirée de l' $H$. zonuta, Férussac, plusieures espèces diverses que II. Férussac y avait comprises, a réservé le nom de zonata, Férussac (non Studer) à une espéce du Frioul, de l'lllyrie, de Monfalcone près Trieste, de Pise et Florence. Les figures de Férussac (pl. 68, fig. 10, b. c. soit dextre et sénestre, non celle du milieu) et l'habilat cités par M.' Deshayes conviennent à l'H. umbilicaris, Brum., laquelle, comme nous l'avons dit ci-dessus, descende jusque dans l'ltalie méridionale; cependant les descriptions données par M. ${ }^{r}$ Deshayes se rapportent plutòt à une forme moins déprinéce que celle du Véronais, du Vicentin et méme de la Toscane, et à bandes moins prononcées; nous avons déjà fait mention de notre

(a) J'ai distingué dans ma collection cette forme d' $\boldsymbol{H}$. umbilicaris par lo nom de Illyrica; et culle a spire plus déprimée, à test d'un corné plus vif et à bantes liéll marquires, par le nom de llalica. 
mutation illyrica, ef ce sera, peut-être, sur des exemplaires de cette variété que M. ${ }^{\mathrm{r}}$ Deshayes aura fait sa description; les quelques individus d' $H$. umbilicaris que j'ai reçus de Masse-Carrare, et de Lucques sont aplatis en dessus, comme ceux du Véronais, de Recoàro dans le Vicentin, du Bellunais, etc. Mais, à page 23, M. ${ }^{\mathrm{r}}$ Deshayes, sous le nom d'H. planospira, Lk. (a) donne la description d'une autre espèce de l'Italie méridionale, de Sicile, de Morée, etc. On voit évidemment, d'après l'habitat, la citation des auteurs, etc. qu'il a reuni ensemble deux espèces: $l^{\prime} H$. umbilicaris Brum., et l'H. cryptozona, Ziegler. En fait ce n'est que plus tard que les auteurs ont séparé et érigé en espèce distincte l' $H$. macrostoma, Mühlfeldt (dont l'H. cryplozona n'est qu'une simple variété). L'un des caractères différentiels que $M{ }^{r}$ Deshayes donne à son $H$. planospira, est la granulation régulière de l'épiderme; cette observation est juste; l'H. umbilicaris du Véronais, du Vicentin, du Bellunais, du Frioul et de l'lllyrie est toujours lisse, ou avec des granulations très peu distinctes, méme à un convenable grossissement; au contraire l' $H$. umbilicaris de Masse est déjà plus granulée, celle de Lucques, outre les granulations, présente sur les premiers tours de spire quelques rares poils; et cependant c'est toujours la même espèce, la même forme, c'est la vraie $H$. umbilicaris, quoique quelqu'un des nos conchyliologistes l'ait désignée pour $H$. setipila, qui est bien différente! Notre variété Padana, de l'H. umbilicaris, tant celle de l'haute vallée du Pd, ou Pedemontana, que celle des "Colli Euganei " ou Euganea, est très bien granulée en forme de stries courtes et transverses. Il parait que la granulation de l'épiderme et les poils soient l'un des caractères méridionaux des Campylées à test corné: l'H. setipila, Ziegler de l'Abruzze el du Napolitain; l'H. setosa, Ziegler, de la Dalmatie; l'H. subzonata, Mousson, de Cephalonie; l'H. comephora, Bourguignat, de Morée; l'H. cyclolabris, Deshayes, de Grèce; l'H. pellita, Férussac, de Rhodes, etc. sont toutes des espèces à coquille granulée, et plus ou moins garnie de poils.

(a) M.r Deshayes cite encore pour cette espece la même figure de l' $\boldsymbol{H}$. zonata, Fér. (pl. 68 , fig. 10 , b. c.) 
I'n mot, à présent, sur l'H. planospira, Lk. de la troisième édilion de Lamarck, par M. ${ }^{\top}$ Deshayes: il est évident que, s'agissant d'un ouviage général, ce professeur a compris, sous le nom collectif de planospira, plusieures espèces qui ont quelques affinités entre elles (H. zonata, H. umbilicaris, $\boldsymbol{H}$. macrustoma, etc.).

L. Pfeiff. (Monogr. Helic. vivent.; Vol. I, 1848) après avoir, à p. 347, sous le nom de planospira, Lk. donné une bonne diagnose de l'H. umbilicaris de l'lllyrie et de l'Italie boréale; à page 449, dans l' $A d$ denda, change le nom de planospira, Lk., en celui de hispana, Linné; la synonymie cependant est la même (H. umbilicaris, Brum., H. planospira, Rossm., etc.) Quant à l'H. planospira, Lk.; il en fait une espèce séparée, intermédiare, comm'il dit, entre l' $H$, hispana, Linn. et l' $H$. zonata, Stud., et ayant beaucoup d'analogie avec l'H. macrostoma, Mühlf.; et lui donne en synonyme le noin d' $\boldsymbol{H}$. planospira, Michaud! Serait-ce notre variété Padana?; cependant les dimensions de notre Padana sont majeures que celles données par M. ${ }^{\mathrm{r}}$ Pfeiffer à son $I I$. planospira. Quoi qu'il en soit, le nom d' $H$. planospira, Lk. ne pourrait pas etre adopté pour notre $H$. Padana.

J'ai laissé le dernier, et mon sans raison, celui que je devais placer avant tous les autres, l'auteur même d' $H$. planospira, l'illustre Lamarck. Que dit-il cet auteur $(a)$ ? La phrase latine et la citation de la planche $\mathbf{3}$, fig. 0 , de Gualtieri laisseraient nullement douter qu'il s'agisse de l'H. umbilicaris, Brum.; mais si, d'après ce qu'en dit M.' Deshayes (in Férussac, pag. 23), l'H. planospira, Lk. porte aussi le nom d'H. cryptozona, Ziegler; nous aurions mème ici deux espèces sous le même nom; cependant, dès qu'on a érigé en espèce distincte, et assez connue, l'H. macrostoma (dont $1 H$. cryptozona n'est qu'une variété à test moins solide), le nom de planospira, Lk. resterait donc tout seul à l'autre espèce italienne à spire deprimée,

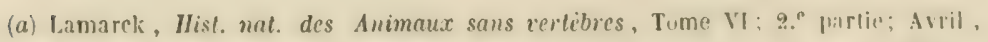
1822. N. 48; II. planospira a II. testa orbiculato-depressa, subtus convexa, umbilicata, glabra, corneo-lutescente; spira plana; ultumo anfractu fascia albida rufu marginata cincto; labro margine reflexu, albo. - Gualtieri: Test. t. 3, fig. O. - H. zonatı. Daule, hard de Firnssar.: Mist. Moll. N. 163 . HabIt. en llalip - Diam. enviruns 10 lignes (I.amarek). 
c'est à dire à l'H. umbilicaris, Brum., ou plus parliculièrement ả la mutation italica.

En résumant, nous dirons: $10^{\circ}$ Que le nom d'H. planospira, Lk. ne peut désigner absolument d'autres espèces que l' $H$. umbilicaris, Brumati, et l'H. cryptozona, Ziegler; 2. ${ }^{\circ}$ Que cette dernière espèce ayant été retirée et separée, on pourrait bien, suivant les lois d'an. tériorité, adopter le nom d'H. planospira, Lk. (comme plus ancien de umbilicaris, Brum.) pour l'autre espèce; cependant, à fin d'éviter toute confusion, il est préférable de le faire passer en syno. nyme de l'H. umbilicaris, ou plus proprement pour en désigner la mutation à spire aplatie, ou italica; $3 .^{\circ}$ Que l'H. umbilicaris, Brumati; l'H. zonuta, Fér. de M. ${ }^{r}$ Deshayes; l' H. planospira, Lamarck (exclus. H. cryptozona); l'H. planospira, Lamarck, de M. ${ }^{r}$ Rossmässler, ne sont qii'une seule et même espèce, à spire plus ou moins déprimée, à test plus ou moins corné-pảle ou rougeàtre, à épiderme plus ou moins lisse ou granulée, suivant les différentes localités; $40^{\circ}$ On pourrait donc rétablir de la manière suivante la synonymie des Campyleis italiennes appartenantes au group de l'H. zonata:

1. Helix zonata, Studer (non Fér., nec C. Pfeiffer).

Habit. Les Alpes de la Suisse, du Piémont et de l'haute Lombardie.

2. Iellx foetens, Studer ( $\boldsymbol{H}$. foetens (partim), Deshayes, in Férussac; non $\boldsymbol{H}$. foetens, Moquin-Tandon.

a) transalpina, minor, tenuis, (type de Studer).

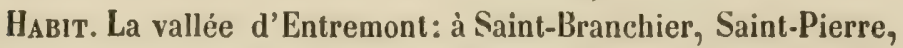
Mont-Catogne (Cant. du Valais en Suisse).

B) cisalpina, mihi - Major, solidiuscula (H. adelozona, Parreyss, in specimin), interdum crassa, badia (H. rhoetica, Mousson, teste Strobel).

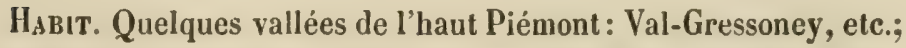
et les vallées lombardes: Val Brembana, Val Camonica, Valtelline d'où serait descendue un peı de l'autre côté: Engadine (Mousson). 
3. Mellx Iehthyomma, Held. (II. foetens (partim), lPeifier, Russmässler).

Habir. Tyrol, basse Engadine, Carniole, etc.

ß) achates, Ziegler ( $H$. cingulina, Deshayes, in Firussaci) Testa utrinque depressissima, zonula alba distincta, fuscia supera saturate-fusca, diluta.

HaBit. Carniole, Tyrol allemand, Schneeberg (Autriche).

4. Hellx umbilicarls, Brumati ( $H$. hispana?, Linné, teste Beck).

A. typica, (II. umbilicaris, Brumati; non Olivi = H. zonata, C. Pfeiffer; nun Studer $=\boldsymbol{H}$. zonata, Firussac (emend. Deshayes).

a) Illyrica, mihi - Testa plus minusve subglobuloso-depressa, glabra, perscepe pallide-cornea, fasciisque evanescentibus.

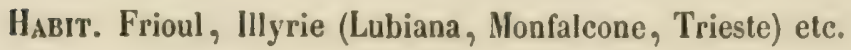

ß) Italica, mihi - (H. planospira (partim), Lamarck) Testa sepe majuscula, spira plana, corneo-rufescens, fasciis conspicuis.

* Testa glabra, umbilico latiori, patente.

$\mathrm{H}_{\mathrm{ABIT}}$. Véronais, Vicentin, Bellunais, etc.

* Testa minute granulata, margine columellari peristomatis paululum supra umbilicum latum reflexo.

Habit. Environs de Massa (Italie).

*** Testa minute granulata, et in primis anfractibus, pilis raris, erectis onnala.

$\mathrm{H}_{\mathrm{ABIT}}$. Livourne (Toscane).

B. Padana, mihi - Testa orbiculalo-convexa, spira panum clala, striis spiralibus confertim decussala, etc.; margine columellari peristomatis ad tertiam ultra partem umbilicum subtegente, etc. (Vide in descript.).

$H_{\text {Abit }}$. Ilaute vallée du Pó, au-dessus de Saluzzo; Val-Pellice.

B) Euganea, mihi - Differt (a Padana) testa minore, tenuiuscula, nitida.

HАвıт. "Colli Euganei " dans le Padouan (Martinati). 
\%. Hellx macrostoma, Mühlfeldı (H. siculina, Zieglet).

$\mathrm{H}_{\mathrm{ABIT}}$. Sicile.

ß) cryptozona, Ziegler - Minor, tenuis, diaphana.

$\mathrm{H}_{\mathrm{ABIT}}$. Sicile.

6. Helix setipila, Ziegler.

HABIT. Italie méridionale.

Sect. CHILOTREA A. (a)

(Chilotrema, Leach - Brit. Moll. pag. 106, ex Turton; 1831.)

\section{Helix Iapieida.}

Helix lapicida, Linné - System. Natur., Edit. X, 1758; I, pag. 768.

Carocolla lapicida, Lamarck - Anim. sans vertèbr., VI, 2. ${ }^{\mathrm{e}}$ part. 1822 ; Drap. - Hist. Moll. 1805, pl. VIl, fig. 35; Moq. Tand. - Hist. Moll. de France, 1855, pl. XI, fig. 22 à 24. (Anatom.).

V. C. - Vallée de la Doire Bàltea: (Val-de Lys, ou de Gressoney) Gressoney, $800-1200^{\mathrm{m}}$; terr. micaschisteux et gneissique (Stabile) - Vallée de la Doire Riparia: Suse, jusqu’à S.t Bertrand, B $00-800^{\mathrm{m}}$; terr. crystallin (Mortillet); Mont-Genève (Strobel).

(a) Mâchoire à côtes (6) un peu écartées, très saillantes et à bord fortement denté. Vésicules muqueuses 2, simples. Un seul dard, arqué, à pointe lancéolée. 
Sect. A I \ $\mathbf{A}(a)$

(Ariunta, Leach - Bibl. Moll., ex Turtur, 1831.)

\section{* 30. Helix arbustorum.}

Helix arbustorum, Linné - System. Natur., Edit. X, 1758; I, pag. 771.

Arianta arbustorum, Leach - loco citato; Draparnaud - Hist. Moll. , 1803; pl. V, fig. 18 - Ad. Schmidt: Geschlechtsapparat der Stylommatophoren; 1855 , pl. IX, fig. 70 (Anatom.).

V. C. - V. de la Doire Riparia: Oulx, route du Mont-Genève, parmi les buissons, lieux fraix; $800^{\mathrm{m}}$ (Stabile, Mortillet).

V. T. - Val-Maira, $1000^{\mathrm{m}}$ (Mella).

ß) alpestris, Ziegler - in Rossmässler: Icon. d. Land-und Süsw. Moll. 1837, Heft. V, fig. 297, C. $-H$. arbustorum, Var. alpicola, Férussac.

V. C. - V. Doire Riparia: Mont-Cénis, $2000^{\mathrm{m}}$ (Mortillet).

g) pleea, Ziegler - H. Wittmanni, Zawadzky; in Rossmässler - loc. cit., Heft. v, fig. 297 , d.

V. C. - V. de la Toce: Monte Rosa (Villa) - V. Doire Bàltea : Gressoney, $1663^{\mathrm{m}}$ (Mella) - V. Sésia (Val de l'Elvo): Oròpa, région micaschisteuse, $480^{\mathrm{m}}$ (Mella).

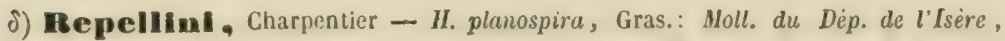
1840; non planospira Lk.; nec aliurum - Teslu majori, supra depressa, umbilico semiclauso (Haut. 14; Diam. 23 millim.) (b)

R. P. - Haute Vallée du Pò: à gauche du "Pian del Re " au Monte Viso, près les sources du Pô, $2000^{\mathrm{m}}$; roches crystallines et métamorphiques (Stabile).

(a) Màchoire à côtes (4-5) distantes, saillantes, et ả buril fortement denté. Vésicules muqueuses 2, simples. Un seul dard, arqué, bianguleux à la base, lanceolé à l'extremiti.

(b) J'ai vu dans la collection de $\mathbf{M}^{\mathbf{r}}$ de Gautard, a Vevey, un excmplaire de celte varieté determine comme $\boldsymbol{H}$. late-umbilicala, Jupuy. Ce num n'existe pas dans les auvrages de M. l'ablé bupuy et de Moquin-Tandon, sur les Mollusinues de France? 
Sect. T A C I E A. ( $(\varepsilon)$

(Tachea, Leach - Brit. Moll. 1s31; cx Turtur:;

* 31. Melix nemoralis.

Elelix nemoralis, Linne - System. Natur. Édit. X, 1758; 1, pag. 773.

6) Transalpina, milhi - Testa majuscula, fauce nigricanti-fusca, peristomate saturate-fusco. C'est la forme de la Suisse, de l'Allemagne et l'ute grande parlie de ta France.

V. C. - V. de la Doire Bàltea: Aosta, $\$ 90^{\mathrm{m}}-\mathrm{V}$. de la Doire Riparia: Suse, $600^{\mathrm{m}}$ (Mortillet). Rare!

B) cisalpina, mihi - Testa plerumque minore, fauce dilute infuscatı, peristomate rufescenti-fusco. C'est la forme commune des nos pays du revers méridional et oriental des Alpes (Piémont, Lumbardie, etc.).

V. C. - V. de la Toce: partie infẻrieure du Simplon, $310-700^{\mathrm{m}}$ (Strobel); Calasca en Val Anzasca, в00 ${ }^{\mathrm{m}}$ (Stabile); Orta, $370^{\mathrm{m}}$ (Marani); Val de la Doire Bàltea: Ivréa (Stabile), alentours du Lac Majeur, Arona, 200-600 ${ }^{\mathrm{m}}$ (Marani, Prada) - Vallée de la Doire Riparia: Oulx, Suse, $800-1000^{\mathrm{m}}$ (Stabile). - Val Pellice: Ia Tour de Luserne près Pinerol, $530^{\mathrm{m}}$ (Mortillet).

R. P. - Plaine du Pô (nord): Novara, Vercelli, Lomelline, Turin, 90-260m (Strobel, Mortillet) - Plaine sud: Stradella, Voghera, Asti, Dronéro $130-600^{\mathrm{m}}$ (Strobel, Mella).

V. T. - Val Stura de Cuneo: Val Vermegnana, route du Col de Tenda, de Borgo S. Dalmazzo jusqu’à Limone 600, $1000^{\mathrm{m}}$ (Mort.).

(a) Mâchoire á còtes (5 à 7); quatre très-fortes, et une plus faible au milieu; celles-ci constantes, et souvent six, et une au milieu ( $\boldsymbol{H}$. nemoralis), écartées, trés saillantes, et à bord fortement denté. Vésicules muqueuses 2, diviscées en 3 ou 4 branches simples. Un seul dard, à quatre arêtes tranchantes sur loute la longueur, en. furme de lance (H. Remoralis), ou un peu arq̨ue (H. sylvatica). 
f) apennina , mihi - H. Genuensis, purro; H. el'usca, cullectionum (a). Ceté forme se distingue par sa taille tres grande qui arrive quelquefors jusqu'a 30 millimetres de diametre, 't a 20 mill. de haut.

V. T. - Versant nord de l'Apennin - Val de la Bormida: Acqui (Villa) - Val de la Scrivia: Tortone, Ronco, Busalla (route de Génes) (Mortillet) - Val de la Staffora; V. Tidóne; Val Coppa : Casteggio; V. du Scurpasso; Val Aversa; Monte Pénice; Bobbio en Val Trebbia (Strobel).

Obs. La mutation à péristome blanc (que quelques conchyliologistes ronfondent ì tort avec l' $\boldsymbol{H}$. hortensis, Müll.) est assez rare dans les localites citées. M. ${ }^{r}$ Nella l'a trouvée à Dronéro; M. ${ }^{r}$ Hortillet dans la vallée de la Scrivia. Quant à l'H. hortensis, Müller, elle n'existe pas du côté sud des Alpes!

\section{Melix sylvatica.}

Helix sylvatica, Draparnaud - Tableau des Hollusq. 1801; Ul Hist. Moll. 1805, fl. VI, fig. 1-2.

V. C. - V. de la Doire Bälea: l'Allće-blanche au Col de Seigne, $2500^{\mathrm{m}}$ (Payol).

V. T. - Val Stura de Cunco: Val Vermegnana; Borgo S. Dalmazzo jusqu’ limone, roule du Col de Tenda (Mortillet).

(a) Ziegler, dans sa correspondance avec feu Charles Porro, savant naturaliste italien, déclara qu'il n'avait jamais institué ni l'H. etrusea, ni le Plunorbis etruseus! (Strobel: 


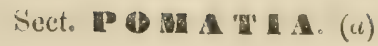

(Pomalia, Beck - Ind. Moll.; 1837 ; pa. 43.)

c) Cryptomphalus $(b)$, (partim), Igassiz - in: Charpentier - Moll. de le Suisse, 1837.

\section{Helix asmersa.}

Hetix aspersa, Muller - Verm. HisŁ. 1I, 1774, pag. 59; Férussac: Hist. Moll. Wl. 18.

V. C. - Val de la Doire Bàltea: Ivréa, 254m (Rezia) - V. Doire Riparia: Suse, jusqu'à S. Bertrand, 300-700" (Stabile, Strobel, Mortillet).

V. T. - Plusieures localités du Monferrato (Mella).

B) Ponatia, Leach - Brit. Holl., pag. 89; ex Turton, 1831 (c).

\section{* 34. Melix pomention.}

Helix pomalia, Linni - System. Natur. Édit. X, 1758, I, pag. 771.

V. C. - Vallée de la Toce: Pied du Simplon, Val Vedro, Val d'Ossola, 300-700 (Strobel); Lac Majeur, 200-500 (Stabile, Prada); Orta, $570^{\mathrm{m}}$ (Marani) - Val de la Doire Bàltea: collines de Viverone, (Vella) - Val de la Doire Riparia: Suse, Oulx, Bardonnèche jusqu'au hameau de la Roue, ̋̋00-1600 (Mortillet) - Val Pèllice: Tour de Luserne près Pinerolo, $830^{\text {m }}$ (Mortillet).

R. P. - Plaine du Pô (nord): Vercelli, les rives du Tessin (Strobel) - (sud): l'Astésan; plaine de Dronero et Val Maira (Mella); tout le versant padan de l'Apennin (Strobel).

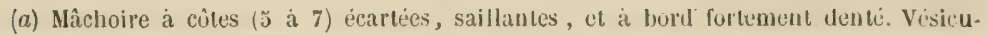
les muqueuses 2 , divisées en branches plus ou moins nombreuses, suivant l’âge du mollusque. Un seul dard courbe, avec quatre arêtes tranchantes, sur tunte la longueur.

(b) Chaque vésicule muqueuse divisie en 8 a 20 branches.

(c) Chaque vésicule muqueuse divisce en 20 a 40 branches. Les vísicules muqueuses de l'Hel. lucorum sont de la moitió plus petiles que calles de l'Hel. pomalia. (Panecri: Differenze anatomiche tra l'll. pusatia $e l \mathrm{H}$. Luculun; in strobs: Giornalo di Me. bacol., Anno 1: Pavia, 1853). 


\section{Melix Incorum.}

Helix huconum, Linne - System. Nalur. Edit. X ; 1758, 1, pag. 773.

- - Müller - Verm. Histor. II, 1774; pag. 46, - Firussac: Hist. Moll. pl. 21, A ; fig. 1-7.

R. P. - Plaine du Pò (nord): Vallée du Ticino (cette espèce est assez commune dans le Jardin botanique et en quelques autres horts de Pavie) - Plaine du Pó (sud): Val Coppa, Casteggio (Strobel).

Gen. BULIMUS. (a)

(Bulimus (emendat.), Scopoli - Introll. ad. hist. natur.; 1775.)

Sect. E N A. $(b)$

(Ena, Leach - Brit. Mull., pag. 112; (x Turtun 1839.)

\section{Bulimus montanus.}

Bulimus montanus, Draparnaud - Tubl. Moll. 1801 - et Hist. Moll. 1805; pl. IV. fig. 22 .

V. C. - Val de la Toce: Gondo, au Simplon, $780^{\mathrm{m}}$; dans les haies, les taillis et les forêts; après les pluies il monte sur les trones des arbres à épiderme lisse, sur les bois morts, ete. Terrains crystallin en genre (Stabile).

(a) L'absence du tard et les vésicules muqueuses; le flagellum plus ou moins latéral, et presque jamais terminal, en forme de massue ou ubové; une màchoire fnement strice et à peine crénelée sur les bords; les tentacules infíricurs proportionnellement plus courts, etc. sont les caractères qui distinguent nos Bulimus des llélices.

(b) Michoire striee, surtout vers le bord inferieur; haute, arquee (B. momtanss) ' "u citruite et lingerement subrustriforme (B. obscumus)- 


\section{Bulimus obscurus.}

Wielix obscura, Müler - Verm. Hist., 177/; 1I, pag. 103.

Bulimus obscurus, Drap. - Tabl. Moll., 1801; et Hist. Moll. 1805; pl, IV , fig. 23 - Mnquin-Tandon : Moll. de France ; 1855, pag. 292, pl. XXI, fig. 5-10 (Anatom.).

V. C. - Vallée de la Toce: Domo d'Ossola, 300m (Prada); rives du Verbano $210-500^{\mathrm{m}}$ (Stabile) - Val de la Stura de Lanzo: toute la vallée, $460-1600^{\mathrm{m}}$; région serpentineuse et gneissique (Stabile).

R. P. - Plaine du Pò (nord): Vercelli, $150^{\mathrm{m}}$ (Mella); Mezzana Corti, près la-Cava (Strobel) - Plaine sud: Saluzzo (Mella); Val Madonna, Alexandrie; Val Aversa, etc. (Strobel) 90-360 ${ }^{\mathrm{m}}$.

V. T. Zavatarello, Montalto en Val Trebbia et Val Tidone; Arquata en Val Scrivia (Strobel, Villa). Au pied des haies, sur les murs humides; dans les régions montagneuses et alpestres sous les grosses pierres.

\section{Sect. Z I I I A $(a)$}

(Zebrina, Held - in Isis; 1837, pag. 917.)

\section{Bulimus detritus.}

Helix delrita, Müller - Verm. Hist. 1774; II, pag. 101.

bulimus detritus, Studer - Kurz. Verzeichn; 1820, pag. 88 - Moquin-Tandon : Moll. de France; 1855, pag. 294, pl. XXI, fig. 24; pour l'Anatomie fig. 12-20.

B) sepinm . Gmelin - System. Natur. 1788, pag. 3654 - Obsolete et irregulariter fusoo-radiata (Moquin-Tandon: loco citato pl. XXI, fig. 21 et 22).

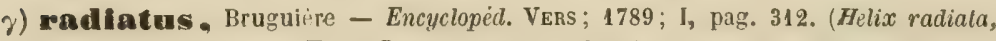
Férussac - Tabl. System. 1822 - Moquin-Tandon: loco citato, fig. 23.)

V. C. - Vallée de la Doire Bàltea: Aosta, $600^{\mathrm{m}}$ (Deponti) - V . de la Doire Riparia: Suse, Chaumont, $500-600^{\mathrm{m}}$ (Stabile, Strobel, Mortillet - Mutat, $\alpha$, et $\gamma$ ).

(a) Mâchoire peu arquie, failjtrment striée vers le bord inférieur et un peu rostriforme. 
V. T. - Val Maira: Dronéro, $600^{\mathrm{m}}$ (Mellaj - Val Slura de Ciu aeo: Rocea-Sparvéra, Vignólo, Gajola près Cunco, \$80-578m (Mort.)

Sect. C O I I I I.

(Chondrula, Reck - Ind. Moll.; 1837, pag. 87- Chondrus (partim) Cusier Rigne Anim. 1817, 11 .

\section{* 4. Bulimus tridens.}

Jelix tridens, Müller - Term. Hislor.; 1774; II, pag. 106.

Bulimus tridens, Brugniere - Encycloped., VERS, II, 1792, pag. 350.

Pupa tridens, Draparnaud - Tabl. des Moll. 1801: Nisl. Moll., 1805̃ ; pl. 3, figr. 57. - Moquin-Tandorı: Moll. de France, 1855; pag. 297; pl. XXI, fig. 25-30. (Anatom.); Ad. Schmilt - Geschlecht. der Stylommatoph. 1855; pl. X, figr. 71 (Anatom.).

R. P. Plaine du Pô (nord): Turin, $200^{\mathrm{m}}$ (Mortillet), Superga, $670^{\mathrm{m}}$ (Villa); Nezzana-Corti, Guasta, rives du Tessin, $80^{\mathrm{m}}$ (Strobel) - Plaine sud: Vaienza, Alexandrie, Stradella, 80-100m (Strobel); Dronéro, $600^{\mathrm{m}}$ (Mella).

V. T. - Val Tidone, et Val de la Trebbia: Montalto, Bobbio, etc. (Strobel).

\section{* 5. Bulimus quadridens.}

Jlelix quadridens, Muller - Yermium Hist. 1774, II, pag. 107.

Bulimus quadridens, Bruguière - Encycl, Vens. II, 1792 ; pag. 351.

P'upa quadridens, Draparnaud - Tabl. Moll. 1801; Hist. Moll. 1805. pt. 4, fig. 3 Moquis-Tandon: loeo cilato, pl. XXIl, fig. 3 (Anatum.)

a) normalis, mihi - Coquille de taille moyenne. Hahit. les régions infirieurs.

3) nana , mihi - Coquille plus petite, raccourcie. Hahit. Irs régions élevées, $(1 / 00-1800 \mathrm{~m})$.

V. C. - V. de la Toce: Gondo, Isella au Simplon, 600-700 région granitique; sous les pierres, dans les endroits rocailleux et

(a) Màchoire a bord infirieur un peu rostriforme; strice (B. tridens); ou presulue lisse (B. quadidens). 
parmi fes buissons (Stabile) - V. Stura de Lanzo: au dessus du village de Balme, Alpe de la Mussa, ete. $1600-1800^{\mathrm{m}}$; roches crystallines; sous les grosses pierres, avec l'H. ruderata, la Claus. alpi$n a$, etc. (Stabile) - V. de la Doire Riparia: Suse, $540^{\mathrm{m}}$ (Strobel, Stabile, Mortillet) région crystalline.

R. P. - Plaine du Pó, sud: collines de Superga, 670 ${ }^{\mathrm{m}}$ (Villa, Strobel); Sanctuaire de Crea; Rosignano, à sud-est de Casale, $220-400^{\mathrm{m}}$; collines de l'Astésan, 140-300m (Mella); Voghera (Strobel); Novi (Mortillet); vallée du Tanaro; Stradella (Strobel).

V. T. - Val Slura de Cuneo (ou de Demonte): Vignolo, Bæ $30^{\mathrm{m}}$; Rocea Sparvéra, $560^{\mathrm{m}}$ (Mortillet). Toutes ces localités sont près de Cuneo. Val de la Bòrmida: Acqui (Villa) - Val Scrivia: Serravalle, Corniasca (Mortillet) - Val Staffora; Val Coppa; Val Aversa; Val Tidone: Zavatarello; Val Trebbia: Bobbio (Strobel).

Sect. F I U S SA CIA. $(a)$

(Ferussacia, Risso - Hist. nat. de l'Europe mérid., 1826, IV.)

\section{* G. Pulimus subeylindrieus.}

Helix subcylindrica, Linné - System. Natur.; Édit. XII, 1767; non Monfagu.

- lubrica, Nüller - Verm. Histor. II; 1774, pag. 104.

Bulimus lubricus, Bruguière - Encyclopéd. méthod. Vers; I, 1789, pag. 311.

Achatina lubrica, Menke - Synops. Mullusc.; 1830 , pag. 29.

Columma lubrica, De Cristoforis et Jan - Catal. rer. natur., etc. 1832.

Ferussacia subcylindrica, Bourguignat - Aménilés Malacol, in: Revre et Magasit de Zool., par Guérin; 18 s.6.

V. C. - Vallée de la Doire Bảltea: collines de Viverone, (terr. erratique) $330^{\mathrm{m}}$ (Mella) - Val Stura de Lanzo: régions alpestres, $1400-1800^{\mathrm{m}}$; région gneissique et serpentineuse; sous les grosses pierres, près les ruisseaux, associé au Bul.quadridens, à l'H. glacialis, ruderata, etc. à la Pupa triplicata, etc.; un peu plus petite que les exemplaires vivants daus les régions des collines et de la 
plaine (Stabile) - V. le la Doire Riparia: plaine du Mont-Cenis, $1920^{\mathrm{m}}$ (Mortillet) - Rochemolles au Monte-Thabor, $1660^{\mathrm{m}}$ (Stabile) Terr. crystallin.

R. P. - Plainc du Pó, nord: Vercelli, $160^{\mathrm{m}}$ (Mella); Mezzana-

Corti en Lomellina $70^{\mathrm{m}}$ (Strobel); Turin, aval, $200^{\mathrm{m}}$ (Mortillet) Plaine sud: environs d'Alexandrie, 82-90 (Strobel, Mortillet).

F) pachygastra, mibi - Testa ventrosula, nilida, corneo-rufescenti.

R. P. - Ilaute vallée da Pó: Ghisole près Paesana, $840^{\mathrm{m}}$ (Mort.).

V. T. - Val Varàila: Brossasco près Venasca, $600^{\mathrm{m}}$ (Mort.).

\section{Gen. CACILIANELLA. $(a)$}

(Cocilianella, Bourguignat - Aménités Malacol, in: Recre el Magasin de Zool. par Guérin, août, 1856: (emendat. de Cecilioides, Férussac, teste Blainville, in: Dict. Sc. nat. T. VII, 1817, pag. 332.)

Bulimus (partim) - Achatina (partim) - Cionella (partim) - Columna (partim) - Polyphemus (partim) - Glandina (partim), quorund. auct.

Acicula, Risso - Hist. nat. Europ. mèrid.; Tom. IV, 1826; non Acicula, Hartmann.

Sira (partim), Adolphe Schmidt - Geschlechtsapparal der Slylommatophoren; 1855, pag. $42(b)$.

(a) Cacilia = caca. Mollusque aveugle, ou que I'on suppose aveugle. Les pédoncules oculigères we sont pas renflés à l'extrémité, comme chez les autres Bulimes; le bouton apical est presque nul, et à la place du globe oculaire existe une petite dépression anuulaire lisse. N. Férussac (Essai d'une méthod. conch., 1807, pag. 77) a observé l'animal avec une forte lentille et il n'a pu découvrir aucun indice de points oculaires. Nilsson (Hist. Moll. Suecia, ete., 1822, pag. 39) dit: "In hae specie (acicula) oculi sane nulli deteguntur, nigi alba sunt, uti ipsa tentacula.. M. ${ }^{\mathrm{r}}$ Baudon a trouvé une seule fois an individu vivant de $C$. acicula sous une pierre, derriere un mur humide. - L'animal, dit-il, est blanchâtre, demi-trasparent, un peu rosé à sa terminaison à cause de la présence des viscères; il n'y a pas apparence d'organe visuel au sommet des tentacules; cependant j'ai constaté que l'individu que j'avais sous les yeux était extrèmement sensible à la lumière; il est probable que l'impression de l'air lui est désagréable et qu'il cherche plutòt a l'íviter, étant toujours habitué à rester caché assez profondément. (Baudon: Nouv. Catal. Moll. de l'Oise; 1862). Quoi qu'il en soit l'absence, ou la singulière conformation des organes visuels chez ces petits mollusques, de même que leur habitat, sont des caractéres sufilssants pour les separer et placer dans un genre special.

(b) La C. acicula, Müller; la Stenogyra (Achat.) octona, Chemnitz; la Rumina (Bal.), decollala, Linn.; et quelques Achatinelles, entrent dans ce genre. Le principal caractère cunsiste dans la forme des dents ou napilles linguales; la dent centrale de chaque rangée est trés petite et carríe; (Adolphe Schmidt). 


\section{- Crecilianella acieula.}

Buccinum acicula, Müller - Verm. Hist., II, 1774, pag. 150.

Cionella acicula (partim), Jeffreys - System. test., in: Trans. Linn., 1830, 1. XV1, 2. partie, pag. 347.

Achatina acicula, Rossmässler - Icon. d. Land und Süsswass. Moll., 1939, Heft. IX et $\mathrm{X}$, fig. 658 .

- aciculoides, De Betta - Malacol. della valle di Non (Tirolo ital.), Parte I, Moll. terr., 1852, pag. 75, fig. 3 ; non aciculoides, Jan.

Bulimus (sect. Acicula) acicula, Moq.-Tandon - Hist. Moll. de France, 1855, p. 309, pl. XXII, fig. $32-34$.

Crecilianella acicula, Bourgnignat - loco citato, 1856, pag. 215, pl. 18, fig. 1.3 (tirage à part).

Testa parva, non umbilicata, elongata, fusiformi-cylindrico-acicularis, non inflata, gracilis, diaphana, polita, albida. Spira sensim altenuata, apice obtuso. Anfractus 6 convexiusculi, satis rapide crescentes, sutura vix marginata distincti; ultimo non inflato, $1 / 3$ longitudinis requante. Apertura paululum obliqua, oblonga, sursum strictiuscula. Peristoma non continuum, simplex, acutum ; margine externo antrorsum vix producto, marginibus callo tenui junctis; columella arcuata, mediocriter truncata, ad basim aperturæ fere attingente.

Coquille dextre, petite, allongée, fusiforme-cylindrico-aciculaire, non ventrue, grêle, lisse, hyaline, blanchàtre; fente ombilicale nulle. Spire composée de six tours un peu convexes, croissant assez rapidement; sommet obtus; suture à peine marginée; dernier tour non enflé, surpassant à peine le tiers de la hauteur totale de la coquille. Ouverture sub-oblique, oblongue, un peu étroite. Péristome non continu, simple, aigu; bords marginaux réunis par une callosité plus ou moins distincte; bord droit, ou externe, très peu arqué en avant; columelle médiocrement arquée, tronquée, atteignant à peine la base de l'ouverture.

Hauteur: 5 millim. - Diamètre: $1 \%$ millim. Ouverture: Haut. 2 - Diam: $05 / 6$ millim.

R. P. - Plaine du Pô, nord: Turin, alluvions (Mortillet); Mezzana Corti près de la Cava, Lomelline, (Strobel) - Plaine sud: Alexan- 
drie (Slrobei) - Collines transpadanes: Sciolze, à l'est de Turin (Mclla).

Obs. Les quelques individus recueillis près de Turin ont les bords marginaux du péristome réunis par une callosité bien distincte, et qui sur quelques individus plus adultes présente sur le milieu de l'avant-dernier tour à l'entrée de l'ouverture, un'éminence luberculeuse. Celte pièce ecpendant, de même que le pli ventral de la Balia perversa, et de quelques Pupes, n'est pas un caractere important pour la détermination des espèces. La nature du sol sur lequel le mollusque vit, l'àge mème dı mollusque, les conditions accidentelles, ete. exereent une diverse intluence sur le développement de lanimal et par conséquent sur la coquille; ces pières accessoires bien prononcées sur certains individus, sur quelques autres au contraire, et dans la mème localité, sont suuvent peu apparents, ou nulles. Inutil de faire observer que les coquilles des Cacilianelles, ainsi que celles des Carychies, etc. après la mort de l'animal deviennent opaques et d'un blanc de calcaire.

\section{Crecilianella aciculoides.}

Columna aciculoides, Jan - Mantissa in secundan partem Catal. Testac. cxtanl. in coll. De Crist. et Jan, etc.; 1832, pag. 2.

Polyphemus aciculoides, Villa - Disposil. system. Conch.; 1841, pag. 20 ; evilus. synonym.

Achatina aciculoides, L. Pfeiffer - Monogr. Helic. viv., II, 1848.

- acicula, De Betla - loco cilato, pag. 74, fig. 2 ; non acicula, Müller.

- Jani, De Bella e diartinati - Cat. Holl. delle Provincie Venele; 1855, p. 59. Cacilianella aciculoides, Bourguignat - Aménités malacol. etc. jantier, 1857, pl. 1, Cilandina veneta, Charpentier - in Strobel: Essai d'une distribut. orogr. giogr. des Moll. terr. dans lı Lombardic: in: Mém. Acad. des sc. de Turin, 1857.

- Jani, De Betta - Esame critico intorno a tre molluschi del genere Glandinu; in: Atti dell' Istituto Veneto, 186t, pag. 23, lïr. 4-6. (lirage a part).

Testa parva, non umbilicata, fusiformis, polita, diaphana, albidia. Spira turrito-attenuata, apice acutiusculo. Anfractus 6 convexiusculi, sutura marginata, sub-duplicata distincti; ultimo magno, ventricosulo, dimidium longitudinis sub-eqquante. Ipertura satis obliqua, elongato-piriformis, basi sub-rotundata, superne coarctata. Peristoma 
non continuum, simplex, rectum, acutum, marginibus callo tenuß junctis; margine dextro antrorsum producto; columella sub-arcuata, abrupte truncata, vix ad basim aperturx altingente.

Coquille dextre, petile, fusiforme, lisse, byaline, blanchàtre; fente ombilicale nulle. Spire de six tours médiocrement convexes, sommet peu obtus; suture assez largement marginée, entourée inférieurement d'une rainure imitant une seconde suture lineaire; dernier tour assez ventru, égalant presque la moitié de la longueur totale de la coquille. Ouverture oblique, piriforme-allongée, sub-arrondie à la base, rétrécie à sa partie supérieure. Peristome non cuntinu, simple, aigu; bords marginaux réunis par une callosilé; bord droit ou extéricur arqué en avant; columelle médiocrement arquée, fortement tronquée, atteignant à peine la base de l'ouverture.

Haut. 6 à $6 \frac{1}{2}$ millim. - Diam. 2 à $21 / 6$ millim. Ouverture: haut. $2{ }^{5} / 6 ;-$ Diam. 2 millim.

R. P. - Vallée du Ticino, à la plaine (Strobel) - Vercelli, charriée par la Sésia (Mella). C'est l'espèce que l'on trouve plus communement en Lombardje (Legnano, Lecco), à Lugano, etc., ordinairement au pied des vieux murs mème des maisons, à une profondité de 20 à 30 centimètres, d'où elle est exportée par les fourmis, probablement après en avoir dévoré l'animal. Il paraît cependant que ces mollusques ne demeurent pas exclusivement sous terre; la $C$. aciculoides, a été recueillie vivante par M. ${ }^{r}$ le D. ${ }^{r}$ Martinati de Vérone sous les débris entassés d'un vieux mur écroulé; moi aussi j'ai trouvé dans les environs de Legnano une Cæcilianelle (qui n'est pas ni l'acicula, ni l'aciculoides) dans le petites cavités naturelles d'un bloc de poudingue. M. ${ }^{r}$ Pirovano, peintre et naturaliste de Legnano, m'a communiqué des beaux exemplaires de C. aciculoides, trouvés déjà pour la deuxième fois au pied de la muraille d'una maison, et exportés par les fourmis; quelques-uns de ces exemplaires atteignent jusqu'à 7 millim. de hauteur. Chez les individus peu adultes le dernier tour égale la moitié de la hauteur totale de la coquille, mais ceux qui sont arrivés à leur entier déve- 
loppement (haut. $6 \frac{1}{2}$ a 7 millim.) ont le dernier tour un peu plus court que la moitic de la spire.

Obs. Il y a mème chez les coquilles certaines espèces qui paraissent, pour ainsi dire, destinées à embrouiller par leurs noms la science, et à engendrer la confusion parmi les naturalistes qui, à leur tour, pour s'en débarrasser, font servir les mêmes noms comme de passe-ports scientifiques aux diverses espèces que un faux air de ressemblance souvent confonde, quoique en effet elles sont bien distinctes. Sont de ce nombre infortuné l'II. striata, l'H. planospira, la Cacil. acicula, et bien d'autres encore. D'ici les opinions les plus disparates, les deseriptions si peu correlatives, les caractires les plus opposés attribués souvent à une même espèce, selon qu'on a cru la réconnaitre dans l'une ou dans l'autre des différentes formes de coquilles. Je ferai quelques rémarques sur les Crecil. acicula et aciculoides. Les und régardent, et avec raison, comme vraie aciculoides Jan, l'espèce fusiforme, ả dernier tour assez ventru, égalant la moitié de l'hauteur totale de la coquille, à columelle saillante, tronquée, etc.; c'est notre aciculoides (que nous désignerons ici par la lettre A). Pour quelques autres, au contraire, et parmi eux M.' De Betla $(a)$, ce serait celte mème forme la vraie acicula, Müller; et le prof. Jan aurait nommé aciculoides l'autre espèce grêle, allongée, à dernier tour non renflé et surpassant un peu le tiers de la longueur totale de la spire, à ouverture plus courtement ovale, à columelle à peine tronqueé, etc.; c'est notre acicula (que nous distinguerons par la lettre B). M. ${ }^{r}$ Strobel (b), et dernièrement M. ${ }^{r}$ De Betta $(c)$ donnent le nom de aciculoides, Jan, comme double emploi de acicula, Müller; la forme A restant donc sans nom, $\mathbf{M}_{0}{ }^{\mathrm{r}}$ de Charpentier, qui suivait l'opinion de M.' Strobel, l'avait nommée veneta. Quant à MII. ${ }^{\text {rs }}$ Küster, L. Pfeiffer, et peut-être même Moquin-Tandon, pour eux notre espèce A serait identique avec l'Achat. Hohenwarti, Ros-

(a) De Bclla: Malacologia terr. e Auv. delle Valle di Non, nel Tirolo italiano: Parte I, Moll. terrestri; Verona, 1852. - De Betla e Martinali: Catal. Moll. delle Provincie Venete; Veruna, 1855.

(b) Strobel - Essai d'une distrib. orogr., elc. Ioro citalo.

(c) De Betla - Esame crilico, etc. loco citato. 
smässler. M.' De Bella nous assure que ses Achat. acicula el aciculoides ont été determinées par $\mathbf{M}^{\mathrm{r}}{ }^{\mathrm{r}}$ le prof. Jan mème; la raison serait donc du côté de M. ${ }^{\mathrm{r}}$ De Betla; mais il est à savoir s'il n'est pas possible que M. ${ }^{5}$ le prof. Jan, qui depuis long temps ne s'occupe plus de malacologie, déterminant une petite coquille baptisée par lui un tiers de siècle avant, il ne se soit pas trompé, et n'ait pas confondu, chose très facile aux conchyliologistes même les plus exercés, les caractères d'une espèce avec ceux de l'autre; et d'autant plus je suis porté à le croire, parceque ayant en 1856 demandé à l'obligeance de M. ${ }^{\mathrm{r}}$ le prof. Cornalia, au Musée de Milan, quelques types des deux espèces en question, il me remettait comme type de l'aciculuides, Jan, la forme A.

Le mieux donc que l'on puisse faire e'est de considérer l'espèce A conme la vraie aciculoides, Jan, et la forme B comme la vraie acicula, Nüller, et cela par plusieures autres bonnes raisons: MM. rs Rossmässler, Charpentier, Küster, L. Pfeiffer, et presque tous les conchyliologistes allemands et français, ne doutent nullement que l'espèce B est la $C$. acicula, Müller; c'est toujours cette forme qu'ils décrivent, qu'ils figurent assez fidèlement, et qu'ils envoyent. La $C$. Hohenwarti, Rossm. est une espèce différente des toutes les autres et que l'on ne doit pas confondre avec l'aciculoides; nous n'en parlerons donc pas davantage. Quant à la $C$. aciculoides, les exemplaires qui ont servi à M. ${ }^{r}$ Bourguignat pour sa monographie, lui ont été rémis par M. ${ }^{\mathrm{r}}$ Fischer déjà déterminés, et la $C$. aciculoides de Bourguignat est bien notre aciculoides ou la forme A. La collection des MM.rs les frères Villa de Milan est une des plus riches et en même temps des plus anciennes; ces messieurs étaient amis et toujours en correspondance d'échanges avec MH. ${ }^{r s}$ De Cristoforis et Jan, il est donc indubitable qu'ils auront eu de l'obligeance des leurs amis et collègues ou les types mêmes de l'espèce de M. ${ }^{\mathrm{r}} \mathrm{Jan}$, ou pour le moins la determination des leurs exemplaires; or la $C$. aciculoides des MM.rs Villa est identique à celle que j'ai récue de notre Musée, et à celle décrite et figuré par M. ${ }^{r}$ Bourguignat. Enfin, ce qui est très important, la forme A est presque exclusive des nos pays, c'est à dire de l'ltalie boréale, tandis que l'espèce B habite la France, 
P'Allemagne et plusieures autres contrees d'Europe; comment dons. pourra-t-on raisonnablement supposer que Mïller n'ait pas eu connaissance de l'espèce plus cosmopolite, et ail imposé le nom de aciculı à une espèce étrangère à sons pays? Hais ce qui est un peu à déplorer c'est qu'on a souvent usurpé le nom de aciculı pour désigner plusieurs espèces de Cæcilianelles bien distinctes. Nous dirons seulement en passant, qu'en Lombardie la $C$. aciculı est quelquefois représentée par une autre forme un peu moins allongée, à dernier tour plus grand, mais non enflé, à ouverture oblonge, assez étroite, à columelle presque droite, à bords marginaux du péristome réunis par une faible callosité présentant quelquefois vers le milieu de l'avant-dernier tour, à l'entrée de l'ouverture, une petite éminence luberculeuse; c'est une espèce différente même de la Liesvillei, Bourguignat.

Les espèces du genre Cacilianella, mal connues jusqu'à nos jours, ont été diligemment étudiées par M. ${ }^{\mathrm{r}}$ Bourguignat; il a donné les diagnoses et les figures de bien 20 espèces vivantes et d'une fussile $(a)$. Quelqu'une de ces espèces ne serait-elle pas un peu questionnable?...

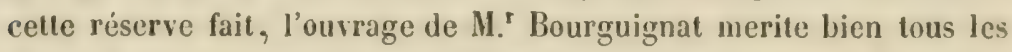
éloges à son infatigable auteur! D'autre cơté nous oserions demander à M. ${ }^{r}$ De Betta (naturaliste d'ailleurs très éstimable) s'il n'ait pas un peu trop impitoyablement condamné une quelque bonne espèce de Cocilianella à servir de synonyme à son acicula; et s'il n'est pas probable que dans l'un ou dans l'autre des nombreux et si divers pays d'Europe cités par lui, la $C$. acicula soit plutùt représentée par une quelque autre espèce bien différente!. Ainsi, par exemple, nous avons reçu de l'obligeance de M. ${ }^{r}$ Gaudin de Lausanne, et de M. ${ }^{r}$ Arthur Issel de Gènes deux Cixcilianelles, recueillies l'une par M. 'Gaudin méme près de Palerme, l'autre des environs de Gènes, lesquelles ont rien affaire avec l'acicula Müller!

Quant à la détermination générique des ces jolies petites coquilles, il n'y en a pas d'autres, peut-être, qui aient rẹ̣u des conchyliolo-

(a) d. R. Bourguignat: Aménilés Malacologiques; in Recue el Mag. de Zool., par Guc. rin, 1856, et janvier 1857 - Le mime. Malacol. terr. et Rue. de lie Brelagne, Paris, 1860 ; - Llude synonymique sur les Moll. des Alpes maril. publiés pur Risso en 1802; Paris, 1861: - Malacologic l'Ai2-les-Bains; Paris, 1864. 
gístes tant de noms différents! Les uns les ont réunies sans distirction tantôt à l'un, tantòt à l'autre des divers genres; les autres en ont fait des simples sections; quelques-uns enfin ont établi pour elles des genres ou sous-genres speciaux. M. ${ }^{\mathrm{r}}$ de Férussac avait déjà créé le genre Cecilioides (a) (corrigé ensuite par M. ${ }^{\mathrm{P}}$ Bourguignat en Cocilianella); plus tard il réunissait ces petites coquilles, à titre de simple section, au genre Helix; enfin M. ${ }^{{ }}$Risso créa le genre Acicula, mais ce nom on ne peut pas l'adopter, soit à cause de l'antériorité de celui de Férussac, soit pour eviter toute confusion par la préexistence d'une Acicula Hartmann, double emploi de Acme, Hartmann, mollusque terrestre, operculé. Ainsi les Cacilianelles rapportées premièrement par Müller au genre Buccinum, ont reçu ensuite les denominations de Helix, Cecilioides, Cochlicopu, Achatina, Acicula, Cionella, Colunna, Styloides, Polyphemus, Glandina, etc. Nous n'entrerons pas ici en discussion sur la valeur scientifique de toutes ces appellations, nous ferons seulement obsérver que, à l'état actuel de la science malacologique, des qu'on vient de découvrir des nombreuses et importantes différences anatomiques chez les mollusques qui avaient été jusqu’à present, par la simple analogie de formes de leurs coquilles, plus ou moins improprement rapportés à des genres différents, les noms de Helix, Bulimus, Achatina, etc. ne peuvent servir que comme des noms collectifs et conventionnels; pas pour désigner scientifiquement un group d'espèces bien caractérisé et distinct. Au pis aller, quand même ou voudrait réunir les Crecilianelles à l'un ou à l'autre des genres connus, ce n'est pas certes du genre Glandina qu'on doit les rapprocher; on sait que les vraies Glandines sont prives de mâchoire, et leur langue est organisée comme celle des Testacelles et des Daudebardies; tandis que les Cacilianelles (au moins l'acicula) sont pourvues de mâchoire, et leurs denticulations linguales sont très semblables, par la forme et l'arrangement, à celles de Rumina (Bulimus) decollala, et de Stenogyra (Achatina) octona. Quelques conchyliologistes réunissent les Cacilianelles à la Glandina algira; en effet, d'après la forme de 
la coquille, on dirait que les Cxecilianelles sont des Glandines algires en miniature; mais la Gl. algira est une vraie Glandina, tandis que les Crecilianelles ne le sont pas.

\section{Gen. CLAUSILIA.}

(Clausilia, Draparnaud - Hist. Moll. ; 1805, pag. 24.)

Sect. C H A IT P E T I E I I A, mihi. (a)

(Clausiliastra (partim), L. Pfeiffer - Versuch einer Anordnung des Heliceen nuch natür. Gruppen; in: Malakiozool. Blätter; II, 1855.)

\section{Clausilia diodon.}

Clausilia diodon, Studer - System. Verzeichn. etc. 1820; Charpentier: Cal. Mull. Suiss., 1837 ; pag. 17, pl. 2, fig. 8; L. Pfeiffer: Monogr. Helic. vivent. Vol. II, 1848, pag. 402; (non $\mathrm{Cl}$. diodon, quorund, auctor. et collectionum $=\mathrm{Cl}$. commulata, Rossm., de l'lllyrie, Carniole, etc.)

\footnotetext{
V. C. - Région inférieure du Simplon : Isella, Gondo, $600-710^{\mathrm{m}}$; terrain granitique (Stabile, Charpentier, Venetz).
}

(a) Coquille lisse, ou presque lisse; d'un corné uniforme, pâle; à suture non papillifere, ou avec quelques rares papilles et peu apparentes. Clausilium non échancré. Lunelle nulle. Plis palataux ordinairement au nombre de 2 , Junt l'inférieur trés cuurt. Lamelle spirale désunie, c'est à dire ne suivant pas la mème direction de la lamelle supérieur, mais aboutissant entre celle-ci et la suture. (Inutile de dire qu'on observe les Clausilies, les Pupes, etc. la pointe en haut, l'ouverture, soit la tête, en bas!)

Mâchoire à stries verticales faibles, un peu rostriforme au milieu.

La section Clausiliastra de M. ${ }^{r}$ L. Pfeiffer, comprend les Clausilies à lunelle nulle, à coquille lisse, à clausilium échancré. Les espèces du Piémont appartenantes à co group ont le Clausilium entier; nous ne puuvons pas donc adopter qu'en partie le nom de M.r Pfeiffer. Et tant moins admettre le nom de Marpessa, Gray, parceque il paraît bien que cet auteur ait créé celte division pour les seules Clausilies a Clausilium échancré (Voyez: Moquin-Taudon, Moll. de France, 1855; 11, pag. $316-\mathrm{Et}$ Mlartens, in Albers: Die Heliceen, etc. 2. édit 1860. Clausilia, Sect. Marpessa: a Plicue palatales plerumque quatuor; et clausilium emarginatum. •)

C'est à feu M.r de Charpentier qu'on doit une division des Clausilies en sections uu groupements naturels. (Essai d'une classification nuturelle des Clausilies; in: Journ. Conch., par Petit de la Saussaie; Paris, 1852.) Quvique quelqu'une de ses 16 sections démande à ètre mudifté, le travail du savant malacolugiste n'est pas moins précieux pour la science 1 
Vil dans les fentes des murailles qui soutiennent çà et là la grande roule, ou bien enfoncé parmi les debris de rochers entassés, et les racines entrelacées des buissons, d'où il sort après la pluie, se promenant même sur les dés de granite dont est pavé le bord du grand chemin, et régagnant bientôt ses rétraites. Les mêmes mœurs sont ceux de la Claus. Thomasiana, et des ses variétés.

\section{Clausilia Thomasiana.}

Clausilia Thomasiana, Charpentier - in Küster: Cinchyl. Cabin. pag. 48, pl. 5 , fig. 10-13; - L. Pfeiffer: Monogr. Helic. viv. Vol. III, 1853 pag. 600.

Testa anguste et breviter rimata, fusiformis, striata, solidiuscula, nitidula, sub-pellucida, corneo-virescens, vel corneo-rufescens (epidermide sæpe decidua). Spira sensim attenuata, apice ubluso. Anfractus 10-11 convexiusculi, sutura mediocri distincti; ullimus antice non inflatus, pone rimam obsolete compresso-gibbus. Apertura ovalipiriformis, strictiuscula; sinulo subrotundo. Lamella supera tenuis, marginem aperturæ altingens; infera mediocris, humilis, parum flexuosa, introrsum sub-bifida; spatium interlamellare læve; lamella spiralis, a supera, disjuncta, inter hanc et suturam progressa. Plicæ palatales 2, una supera, prope suturam, longa, tenuis; altera vero brevissima, postica; subcolumellaris emersa. Lunella nulla. Peristoma vix continuum, reflexum, marginibus callo tenui junctis.

Coquille fusiforme, un peu solide, pourvue d'une fente ombilicale, petite, étroite; test un peu luisant, demi-trasparent, à rides longitudinales obliques, assez marquées; corné-rougeâtre, et quelquefois à épiderme un peu verdâtre. (II arrive souvent d'en trouver de vivants qui ont déjà perdu, en tout, ou en partie, leur épiderme). Spire composée de 10 à 11 tours un peu convexes, sommet obtus; suture médiocre; dernier tour offrant à la base, près de la fente ombilicale, une compression gibbeuse, très courte et très peu apparente. Ouverture ovale-piriforme un peu étroite; elle parait conme légèrement subanguleuse près du pli subcolumellaire; goutlière peu haute, presque arrondie. Lamelle supérieure mince, avancée; l'inférieure 
médiocre, un peu écartée de la supérieure, buwble, sub-bilido en dedans; lamelle spirale assez avancée, el passant à côté de la lamelle supérieure, entre celle-ci et la sulure. Plis interlamellaires nuls; palataux 2, opposés à l'ouverture (l'un supérieur, près la sulure, assez long; l'autre très court, placé en arrière); pli subcolumellaire émergé. Lunelle nulle. Péristome non continu, réfléchi, à bords marginaux réunis par une faible callosité.

\section{Hateur: 13 a 16 mill.; Diametre. 3 mill.}

Clausilium. Pédicule, long de 1 mill.; lame, long. 2 mill.; oblongue, étroite, mince, blanchàtre-nacrée, un peu arquée, sub-arrondie à la base, sans échancrure, ni lobes; un peu rétrécie en baute, à l'insertion du pédicule.

a) Thomasiana, Charp. - Testa minore, ventrosiuscula, epidermide luteo-viridescenti obductu.

8) Verloanensis, mlhi - in Strobel: Essai d'une distribut. orogr..géogr. des Moll terr. Lomb.; In: Mém. Acad. de Turin, 1857, pag. 23 (sans descript.) - Stabile abbé Juseph: Descript. de quelques Coq. nouv., ou peu connues; in: Revue et Mag. Zool. par Guirin; Paris 1859, N. 7 (iigurée) - Tesla cylindraceo-fusiformi, cornea vel corneo-rufescenti.

g) Bellardia, milli - Descript. etc. loco citato (figur.) - Testa sape majori, solidiuscula, inferne vix striatula; apertura basi rotundiori, peristomate Bub-incrassalo.

a) monticola, mihi - Descript. etc. loco citato (flgur.) - Testa ut in pracedenti, plica vero subcolumellari vix emersa; sutura, in anfract. superioribus, papillis raris, punctiformibus, irregulariter ornata. Assez rare 1

V. C. - Rives occidentales, inférieures du lac Majeur, $230^{\mathrm{m}} ; m u$ -

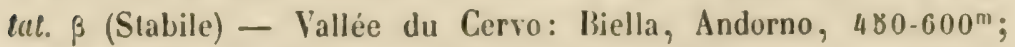
mulat. $\gamma$ (Casati, Mella) - Val de l'Orco: Castellamonte, 350 mulat. a (Emanuel Thomas) - Val Stura de Lanzo: Ceres, Chialamberto, Ala, 710-1090 ; mutat. $\beta$ (Stabile) - Viù, 770 ; mulat. $\gamma$, et ô (Bellardi).

Vit sous les pierres (crystallines), et entremélée aux racines des 
buissons, dans les petits vallons abrités, ou dans les vieux nurs sur les sentiers montagneux, d'où il ne sort qu'après et pendant les fortes pluies, pour regagner bientùt sa rétraite.

Obs. C'est en 18 въ que, visitant les côtés occidentales inférieures du lac Majeur, je découvrais cette nouvelle forme de Clausilie que, pour le moment, je nommais Verbanensis. Je ne ignorais pas cependant que une espèce nouvelle avait été trouvée à Castellamonte à l'entrée de la vallée de l'Orco, par feu le bolaniste Emanuel Thomas de Béx (Canton de Vaud, Suisse); et, à vrai dire, j'avais vu même cetle espèce (Claus. Thomasiana, Charp.) dans la riche collection de feu N. ${ }^{\mathrm{r}}$ de Charpentier, lorsque en 1834 j'ai demeuré quelque temps à Béx chez ce bon ami et illustre savant; mais je ne me rappellais plus les caractères de cette Clausilie. Il pouvait bien être donc que ma Claus. Verbanensis ne fùt d'autre chose que la Claus. Thomasiana!, quoique la distance qui les separe ne soit pas moins de $86 \mathrm{kilom}$, et dans une direction tout à fait irrégulière. J'envoyais done quelques exemplaires de ma Clausilie à $M^{r}{ }^{r}$ de Charpentier, mais ils lui arrivaient qu'il était malade ... dernière maladie qui l'enleva en peu de jours aux amis et à la science! Ayant ensuite rémis quelques individus de cetle espèce nouvelle à l'un et à l'autre des nos malacologistes, tous sont tombés d'accord en me félicitant de ma jolie et tout à fait nouvelle espèce de Clausilie!... Et ils n'avaient pas le tort, eux! Personne, à ce qu'il paraît, si l'on excepte, peut-être, M. ${ }^{\top}$ Küster, possedait la Claus. Thomasiana, et d'autant plus je me confirme dans celte opinion, parceque I. $^{r}$ de Charpentier, qui y tenait beaucoup à posseder plusieurs exemplaires de chaque espèce dans ses tiroirs, n'avait de celle espèce que trois seuls échantillons! Quant à consulter l'ouvrage de Küster, je défie les plus habiles des conchyliologistes à déterminer une espèce nouvelle de Clausilie d'après une diagnose abrégée et une figure petite et peu signifiante! Plusicures circonstances m'empèchaient alors, et même plus tard, de fair un vọage jusqu’à Lausanne, au Nusée de laquelle ville, $\mathbf{M}^{\mathrm{r}}{ }^{\mathrm{d}}$ de Charpentier avait legué, et y avait été déjà déposée sa collection de coquilles terrestres et fluviatiles, et son riche herbaire. Sur ces faits j'ai publié donc en 1839 (dans la Revue 
el Magas. de Zool. par Guérin, à Paris) la description de ma Claus. Verbanensis, et d'autrés espèces, elc.; et je l'enroyais à mes correspondants. Mais à present devant publier ce petit ouvrage, je me suis décidé, dans l'intérêt de la science, de faire une course jusqu’à Lausanne pour vérifier enfin la chose; et ce ne fut que merci à l'extrème obligeance de .1. 'r le Dirccteur llu Husce, le doct.' De La Ilarpe, que j'ai pu observer ce que je souhaitais de voir; car les collections de M. de Charpenticr sont enfermées dans une salle particulière du Nusée, et personne ne peut y tuncher excepté le Directeur, et celui-ci élait alors Irès indisposé. Les C'laus. Verbanensis et Thomasiana ne sont done que une scule espèce avec de légères modifications.

\section{Clausilia alpina.}

Clansilia alpina, mihi - in Strobel: Essai, ctc. loeo cititu, 1857: (sans descript.)

- olicucea? ?, Parreyss - flde Adulphe Schmidt (a). Non $\mathrm{Cl}$. olivacea, Cantraine.

- alpina, mili - Descript. de quelgues Cuq. ctc. loco supra cit., 1859. (nggur.)

Testa angusle et breviter rimata, ventriculoso-fusiformis, temuiuscula, pellucida, nitida, cornea, sursum subtiliter striala, infra medium striatula, læviuscula. Spira regulariter attenuata, apice obtusiusculo. Anfractus 11 convexiusculi, sutura mediocri, minute papillifera distincti; ultimus basi tumidulus, pone rimam obsoletc cristato-gibbus. Apertura ovato-pirifornis, basi sub-rotundata; sinulo parvo, subovato-rotundo. Lamella supera minuta, tenuis; infera humilis, remota, immersa, sape introsum sub-bifida; spatium interlamellare lave; lamclla spiralis disjuncta, nempe inter suturam et lamellam superam progressa. Plicæ palatales 2, (una supera satis longa; altera vero brevissima, postica); subeolumellaris emersa. I.unella

ia; M. Parresss dil que sa Claus. vlicacer prosient te la Suisse!; et je trouse dans louvrage de Chrorpentier: Essui d'une classifuculim nalur. de's Clausilies (in l'elit de la Saussaie: Jounn. Couch. WV, 1852.) X. 29, Claus, cerata Rossm.; $\beta$, minor, tenuior, callo parietali ohsoleto $=$ Cluus, olivacea, Parreyss; in schedis - Albania (Par. reyss: !?! 
unlla, ant rulimentalis. Peristoma non contiuum, appressun, reflexiusculum, marginibus remotis, callo tenui junctis.

Coquille fusiforme, un peu ventrue, un peu mince, pourvue d'une fente ombilicale courte et étroite. Test un peu luisant, transparent, de couleur cornée, un peu strié vers le sonmet, à rides inégales et presque éffacées au-dessous du milieu. Spire composée de onze tours peu convexes à suture médiocre et presque toujours ornée de pelites papilles; le dernier tour un peu ruguleux sur le devant, ayant à la base, près de la fente ombilicale, une compression gibbeuse peu saillante; sommet de la spire un peu obtuse. Ouverture ovale-piriforme, paraissant quelquefois subanguleuse près du pli subcolumellaire; goultière pelite arrondie-ovale. Lamelle supérieure petite, mince; l'inférieure à peine médiocre, profonde, non élevée, immergée, quelquefois sub-bifide en dedans; lamelle spirale un peu avancée entre la lam. supérieure et la suture. Plis interlamellaires nuls; palataux 2, opposés à l'ouverture (le supérieur, près la suture, assez long; l'inférieur très-court, placé en arrière). Ltinelle nulle, ou rudimentale, formée par une callosité très-légère, blanchâtre, allongée, droile, située au-dessous du plus court des plix palataux. Péristone non conlinu, un peu réfléchi, peu épais: bords marginaux un peu écartés, réunis par une faible callosité.

$$
\text { Hatutcur, } 15 \text { mill. - Diam. } 31 / 2 \text { à } 4 \text {, mill. }
$$

Clausilium comme celui de la Claus. Verbanensis; cependant le pedicule est quelque petit peu plus allongé, et la lame à peine un peu plus étroite.

V. C. - Les régions alpines de la Vallée d'Ala, division supérieure de la Val Stura de Lanzo, au-dessus de $1600^{\mathrm{m}}$ (Stabile). Vit sous les grosses pierres (crystallines), près les neiges et les ruisseaux; aves: la Vitrina pellucida, Müller - Vitr. annularis, Vénétz Zonites Petronelle, Charp. - Hel. ruderata, Studer - Hel. glacialis, Thomas - Pupa triplicata, Studer, etc.

Obs. La Claus. alpina a beaucoup de ressemblance avec la Claus. diodon, Studer, du Simplon; mais on distinguera aisément notre 
espèce à sa taille constanment plus grande, à son péristome plus comprimé, à son pli columellaire émergée, à ses sutures ornées, presque toujours, de petites papilles, elc.

Ces cinque formes de Clausilies (Thomasiana, Verbanensis, Bellardii, monticola et alpina) rigoureusement considérées, ne formeraient que une seule espèce diversement modifiée par l'influence des localités, du climat, ele. Ainsi, comme on peut le voir, dans les régions élevées la coquille devient lisse et moins solide, les lamelles s'amincissent, la suture s'orne de papilles, etc. mais si l'on fait une comparaison entre les deux formes extrènies, on voit qu'on ne peut les confondre tout à fait ensemble!

Sect. D E I. I A. $(a)$

(Delima, Hartmann - Erd und Süsswass. Gasteropod. 1840-44 - Papillina (partim), Moq. Tandon - Moll. de France, 1855.)

\section{Clausilia albogutfulata.}

Clausilia alboguttulata, Wagner - in Chemnitz: Neue System. Conch. Cabinet, XII; 1829, part. I, pag. 191. (Non Claus. alboguttulata, PIr. = Claus. or nata, Z., espéce bien difrérente!)

*) albopustulata, De Cristoforis et dan - Mantissa, etc. $1832=$ Claus. alboguttul., Wagner; var. longobardica, L. Pfeiffer - Symbol. ad hist. Helic. $1844-18 \div 6$.

V. C. - Cotes du lac Majeur, 208.280m (Stabile). Sur les murs et les trones des arbres.

* B) punetata, Michaud - Complément a Draparnaud, 1831; pag. 55, pl. XV, fig. 23 - Moquin-Tandon: Hist. nat. Moll. de France, 1855, pag. 326, pl. XXIII, flg. 31-37; et pl. XXIV, fig. 1-7. (Anatom.).

V. T. - Versant piémontais de l'Apennin; Val-Tidone: Zavata-

(a) Coquille lisse, de couleur corné-rougeảtre, un peu luisante, souvent ornée de papilles aux sutures. Lunelle entière; pli palataux (ordinairement un seul); lamelle spirale désunie. Clausilium non échancré.

Măchoire presque lisse, obtusement sub-rnstriforme au milieu du hord inférleur. 
rello; Val-Trehbia: Bobbio (Strobel); Val-Scrivia: Serravalle près Novi (Mella).

\section{Sect. I. A C I I I I I A. (a)}

Laciniaria, llartmann - Erd und Süssw. Gasterop., 1840-4t - Elia H. et A. Adams - The genera of recent. Moll., arranged according to their organization; Part. XXI, april, 1855; - Ipligena (partim), Moquin-Tandon: Moll. de France, 1855.)

\section{* 5. Clausilia plicata.}

Pupa plicata, Draparnaud - Tabl. des Moll. 1801; pag. 63.

Claus. plicata, Drap. - Hist. Moll. 1805; pl. IV , fig. 15-16; Rossmässler - Icon.d. Land-und Süsswass. Moll. 1835, I, pag. 78, fig. 31; Moquin-Tandon Hist. Moll. de France, 1855; pag. 338, pl. XXIV, fig. 13-16; Adolphe Schmidt - Geschl. der Stylomm., etc. 1855 ; pl. XII; 1lg. 97. (Anatom.)

V. C. - Alentours de Canobbio, au lac Majeur, 210-240m (Villa) Sort assez abbondante sur les murailles pendant la pluie.

Anomalia) Bistoma - Ore allero in anfractu ultimo instructa.

Trouvée dans la même localité par M. ${ }^{\mathrm{r}}$ Villa.

Obs. Les petits plis péristomiens sont souvent peu prononcés; la coquille est quelquefois assez grêle ou très allongée (environs de Magadino, Canobbio, etc.). A Locarne et à Bellinzone (Suisse italienne) il n'est pas rare de trouver la mutation à test paraissant recouvert d'une poussière blanchâtre (Claus. pruinosa, Parreyss). J'en ai récu aussi de Zürich envoyée par M. le prof. Mousson.

(a) Coquille mince, assez solide, peu transparente, brunåtre, á rides longltudinales saillantes, fines et rapprochées; péristome tout plissé.

Lunelle peu arquée; plis palataux 2 ; lamelle spirale séparée. Clausillum sans éehanerures, ni lobes. Mâchoire faiblement striée; bord inférieur obtusement rostriforme. 
Sect. I P II G E I A. (a)

(Iphigenia, Gray - Nat. arrang. Molluse. in Med. repos., XV, 1824.;

a) Andrrea, Henry et Arthur Adams (The genera, etc. loco citatn, 11: 1855, pag. 181).

\section{* C. Clausilia dubia.}

Clausilia dubia, Draparnaud - Hist. Moll.; 1805 , pag. 70.

a) olosoleta. Ad. Schmidt - Die kritischen Gruppen der Europ. Clausilien; 1, 1857; pag. 40 , pl. V, fig. 90 et 91 - Lamella infera, plica palatali, infera, callo palatali evanescentibus (typica).

V. C. - Vallée de la Toce: Montagnes du Verbano, ou Lac Majeur, 280-1000m (Strobel) - Val du Cervo: Andorno, au dessus de Biella, $600^{\mathrm{m}}$ (Cesali).

Obs. D'après M.' Rossmässler (b), le veritable type de la Clausilia dubia, Draparnaud, serait la mutation obsoleta de Ad. Schmidt; et la Claus. rugosa, C. Pfeiffer (non Drap.) en serait la mutation plus robuste, et à lamelle inférieurc bifurquée en arrière et en avant (assez fréquente en Carniole, Tyrol, elc.). M. ${ }^{r}$ Schmidt, au contraire, cite la Claus, rugosa, C. Pfeiffer, comme identique de Clausilia dubia, Drap.! Je crois que M. ${ }^{\mathrm{r}}$ Rossmässler ait raison! La mutation obsoletu est la forme qu'on rencontre plus rommunement dans toutes nos Alpes.

(a) Coquille le plus souvent ridée, à sutures non papilliferes; de couleur bran-corne ou brun-noirâtre. Lunelle développée, arquée. Plis palataux 4 à 2. (le plus souvent un seul); lamelle spirale courant dans la même direction de la lamelle supérieure, unie avec elle. Clausilium non lobé ni échancré. Màchoire à stries vertleales peu prononcées, et à hord inférieur obtusement rostriforme.

(b) Rossmässler : Jconogr. etc., VIII, 1838 ; pag. 23 , pl. 34 , fg. 479. 


\section{ซ. Clausilia nigrieans.}

Turbo nigricans, Pulteney - Cat. Brids, Shells, etc. Dorsetshire, elc.; 1799, p. 46. Claus. obtusa, C. Pfeiffer - Naturgeschichte deutscher Land und Sïssw. Moll. ; I, 1821. Cl. nigricans, Jeffreys - Syn. Test, in Transact. Linn. Soc. 1828; Ad. Schmidt Die krit. Grupp. Europ. Claus., loco citato, I, 1857; pag. 47, pl. VI, fig. $110-114$.

V. C. - Val Stura de Lanzo: Ceres, $700^{\mathrm{m}}$; contre le vieux murs, sous la mousse dans les endroits frais et ombragés; avec la Claus. Verbanensis, etc.; assez rare (a). Je l'ai trouvée aussi dans les alpes au dessus de Balme, $1700^{\mathrm{m}}$; sous les grosses pierres (crystallines) associée à la Claus. plicatula, alpina, etc. Rare.

Obs. Les quelques individus que j'ai trouvés dans le dites localités, sont identiques aux exemplaires de l'Annover que j'ai récus de la bonté de M. ${ }^{\mathrm{r}}$ Adolphe Schmidt.

b) Plleaphora, H. et A. Adams - (loco citato, pag. 183).

\section{Clausilia Mellae.}

Testa umbilicato-rimata, ventrosulo-fusiformis, solidula, crebro striata, superne striis validioribus exarata; anfractibus ultimo et penultimo, sub lente, eleganter malleatis; nitidula, cerasina, raro-strigillata. Spira sursum breviter attenuata. Anfractus 9-10 convexiusculi; sutura distincta; ultimus antice costulato-striatus et, pone suturam, tumidiusculus; basi gibbus, medio impressus. Apertura ampla, subcircularis, fuscula; sinulus parvulus, subcompressus. Lamella supera mediocris, cum spirali conjuncta; infera crassiuscula, fuscula, antice sæpe sub-dichotoma ascendens; spatium interlamellare non, vel obsoletissime 2-3 pliculatum; plica palatalis 1 supera, ultra lunellam producta; subcolumellaris flexuosa, emersa; callus palatalis, supra

(a) Lamella infera antice crassiuscula, obsolete bifurcata; spatium interlamellare lave, vel uni-pliculatum. 
validior, margini parallelus, haud raro plicam basalem plus minusve imperfectan, lunellam versus ascendentem, emittens. Lunella arcuata. Peristoma continuum, appressum.

Coquille fusiforme, ventrue, raccourcie et un peu brusquement attenuée au sommet, mince, médiocrement solide, pourvue d'une fente ombilicale courte. Test assez transparent, un peu luisant, d'un brun-rougeåtre, à rides longitudinales rapprochées, peu égales, légèrement flexueuses, fines, plus fortes sur la moilié supéricure de la coquille; les dernier et avant-dernier tours paraissant comme trèillissés; quelques linéoles courtes, rares, blanchâtres partent des sutures. Spire composée de 9-10 tours, un peu convexes, à sutures assez marquées; le dernier tour à rides plus fortes sur le devant, ct présentant près de la sulure une légère élevation linéaire; médiocrement sub-bigibbeux à la base, et avec une compression au milieu; vers la région ombilicale la gibbosité est plus saillante. Ouverture large, presque circulaire; goutlière petite, arrondie-ovale, subcomprimée. Lamelle supéricure médiocre, atteignant la marge du péristome, courant dans la même direction de la lamelle spirale et sondẻe avec elle; lamelle inférieure assez épaisse, médiocrement callcuse au bout, souvent bifurquée en dehors (branches peu apparentes). Plis interlamellaires nuls, ou bien 2 à 3 peu marqués. Un seul pli palatal qui se prolonge au delà de la lunelle; pli subcolumellaire flexueux, emergé; callosité palatale brun-roussàtre, parallèle à la marge de l'ouverture, plus robuste sur le devant du dernier tour; dans plusieurs individus celte callosité s'extende à la base en forme d'un pli plus ou moin rudimentaire (comme dans la Claus. lineolata, Ileld), ou plus ou moins développé (comme dans la Claus. densestria. $t a, Z_{\text {. }}$ qui se dirige vers la lunelle. Celle-ci est arquée. Péristome continu, rebordé, comprimé, blanc-roussâtre.

Hauteur: 10 mill.; Diametre: 3 mill.

Ouverture: Haut. 2; Diam. vix 2 mill.

Clansilium. Pédicule, long de $1 \frac{1}{2^{m m}}$, grèle, arqué. Lame, long. $1 \% / 2 \mathrm{~mm}$, oblongue, un peu arquée, asse\% solide, non échancrée ni bilobée; légèrement attenuée vers l'insertion du pédicule, ovale-arrondie à l'extremilé, oblusement anguleuse du côlé externe. 
R. P. - Haute vallée du Po: au-dessus du village de Crisolo, pàturages alpestres au pied du Monte Viso, près les ruisseaux, sous les pierres (crystallines), à $1700^{\mathrm{m}}$ (Stabile).

V. T. - Val Maira: au-dessus de Dronéro, $600-680^{\mathrm{m}}$; parmi les mousses (Mella).

Obs. Cette jolie Clausilie (que nous délions avec plaisir à notre honorable ami le comte Charles Vella de Vercelli, qui nous a communiqué plusieurs renseignements malacologiques sur le Piémont) ne peut être comparée à aucune autre espèce de la section Iphigenia, group des Plicaphores (c'est à dire à celles qui ont la base du dernier tour et l'ouverture arrondies). Pour la forme de la fente ombilicale et de l'ouverture, et même pour le pli basal, lorsqu'il est bien marqué, notre espèce offre quelques ressemblances avec la Clausilia densestricuta, Ziegler, de la Carniole; la Claus. lineolata, Held, presente elle aussi un pli tout à fait rudimental, plus ou moins développé, qui de la callosité interne et basale de l'ouverture descende un quelque petit peu vers la lunelle; mais notre espèce ne peut nullement être rapprochée ni de la Claus. densestriata, ni de la lineolata, ni de la plicatula! Les rides fines et addossées distingueront, sans autres caractères, la Claus. Mellce de la Claus. lineolata et de la plicatula, qui ont les côtes élevées et écartées. La petitesse de la taille, les stries inégales, la couleur du test, etc. séparent neltement la Claus. Melle de la Claus. densestriata, dont la taille est bien plus grande, les rides sont plus prononcées et régulières, le test non luisant, et de couleur corné-brunâtre un peu terne.

\section{* D. Clausilia lineolata.}

Clausilia lineolala, Held - Beitrag. zur Gesch, d. Weicht. in : Isis; 1836, pag. 275. - Ad. Schmidt: Krit. Grupp. d. Europ. Clausil. etc., 1857, pag. 16.

a) Testa breviuscula, fusiformis, remotius costulata, infra medium costis validis obtu-; siusculis; plica palatali infera, seu basali, obsoletissima, vel nullu apertura subrotundata (= Claus. cruda, quorund., non Ziegler. - Ad. Schmidt: Krit. Grupp. etc., pl. 2, fig. 21).

B) Testa ut in procedenti, sed ventricoso-fusiformis; callo palatali, et plica basali mediocri, crassiusculis; peristomate subinerassato, albo, interdum poreellaneo. 
7) Testa elongatufissiformis, remolius costulata, costis validis, acutiusculis: plica na. latali infera, sen basali, parum conspicua; apertura magis oblonga of minus laba.

V. C. - Valle Anzasca: Calasca, $600^{\mathrm{m}}$; sous les pierres (crystallines) avec l'Hel. nautiliformis; rare. - Val de la Toce: rives du lac Majeur, $200^{\mathrm{m}}$ (Balsamo, Stabile - Mutut. $\left.\beta\right)-$ V. de la Doire Bàltea: collines de Viverone (N-E de Ivrea), $\mathbf{5 3} 0^{\mathrm{m}}$; terrain erratique (Mella) - V. Stura de Lanzo: Viù, $770^{\mathrm{m}}$. Mutat. \& (Bellardi).

R. P. - Environs de Vercelli, $180^{\mathrm{m}}$. Mutat. $\gamma$ (Mella).

Obs. Il parait que la Claus. lineolata, Held, type, (Ad. Sclımidl: op. citat., pl. I, fig. 15-18) n'existe pas au sud des Alpes!

\section{Clausilia plicatula.}

Pupa pliculula, Draparnaud - Tabl. des Moll.; 1801.

Clausilia plicalula, Drap. - Hist. Moll.: 1805, pag. 72; Ad. Schmidt - Kitt. Grupp. Europ. Claus. etc. 18.57, pag. 25, p. III; Moq.-Tandon - op. cis. 1855, pag. 341 (Animal).

a) Testa valide costulata, costis oblusiusculis, mediocriler distantibus ; lamella infera antice ramosa seu dichotoma; plicis inlerlamellaribus 2-3 conspicuis (Ad. Schmidt - Krit. Grupp., etc. pl. III, lig. 43-46 typica).

B) Testa costulata, costis distantibus, validis, obtusis; vel approximatis atque acutiu. sculis; lamella infera antice obsolete bifurcala, vel simplex el incrassala; plicis interlamellaribus $2-3$ medincribus. (Ad. Schmidt - op. cit. pl. III, fig. 47-48).

y) Testa majuscula, lete rufescenti-brunnea, costis validis, plus minusve argutiusculix, intervallis eleganter minute malleatis; lamella infera antice obsolete ramosa seu dichotoma; plicis interlamellaribus \$, vel nullis (Ad. Schmidt op. cil., pl. 111 , flg. 48 !.

o) Testa costis mediocribus, sub-approximatis, arguliusculis; Inmella infera simplex, antice non progrediens; plicis interlamellaribus omnino nullis (Claus. dwbia?, Hartmann, - in Neue Alpina, 1, 1821; non Drap.; teste Pleiffer: Monogr. Helic. viv. Vol. IV, 1859. - Claus. cruda 9, Ziegler; non aliorum; in Ad. Schmidt - op. cit., pl. III. กg. 50. Dans cette flgure la lamelle inférieure n'est pas simple, mais rameuse, et les còtes sont assez distantes entr'elles!).

V. C. - Vallée de la Doire Bàltea: Courmayeur, $1218^{\mathrm{m}}$; Mutatio $\propto$ (Bellardi) - Val de Lys, ou Gressoney: Fontainemore, $780^{\text {m }}$ : 
sur les rochers humides; Mut. $\beta$ (Stabile) - Val de la Stura de Lanzo: Céres, $717^{\mathrm{m}}$; sur les vieux troncs d'arbres, sur les tuiles, etc., etc. Mut. $\gamma$ (Stabile); Chialamberto, $740^{\mathrm{m}}$ (Stabile); audessus du village de Balme, à l'alpe de la Mussa, etc. $1700^{\mathrm{m}}$, sous les grosses pierres (crystallines) avec la Pupa triplicata, la Vitr. pellucida, la Claus. alpina, le Bul. quadridens, etc. - Mut. o (Stabile).

\section{Gen. BALIA. $(a)$}

Balea, Prideaux - in Gray: Zool. journ, 'T. 1, 1824, pag. 61.

Balieu, Leach - Synops. of the Moll. of Great. Brit. 1820 (manoscritto publicato sultanto nel 1852 per cura di Gray).

Bulia, Bourguignat - Aménilés malacol., in: Revue et Magasin de Zoul, par Guérin: Décembre, $1857(b)$.

\section{* 1. Haliu perversa.}

Tubo perversus, Linné - System. Natur.; 1758, Edit. X; 1, pag. 767 ; non Hel. perversa, Linné - ibid. jag. 772 ; nec $H$. perversa, Müller - Verm. Hist. 1774.

Pupa fragilis, Draparnaud - Tabl. Moll. 1801; et Hist. Moll. 1805, pl. IV, flg. 4. Balea fragilis, Prideaux - in Gray : op. cit. 1824 .

Balcea fragilis, Leach - Brit. Moll. 1831, pag. 116. (ex Turton)

Claus. perversa, Charpentier - Cat. Moll. Suiss., 1837; non Claus. perversa, C. Pfeiffer; nec Fitzinger.

Balia perversa, Bourguignat - op. cit., pag. 550, pl. 17, fig. 1.

a) normalis

\section{ק) Clom gata}

V. C. - Vallée de la Toce: Orta, $370^{\mathrm{m}}$ (Nlarani); Domo d'Ossola, $310^{\mathrm{m}}$ (Prada); Vogogna, 240 (Stabile; Mut. $\beta$ ); alentours du Lac

(a) Coquille sénestre, fusiforme-conoide, fragile, ayant l'apparence d'une Clausílie, mais sans clausilium et sans plis. Nâchoire faiblement arquée, obtuse aux bouts, \& stries presque nulles, sans denticules à la marge inférieure, mais un peu rostriforme.

(b) Bàis; - mouchete - Testa strig1s canescentibus, ad suturas presertim, ornata. 
Majeur, 200-500m; sur les vieux murs, sur les trones des vieux arbres, après les pluies, avec la Claus. albopustulala, la Pupa dilucida, etc. (Stabile) - Vallée de la Doire Bàltea: collines de Viverone, dans la province d'Ivréa, $330^{\text {m }}$ (Mella) - V. de la Slura de Lanzo; Chialamberto, Ceres, Ala (assez abbondante), 717-1080 ${ }^{\mathrm{m}}$ (Stabile). V. de la Doire Ripaire: Suse, B60 ${ }^{m}$ (Stabile, Mortillet).

V. T. Versant piémontais de l'Apennin (Marani).

\section{Gen. PUP..}

(Pupa, Lamarck - System. des aninı. sans vertíbies; 1801, pag. 88;

Sect. T O IR Q I L I. A $(a)$

(Torquilla, Studer - System. Verzcichn.; 1820, pag. 89.)

\section{Pupa quinguedentata.}

Turbo quinquedentalus, Burn - Mus. Vindobon. test.; 1778, pag. 370.

Bulimus similis, Bruguiere - Encyclop., Vers.; II, 1792 , pag. 355.

Pupa cinerea, Draparnaud - Tabl. Moll., 1801; el Hist. Moll. 1805, pl. III, fig. 53. Clausilia cinerea, Risso - Hisl. nat. de l'Europe mérid.; 1826, IV, pag. 85.

Pupa quinquedentala, Deshayes, in Lamarck - Anim. sans vertibr. VIII, 1838; non Mühlfeldt. - Voir pour l'Anatumie: Moquin-Tandon - Hist. nat. Moll. de France, 1855; pag. 352 , pl. XXV, กg. 16-19.

V. C. - Vallée de la Doire Riparia: environs de Suse, sur les rochers (crystallins), 600-600 ${ }^{\mathrm{m}}$ (Stabile, Mortillet, Strobel, Mousson). Abbondant.

(a) Coquille ovoïle-allungée, assez solide, à sommet atterué et un peu pointu. Ouver. ture plissée. Tentacules inférieurs très courts, et mème quelquefois réduits à des ma. melons. Nåchuire lisse, ou avec des stries verticales peu apparentes; à bord intérleur un peu rostrifurme. 


\section{* P. Pupa frumentum.}

Pupa frumentum, Draparnand - Tableau des Moll. 1801.

VAR. meridionalis, Strobel - Notizie malacostat. del Trentino; 1851, p. 37.

a) tritleum, Ziegler (P. frumentum, var. illyrica, Rossmässler - Iconogr. d. L und. Sussiv. Moll. Heft. V, 1837, fig. $311 \mathrm{et} 312$ - Adolphe Schmidt: Geschlechtsapparat der Stylomm. 1855, pag. 42, pl. X, fig. 81. (Anatom.)

R. P. Plaine du Po (nord): Mezzana-Corti, près la Cava, (Strobel; (sud): Stradella (Strobel).

V. T. - Val del Tànaro (Strobel) - V. de la Bòrnida: Acqui (Villa); V. Staffòra; Val Coppa; Val Aversa; Val Tidone (Strobel).

\section{Pupa apennina.}

Pupa apennina, Charpentier - in Küster: Chemnitz Conchyl. cabinet, etc. Pupa ; pag. 105 , pl. 14, fig. 22-58; L. Pfeiffer - Monogr. Helic. vivent.; 1853, Vol. III, pag. 545 .

V. T. - Vallée de la Scrivia: Tortone et Corniasca, sur les coteaux de la rive droite (Nortillet). Exemplaires un peu ventrus et raccourcis, identiques à ceux de Ascoli que j'ai récus de l'obligeance de M.' le prof. Orsini.

\section{* Aupa avenacea.}

Bulimus avenaceus, Bruguière - Encyclop. Method., Vers; V1, 2. ${ }^{e}$ partie, 1792.

Pupa avena, Draparnaud - Tabl. Moll., 1801; Hist. Moll. 1805, pl. III, fig. $47-48$. Pour I'Anatomie, etc. voir: Moquill-Tandon - Hist. Moll. France, etc. 1855, pag. 357-358; pl. XXV, ng. 33 ; et pl. XXVI, flg. $1-4$.

a) normalis - (Haut. 6 millim.)

B) major, cornea - (Haut. 6.8 millim.)

V. C. - V. de la Doire Bàltea: Gressoney, 1400-1670m sur les rochers gneissiqques (Stabile). Les (12) individus que j'ai récolté dans 
cctle localité ont l'ouverture plus arrondie, c'est à dire non anguleuse, à la base; et un quelque exemplaire seulement presente, outre les trois plis palataux, un quatrième pli rudimental supérieur.

Vallée de la Doire Riparia: Suse, $500-600^{\mathrm{m}}$ (Mortillet, Strobel); au dessus du village de Rochemolles (Mont-Thabor), $1660^{\mathrm{m}}$; sur les rochers (crystallins), avec l'II. rupestris (Stabile); au Pas-des Echèlles, $1700^{\mathrm{m}}$ (Strobel).

V. T. - Val Maira, $1000^{\mathrm{m}}$ (Mella) - Val Tidone: Monte Pénice, Mut. $\beta$ (Strobel).

\section{Pupa secale.}

Pupa secule, Draparnaud - Tabl. Moll., 1801; el Hist. Moll., 1805, pl. III, fig. 49-50; Rossmässler - Icon. d. Land. und. Sussw. Moll., Heft. V, pl. 23 ng. 317. Moquin-Tandon. - op. cit., pag. 367, pl. XXVI, fig. 36-29. (Animal).

a) minor (Haut: 6 millim; Diam. $2 \%$, tenuiuscula, fere glabra; plicis palatalibus 3 conspicuis, quarta (superaj bretissima, remola.

V. C. - Vallée de la Stura de Lanzo: région alpine $\left(1700^{\mathrm{m}}\right)$; sous les grosses pierres crystallines près les ruisseaux, avec les $\mathrm{Cl}$. plicala et alpina, le Bul. 4 dens, etc. Rare.

Obs. Les quelques individus que j’ai trouvés ici, diffèrent de la Pupa secale du versant nord des Alpes par leur taille plus petite, et le test presque lisse; tandis que les exemplaires nordiques (Béx, Zürich, en Suisse; Rheden en Hannover, etc.) sont tous fortement striés, à stries addossées et obliques. Dans ces localités septentrionales cependant la Pupa secale présente rarement le petit quatrième pli supérieur; au contraire dans les individus de la vallée (méridionale) de Lan $s$, il n'y manque presque jamais.

\section{Pupa viortilleti.}

Testa oblique rimata, sub-cylindracco-fusiformis, glabriuscula, nitidula, cornea. Spira sensim attenuala, acutiuscula; anfractus 9 vix convexiusculi, suturá mediocri distincti; ultimus vix angustior, pone 
rimam compressus. Apertura semiovalis, 7 plicala; plica angulari (ad insertionem anguli externi peristomatis) mediocri, vel brevissima et crassiuscula ; 1 in medio parietis aperturæ, immersa, longe intrante; columellaribus 2 approximatis, remotis, dentiformibus; palatalibus sub-3, supera (sufuram versus) profunde immersa, brevissima, valida, interdum in appendicem longam et tenuiusculam profunde intrante, antice vero ad marginem peristomatis callum emittente, cum hoc tamen non conjuncta; plica palatali altera (seu media) longa, valida, ad marginem usque aperluræ progressa; terlia (nempe infera, seu basali) punctiformi. Peristoma tenue, vix crassiusculum, reflexum, breviter expansum, albido-labialulum.

Coquille fusiforme, un peu cylindracée, régulièrement attenuée au sommet, à fente ombilicale étroite, oblique, assez longue; très peu ventrue, et peu épaisse, presque lisse, médiocrement luisante, assez transparente, d'un corné plus ou moins clair, souvent avec une teinte blanchâtre sur le devant du dernier tour. Spire composée de 9 tours, à peine un peu convexes, le dernier proportionnellement moins grand que l'avant-dernier, comprimé et obtusement caréné à la base. Ouverture demi-ovale, un peu étroite, munie, d'un pli supérieur, plus ou moins long et épais, à l'angle du bord extérieur du péristome; un pli assez long, immergé, sur le milieu du parois ventral; deux plis columellaires raprochés, immergés; trois plis palataux, dont le supérieur (vers la sulure) très immergé, très court el calleux, souvent continué, plus mince et très long, en arrière; vers le bord du péristome souvent une callosité peu apparente en parait la continuation en dehors, mais il y a toujours une interrution; le second pli palatal (celui du milieu) est long et fort et arrive jusqu'au péristome; le troisième ou basal, près la compression cervicale, est très petit el rudimentaire. Péristome interrompu, médiocrement réfléchi et évasé, non épais, sans bourrelet, blanchàtre, à extrémités un peu convergentes.

Haut. : 6, a 7 millimitres - Diam.: 2 , à $21 / 2$, millim.

V. C. - V. de la Doire Riparia: Suse, $300-600^{\mathrm{m}}$; contre les rochers calcareo-talqueux, avec touffes d'herbe, mêlée à la $P$. avenacea (Mortillet, Strobel). 
R. P. - Paturàges alpestres au-dessus de Crisolo, au Monte-Viso, a $1600-1800^{\mathrm{m}}$; sous les grosses pierres (crystallines) près les ruisseaux et les touffes herbeux (Stabile).

V. T. - Val Stura de Cuneo, ou Démonte: Vignolo, Bsou; et Rocca-Sparvéra, $560^{\mathrm{m}}$ près de Cuneo (Ilortillet) - Val du Gesso (V. Vermegnana): Vernante, au pied du Col de Tenda (Strobel).

Obs. La P. Mortilleti est bien voisine de lat P. multidentata, Olivi (variabilis, Drap.), dont n’est, peut-être, qu'une interessante varieté locale. Cependant on distinguera aiscment notre Pupa Mortilleti à sa taille toujours grèle et petite, non renflée, mais fusiforme comme le $P$. avenacea, et mème un peu subeylindrique; à son test peu sulide, à son péristome non épais, à ses plis palataux en nombre de 3 (et non 4 comme dans la $P$. multidentata); sa forme d'ailleurs est conslante, et il n'y a pas de individus qui faissent le passage entre la $P$. Mortilieti et la multidentata; enfin celle dernière n'a pas encore été trouvée en Piémont, tandis qu'elle est commune au delà des Alpes et des $\Lambda$ pennins. Lne variété de la $P$. multidentata que M. ${ }^{r}$ Bourguignat désigna sous l'appellation de Sabaudina (a), se distingue: par son test plus petit, par son ouverture plus large, par ses quatre plis palataux excessivement immergés, à l'exception du troisième qui, scul, sous la forme d'un forte lamelle, vient s'epanouir sur le péristome. Hème ectle variélé ne peut nullement être confondue avec notre espèce. La $P$. variabilis de Suse et de Vernante citéc par M. ${ }^{\mathrm{r}}$ Strobel est, sans doute, notre $P$. Mortilleti.

\section{* P. Pupar granum.}

P'tpa granum, Draparnaud - Tabl. des Moll. 1801; et Hist. Moll. 1805, pl. II, fig. 45, 46. - Rossmïssler: Icon. d. Land. und. Süssw. Mollusk., Ileft. V, 1837, pl. 23, fig. 322 - Moq-Tandon - Op. citat. 1855, pag. 370 Inimal, pl. Xidve, flg. 3ł-38.

Obs. M. Strobel cite celte espèce trouvéce par lui dans la "Valle del Ticino" transportée par le Pò et le Ticino. Je ne doule pas que

(a) Bourguightt - Malacologie d'Aix-Les-Bains; Paris, 1864, pag. 48, pl. 2. fig. 6-7. 
eetre pelite espẻce doit se trouver dans quelques-unes des vallées leanspadanes sur le versant nord de l'Apennin.

\section{Sect. S IN IX}

(Sphyrudium (partim), Agassiz - in Charpentier: Mol!. Suiss. 1837.)

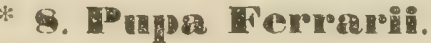

P'upa Fevrari, l'urro - Malacologia Comasca, 1538; pag. 57, tav. 1, lig. 4.

Sphyradium Ferrari, Hartmann - Erd. und. Sïssw. Gasterop. I, 1840; pag. 53, pl. 2, fig. 1-2 (peu caractir.).

a) moneralis, mihl - Prospetto Sistem. Stut. Hull. Lugano, 1839 ; Porro loco supva citato, fig. 4 , a. h. c.

3) guttana, Porro - loco cit., fig. 4, d. e.

V. C. - Vallée de la Toce: Arona, còtes occidentales inférieures du Lac Majeur, 210-500 (Prada, Stabile) - Val Sésia (Val du Cervo): Biella, 480 $0^{\mathrm{m}}$ (Cesati) - V. Stura de Lanzo: près le village de Céres, $717^{\mathrm{m}}$; sous Ies pierres micaschisteuses, avec la Pupa pagodula (Stabile).

V. T. - Val Maira: Dronéro, $600^{\mathrm{m}}$; parmi les mousses (Cesati) - Val du Tanaro (Strobel).

Obs. Quoique la localité de Dronéro appartient plutòt à la plaine que aux vallées, cependant la Pupa Ferrarii, la Claus. Melle, ete. doit-on les considerer comme espèces propres au basses régions des montagnes et des vallées, descendant jusqu'à la limite inférieure sans la dépasser.

(a) Coquille souvent pelite, cylindroide, a sommet oblus. Onverture plissie. Tentacules inlerieurs punctifurmes. Mảchoire arque, it peine un neu strice, non rostrée au milicu. 
Sect. A G I I I, mihi. $\langle a\rangle$

Odostomia (partim), Fleming - in: Édinburgh. Encyct. 1814: Pupilli (part.), Lejeh - Brit. Moll. pag. 126, ex Turton 1831.

\section{Pupa pagodula.}

Pupa pagodula, Des Noulins - in: Act. Suc. Limn. de Burdeaux, 1V, 1830, pag. 1 ss: fig. - Rossmässler: Icon. d. Land. und Sïssu. Moll., Heft. V, 1837. pl. 23 , fig. 323 .

V. C. - V. de la Stura de Lanzo: Ceres, $717^{\mathrm{m}}$; avec la $P$. Fer. rarii (Stabile).

Sect. IP I I L I. $\mathbf{A}(b)$

(Pupilla (partin), leach - loco cil.)

10. Pupar musceorum.

Turbo muscorum, Linné - System. Natur., Bilit. X, 1758; 1, pag. 767.

Pıpa marginata, Draparnaud - Tabl. des Moll. 1801; (t Hist. Moll. 1805; pl. III. fig. 36-38 - Russmässler: Icon. Land. und Süssw. Moll., Heft. V, 1837; pl. 23, fig. 323 - Moquin Tandon: Hist. Moll. de France, 1855, p. 393. Anatomie).

V. C. - V. de la Toce: Domo d'Ossola, 306 (Prada) - V. de la Doire Ripaire: Mont-Cénis (Strobel).

R. P. - Plaine du Pó (nord): environs de Turin (alluvions et aval), $200^{\mathrm{m}}$ (Mortillet) - Plaine sud du Pô: Alexandric, 86-90 (Strobel).

(a) Coquille petite, cylindrique-ovoide, mediocrement ventrue; ombilic itroit. long; ouverture sans plis; dernier tour de spire plus grand que l'avant-derniel. muntant vers le sommet de la coquille. Pednincules, ou tentacules supirieurs, trés-longs. inférieurs assez développes. Piod muni de muscles assez. forts. Jìchoire faithement arquée, à stries presque invisibles.

(b) Coquille petite, subcylindrig̣ue, un peu ventrue. Ouverture avec quelques pelites dents. Mà hoire presquue lisse, à bord inféricur lablement rostri, cu sins rostre. 
V. T. - Val Tidone: Zavatarello (Strobel, Prada).

VAR. B. - alpieola, Charpentier - Mollusq. terr. et fluv. de la Suisse; 1837, pag. 16 , pl. 2, fig. 5 - Lin petit peu plus petite que la vraie $P$. muscorum, Linn., et avec le péristome à bourrelet moins robuste et moins jaunâtre.

a) indentata - Pas de dents ou de callosité.

B) unadentata - Une dent ou callosité vers le milieu de l'avant-dernier cour a l'entrée de l'ouverlure.

$\gamma$ ) bidentata - Uno dent à l'entrée de l'ouverture, vers le milieu de l'avant. dernier tour; et une dent palatale inférieure opposée à l'autre.

V. C. - Au-dessus de Balme dans l'haute vallée de la Stura de Lanzo, $1500-1700^{\mathrm{m}}$; sous les grosses pierres(crystallines), avec l'espèce suivante. Pas commune (Stabile). Il faut probablement rapporter à la Var. alpicola, la Pupa muscorum trouvée par M. ${ }^{\mathrm{r}}$ Strobel au Mont-Cénis.

Obs. La $P$. muscorum Linn., typique, présente elle aussi les trois mutations nommées ci-dessus. La mut. bidentata est un peu rare en Lombardie et en Suisse ( $P$. bidentata, C. Pfeiffer - Systemat. Anordn. und Beschreib. deutsch. Land-und Wass. Schnecken 1821). La mut. unidentata, Pfeiffer C., est la plus commune et la plus répandue. Quelques auteurs (Ad. Schmidt, Moquin-Tandon, etc.) donnent à la mut. bidentata le nom aussi de $P$. bigranata, Rossmässler (Iconogr. Heft. IX et X, fig. 64B). Plusieurs autres, au contraire, (L. Pfeiffer, Strobel, Gredler, etc.) donnent le nom de bigranata à la mutation de la $P$. triplicata, Studer, pourvue seulement de deux plis, en lieu de trois, à l'ouverture.

\section{Pupa triplieata.}

Pıpa triplicala, Studer - Kurzes Verzeichn. der Schweiz. Conch, 1820.

- Iridentalis, Michaud - Complém. à Drap. 1831, pl. XV, fig. $28 \cdot 30$; Rossmässler: Iconogr. Heft. V, 1837, pl. 23, fig. 324; Moquin-Tandon: Moll. de France, 1855, pag. 395 (Animal).

V. C. - Balme, dans la vallée de la Stura de Lanzo, 1500-1700 avec la $P$. alpicola, Chp. (Stabile) - Très rare, mais parfaitement typique.

V. T. - Vallée du Tanaro (Strobel). 


\section{* 12. Prpar Sempronii.}

Pupa Sempronii, Charpentier - Cat. Mull. Suiss.; 1837, pag. 15. 11. 2. Nig. 4. (6s).

a) normalis.

B) dilucida, Ziegler (in Rossm.: Icon. Heft. V, 1837, ng. 326)-Peristumale repexiusculo, tenui; dente rudimentali vel nullo.

V. C. - Versant méridional du Simplon: Gondo, 700-800 les rochers granitiques; mutation $\propto$ (Venetz, Charpentier) - Rives occidentales du Verbano, 220-500 $\mathrm{m}$; sur les vieux murs, sous la mousse, etc., avec la Balia fragilis, pendant la pluie; mut. \& (Stabile) - Valléc de la Doire Bàltea: Róppolo, collines de Viverone, $530^{\mathrm{m}}$; sur les vieux murs à sec (Mella) - Environs de Turin, alluvion, aval, $200^{\mathrm{m}}$ (Mortillel). Partout elle vit sur les pierres crystallines.

Obs. Celte espèce ne différe de la P. umbilicala, Drap., que par sa taille moins robuste et plus petite, par son péristome moins blane et peu épais; relle forme, cependant, est constante. Quant à la présence ou absence plus ou moins absolue du petit pli, ou dent, de l'avant-dernier tour, touchant l'extrémité du bord extéricur du péristome, j'ai déjà fait observer, lorsque j'ai parlé de la Crecilianella acicula, que celte pièce accessoire a peu d'importance pour la déterminalion d'une espèce, parceque clle varie beaucoup selon l'âge du mollusque, les conditions physiques où il vit, etc. La chose va bien autrement pour les plis de certaines autres espèces, du nombre et de la forme des quels on doit tirer les caracteres pour la déterminations des espèces mêmes.

(a) Testa cornea, nitida, cylindrica, obtusa; apertura unidentata: peristomate albido. fucescenti, repexo, plano. Alt. 3 a $3 \frac{1}{9}$ : Diam. $1 \frac{3 / 8}{4}$ millim. 


\section{Gen. VERTIGO. (a)}

(Terligo, Muller - Term. Hist.; Il, 1774 - Pupa (partim), Drap. - Tabl. Mull. 1801.)

Sect. I S T H I I A . (b)

(Isllmia, Gray - Nalus. arrang. Moll.; in: Medical. Repository; 1821, XV.)

\section{Vertigo erlentula.}

Pupa edentula, Draparnaud - Hist. Moll. de France; 1805, pag. 52, pl. III, fig. 28-29. Vertigo edentula, Studer - Kurzes Verzeich. Conch.; 1820, pag. 89.

- nitida, Férussac - Tabl. System. Moll.; 1822, pag. 68. - Rossmässler : Iconogr. Land-und Süsswass. Moll.; Heft. IX et X, 1839, pl. 49, fig. 646 - No. quin-Tandon: Mollusques de France, 1855, pag. 402 (animal); pl. 28, fig. $28-30$.

V. C. - Vallée de la Toce: Bavéno, $210^{\mathrm{m}}$ (Benson) - V. de la Stura de Lanzo: au-dessus de Balme, $1500^{\mathrm{m}}$; sous le pierres (erystallines) associée à la Pupa tiplicata, Hyalina fulva, etc., assez rare partout (Stabile) - Vallée de la Doire Ripaire: Mont-Cénis, 2000 (Strobel).

R. P. - Plaine du Po (nord): Vercelli, $150 \mathrm{~m}$; terrain erratique (Mella); Turin, $220^{\mathrm{m}}$; alluvions, avàl (Mortillet).

(a) Les Vertigos diffèrent des Maillots, ou Pupes, par l'absence des tentacules inféricurs. II y a des Pupes très pelites, de la taille mème des Vertigos, et cependant elles ont les tentacules inférieurs assez dévoloppés; mais chez les Tertigos ces organes n'existent pas, et ils ne sont pas même représentés par une saillie rudimentale; on n'y voit quelquefois que une tache noirâtre. Mâchoire faiblement arquée, sans striès, ni denticules marginales.

(b) Mâchoire sans apparence de saillie rostriforme au milieu du bord inférieur. Copiulle exlindrique. 
* 2. Vertigo musconum.

Pupa muscorum, Drap. - Tabl. Moll., 1801, non Linne, nec Mulfer, Ne: Lamk. - minutissima, Hartmann - in Neue Alpina; 1821, pag. 220, 11. I1, lig. 5. Yertigo cylindrica, Férussac - Tabl. System., 1822 - Moquin-Tanilun: Moll. de France, 1855, pag. 400 (anim.), pl. 28 , fig. 20.24.

R. P. -. Plaine du Pú (nord): Vereelli (Mella); Val du Ticino (Strobel); Turin, alluvions (Hortillet) - Plaine sud: Alexandrie, Vallée du Tanaro (Strobel).

Sect. IE X IO G Y I A, mihi. $(a)$

\section{Vertigo TIoulinsiana.}

Pupa Moulinsiana, Dupuy - Catul. extramar. Gallice testac; 1849, X. $28 \%$.

- Charpentieri, Shutllworth - in Kister: Conch. Cab., 18:2: pag. 129, pi. XV., fig. $41-43$.

V. T. - Val de la Scrivia: Stazzano près de Serravalle, (à peu près $\left.150^{\mathrm{m}}\right)$ (doct. ${ }^{\mathrm{r}}$ Ferrari de Gènes; Mella).

Obs. La Vert. Moulinsiana se distingue de ses affines par sa taille plus grande, par son ouverture munie de 4 plis ou denticules seulement; 1 dent immergée, vers le milieu de la convexité de l'avantdernier tour; 1 columellaire (il existe rarement un'autre petite denticule vers la base de la columelle); et 2 palatales. Celte jolie espèce cosmopolite (b), comme presque toutes les Verligos d'Europe, n'a pas encore été trouvée dans la région cispadane du Piémont, ni en Lombardie! Est-ce que, existant dans certains endroits seulement, elle a échappée aux recherches des conchylliologistes?

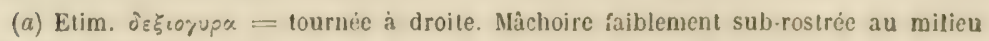
du bord inférieur.

(b) Je l'ai réçue de divers points de la France (nord et midi); de l'Espagne; de la Sicile; de la Suisse uccident (Béx, Berne, etc.). 


\section{* Vertigo pygmaea.}

Pupa pygmaa, Draparuaud - Tabl. Mollusq., 1801; et Hist. Moll., 1805; pl. 11, fig. $30-31$.

Verligo pygmaza, Férussac père - Essai d'une méthode, etc. appliquée aux Moll. terr. et fluv., etc.; 1807, pag. 124. - Rossmässler : Icon. Land-und Süssw. Moll., Heft. IX et X, 1839, pl. 49, fig. 648. - Moquin-Tandon: Hist. Moll. de France, 1855 , pag. 405 (Animal).

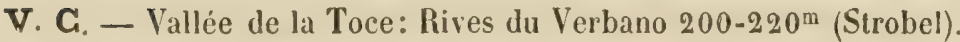

R. P. - Plaine (nord) du Pò: Vallée inférieure du Tessin, $80-120^{\mathrm{m}}$ (Sirobel); Vercelli, $150^{\mathrm{m}}$; terrain erratique (Mella) - Haute vallée du Pó: alpes de Crisolo, pied du Mont-Viso, $1500^{\mathrm{m}}$, sous les pierres cryslallines et sur les débris de bois pourri, près les ruisseaux, dans les endroits bumides et marécageux, etc. (Stabile).

\section{* 5. Vertigo antivertigo.}

Pupa antiverligo, Draparnaud - Tabl. des Moll., 1801, pag. 57.

Vertigo sexdentata, Férussac père - Essai méthod. etc., loc. cit., 1807, pag. 124.

- septem dentata, Férussac - Tabl. Systém. 1822, pag. 68. - Moquin-Tandon: loco citato, pag. 407. (Animal).

V. C. - V. De la Doire Bàltea: Liliannes, en Val Gressoney, $650-670^{\mathrm{m}}$; sous les pierres, sur les débris de bois pourri, sous les feuilles pourries, etc. (roches crystallines), rare partout (Stabile).

R. P. - Basses régions du Tessin: 80-120 ${ }^{\mathrm{m}}$ (Strobel); Vercelli (Mella).

V. T. - Val Varaita: Brossasco près Venasca, \$40-600 (Mort.).

Obs. Même gisement des espèces précédentes. Quelquefois existe un pli rudimentaire au-dessous des plis columellaires, à la base de l'ouverture, et un autre, très petit, dentiforme, palatal supérieur vers l'angle de l'ouverture (Rossmässler: Icon. d. Land-und Sïssw. Moll., Heft. X, 1839; pl. 49, fig. 647). 
Sect. VI I T I L I. $(a)$

(Vertilla, Moguin-Tanton - Ifist. Holl. de France, 1855, Mig. 408.)

\section{Vertigo Venetuit.}

Vertigo Venetzii, Charpentier - in Férussac: Tubleau Systim., 182:-2 par. 69.

- plicata, Auguste Muller - in Wiegmann: Archiv. für Naturyeschichte, 1820, pag. 210, pl. $1 \mathrm{~V}$, ก.g. 6.

- Venetzii, Charp. - Catal. Moll. Suiss. 1837, pag. 18, pl. 2, flg. 11.

- hamata, IIeld - in: Isis, 1837, pag. 304.

Rossmässler - Icon. der Land-und Süssw. Moll., Heft IX el X, p). 49, fig. 63̈.

Moquin-Tandon - Moll. de France, 1855, pag. 408 (Animal).

R. P. - Vallée du Ticino (Strobel).

\section{* Vertigo pusilla.}

Vertigo pusilla, 0. Müller - Term. Hist., 11, 1774, pag. 129.

Helix vertigo, Gmelin - Systema naturce, etc. 1788, pag. 3664.

Pupa vertigo, Draparnaud - Tabl. Moll., 1801, pag. 57.

Rossmässler - Iconogr. etc., loco citato fig. 649 .

Moquin-Tandon - Op. cit., pag. 409 (Animal).

V. C. - Rives du lac Majeur (Strobel), Feriólo (Benson) - Yalléc de la Doire Baltea: Viverone (Mella).

(a) Coquille tournée à gauche, trés petite. Matchoire i horl lihre, ou infirienr, a peine un petit pen rostrifurme-arrondi au milieu. 
†† Oculos ad basin pedunculorum ferentia.

$\dagger$ Oculi ad basin postero-internam.

\author{
FAmilia: A U R I C U L A C E A.
}

Gen. CARYCHIUM.

(Carychium, Mülter -- Verm. Hist. , II, 1774, pag. 125.)

\title{
* Caryelnium minimum.
}

Carychium minimum, Müller - Verm. Hist., loco citato.

Auricula minima, Drap. - Tabl. Moll., 1801, pag. 54.

Rossmässler - Icon. etc., Heft IX et X, 1839; pl. 49, fig. 660

Moquin-Tandon - Moll. de France, 1855, pag. 413, pl. 29, fig. 15.23 (Analom).

V. C. - Val de la Toce: Domo d'Ossola, $510^{\mathrm{m}}$ (Strobel) - Val de la Doire Bàltea: Viverone, $330^{\mathrm{m}}$ (Mella).

R. P. - Plaine du Pô, nord: Valle del Ticino, $60-100^{\mathrm{m}}$ (Strobel) -; Vercelli, $130^{\mathrm{m}}$ (Mella); Turin, alluvions, $220^{\mathrm{m}}$ (Mortillet).

\section{Carychium tridentatum.}

Saraphia tridentata, Risso - Hist. nat. Europ. mérid., IV, 1826 , pag. 84.

Carychium elongalum, Villa - Dispos. syst. Conch., 1841, pag. 59.

- tridentatum, Bourguignat - Aménilés malacologiques, in : Revue et Mag. Zool. par Guérin, 1857, § LXIV; et 1859, §XXII, pl. II, fig. 12 (tirage à part, pl. XV, fig. 12).

V. C. Hôspice du Simplon, $2020^{\mathrm{m}}$; sous les grosses pierres dans les endroits marécageux (Stabile). Six exemplaires qui ne diffèrent pas l'un de l'autre. Celle espèce (ou variélé (?) de la précédente) a été trouvée aussi par NI. ${ }^{r}$ Bourguignat dans les bois de Schwibbogen, entre Enatten et Sonnenberg près du lac de Lucerne, ou lac des Quatre-Cantons, (entre 750 et $8 \mathrm{~B}^{\mathrm{m}}$ ); et à Giessbach, dans l'Ober- 
land Bernois, pris des cascades (à peu près $600^{\mathrm{m}}$ ). Nuus avons reçu ce Carychium de Florence, recueilli el communiqué par notre ami conchyliologiste M. ${ }^{r}$ Czielli de Livourne; sa forme est un pelit peu plus élancée, et ses tours paraissent un peu moins convexes. Le $C$. tridentatum existc aussi en Lombardie, où il est mèlé quelquefois au C. minimum. D'aprés Moquin-Tandon (Moll. de France, p. 414) M. ${ }^{\mathrm{r}}$ Puton aurait trouvé le $C$. minimum dans les Vosges à $800^{\mathrm{m}}$; il est bien probable que ce soit le $C$. tridentatum.

\section{B. PULMONATA OPERCULATA. ${ }^{(7)}$}

t† Oculi ad basin postero-externam.

\section{Familia: A C I C U L A C E A.}

\section{Gen. ACWE.}

Acme, Hartmann - System. Gasterop. 1821, pag. 37-Aicula, IIartmann in: Neue Alpina, 1821: non Risso - Pupula, Agassiz, in Charpentier - Moll. terr. et fluv. de la Suisse, 1837.)

\section{Acme lineata.}

Bulimus lineatus, Draparnaud - Tabl. Moll., 1801.

Auricula lineata, Drap. - Hist. Moll., 1803, l. III, fig. 20-21 (mala).

Acicula lineala, Hartmann - in: Nere Alpina, 1821, I, pag. 215.

Cyclostoma lineatum, Férussile - Dict. class, d'hist. natur., II, 1822, pag. 90.

Acme lineala, IIartmann - in Sturm: Deutschland Faumu, etc. VI, 1823.

Pupula lineata, Charpentier - Op. cit. 1837, jag. 23.

Cyclostoma lineatum, Porro - Malacologia Comasca, 1838, pag. 76, pl. 1. lig. 5.

Moquin-Tandon - Moll. de France, 18.55, pag. 509, fl. 38, fig. 4-7 (optima).

V. C. - Rives occidentales du Lac Majeur: Belgirate, etc.; sous les grosses pierres, dans les petils vallons abrite's et frais; rare (Stabile).

(7) Voyer à la nn du livre. 
t十 Oculi ad basin externam.

\section{- Familia: C Y Clos T O M a C A.}

\section{Gen. Gyclostohus.}

(Cyclostuma (partim), Draparnaud - Tabl. Holl. 1801; Cyclostomes, Montfort - Conch. systém., II, 1810, pag. 287).

\section{Sect. II ICIA}

(Ericia, Moquin Tandun - in Parliot: Mémoire sur les Cyclostomes, 1848).

\section{* Cyelostomus elegans. (8)}

Nerila elegans, Müller - Verm. Hist., 1774, II, pag. 177.

Cyctostoma elegans, Drap. - Tabl. Moll., 1801, pig. 38.

Cyclostomus elegans, Montfort - Conch. syst., 1810, pag. 287.

Moquin-Tandon - Moll. terr. et fluv. de France, 1855, vol. I (études anatom. et physiolog. des moll.), et vol. II, pag. 497, pl. 37, fig. 3.23 (Anatom.).

Troschel, H. - Das Gebiss der Schnecken, etc.; Berlin, I, 1856, pag. 69, pl. 4, fig. 8 (dents linguales).

Claparède, R. E. - Cyclostom. elegantis anatome - Dissert. inaugur., cum tab. 2, Berlin, 1857.

R. P. - Plaine du Pô (nord): Pavie, charrié par le Tessin (Strobel, Rezia) (a); Turin (Mella) - Plaine sud: Rosignano, Sanctuaire de Créa, 200-400 (Mella); collines d'Asti, Alexandrie, Valmadonna, (Mella, Strobel, Marani); Monferrato (Rezia); Stradella, Broni (Villa, Strobel).

V. T. - Vallée du Tanaro (Strobel) - V. de la Bòrmida: Acqui (Villa, Bonomi) - V. Scrivia, sur les coteaux de la rive droite à 'Tortone, Corniasca, etc. (Nortillet) - Val Staffora; Val Coppa; Val Aversa; Val Tidone; Bobbio, etc. in Val Trebbia (Strobel).

(8) Voyez à la fin du livre.

(a) D'après M.' le doct. A. Rezia (Enum. sistem. Gasterop. Lerr. e Muv. dí dintorni di Pavia, 1848) on aurait essayé infructueusement d'acclimater le $C$. elegans à Pavie. 


\section{NOrISs.}

(1) Les mollusquues pulmonés inoperculés (ì l'execption des Proserpinides) sont androgynes, et appartiennent ì l'ordre des Adelopneumones, c'est is dire à ces mollusques qui ont l'orifice respiratoire petit, situé ì droite ou ì gauche de la marye de la cuirasse ou du collier, et uvec un sphineter spécial. (1). Les progrès de la malacozoologie ont fait connaitre anssi les notables différences des organes digestifs, et plus partieulièrement des ingestifs ou de la mastication, dont l'étule est d'autant plus important car ces organes sont en rapport avee les mours et les habitudes de ees petits êtres merveilleux qui sont bien

(1) Chez Ies Inopercules d'eau douse, la cavite respiratuire lapissce d'un riseau vasculaire pour la respiration aírienne, contient aussi quelques lamelles branchiales pour la respiration aquatique; d'apres cette organisation, plusieurs malacolugistes ont rétiré ces mollusques de l'ordre des Pulmonés, el ont créé pour eux l'ordre des Pulmobranches; quant aux Ancyles on les a placés parmi les Monopleurobranches. Si d'un còté il y a des motifs qui justilient ces démembrements, il n'est pas moins vrai, d'autre côté, que les Inoperculés d'eau douce sont de vrais pulmonés, c'est à dire respirants l'air libre.

Mime chez les Operculis terrestres l'appareil vasculaire présente déja quelques rudiments d'organisation branchiale, et nianmoins it sont tous des mollusques démeurants sur les trones des arbres, sur les rochers, etc., mime où il n'y a point d'eau, tandis que les Operculés aquatiques sunt des vrais branchiféres destinés à vivre dans l'eau, et pas autrement. Quand on parle des mollusques, ce H'est pas taxativement et, common dit, a la lettre, qu'un duit accepter le mut pulmonés, cumne l'on ferait pour des animaux d'un ordre superieur; mas, pour tout malacologiste senst, un mollusque pulmone n'est autre chuse gur un mollusque respirant l'air libre. 
dignes de toute l'attention des serutateurs de la nature (1). Nous n'entrerons iei que dans quelques détails deseriptifs sur la mâchoire et la langue des Adelopneumones.

La mâchoire est une pièce arquée, dure, cornée, implantée supérieurement dans les lèvres, c'est à dire fortement attachée aux parois de la cavité buceale. Cet organe est composé de mucus éndurci, contenant une faible quantité de carbonate de chaux. Presque tous les Adelopneumones n'ont que une seule mâchoire, quelques-uns trois; d'autres enfin n'en possèdent pas.

\section{Mâchoire unique ou solitaire.}

Chez les Adelopneumones qui possèdent une seule mâchoire, celle-ci se présente sous des formes différentes, dont voici les principales:

Oxygnatha - Nâchoire fortement arquée, et quelquefois verticalement carénée au milieu, avec une forte saillie rostriforme, plus ou moins large, ou en forme de bec, à la partie moyenne du bord libre ou inférieur.

a) Mâchoire arquée d'avant en arrière, lisse on presque lisse: Limax, Philomycus (2), Tennentia (3), Parmarion, (4), Ilelicarion (5), Vilrina, Zonites.

(1) On a entin compris combien il est indispensable à l'avancement et perfectionnement des études malacologiques, de bien examiner, avant tout, l'organisation des mollusques. Plusieurs savants dans ces dernières années, se sont occupées artivement de celte branche importante de l'histoire naturelle, et leurs travaux ont déjà apporté beaucoup de lumière dans l'obscure labyrinthe; il reste encore bien à faire, c'est vrai, mais nous ne doutons pas d'affirmer que de nos jours on a déjá de quoi poser quelques bases d'une malacologie assez rationelle. Parmi les contemporains illustrateurs de l'anatomie soit générale soit specièlle des mollusques, nous citerons les suivants: Albers, Baudon, Claparẻde, Deshayes, Milne Edwars, Fischer, Gassies, Gegenbaur, Gratiolet, J. E. Gray, Aloys Humbert, Keber, Knight, Lacaze-Duthiers, Laugier, Lebert, Leidy, Lovén, Macionald, Moquin-Tandon, Mürch, Otto Goldfuss, Rubert-Garner, Saint-Simon, Adolphe Schmidt, Oscar Schmidt, Semper, W. Thompson, Troschel, Wilton, Woodward, etc. Malheureusement nous ażons, dans ces dernières années, à regretter la perte de plus d'un de ces honorables savants. Ici à Milan, c'est M.r Sordelli (l'habile dessinateur des reptiles du grand ouvrage de M. ${ }^{r}$ le Prof. ${ }^{r}$ Jan) qui s'occupe actuellement de l'anatomie des mollusques. Ce monsieur a bien eu l'obligeance de se mettre à nutre disposition, pour cette partie, et dès ses premiers essais nous n'avons que des éluges à lui tributer.

(2) Philomycus, Rafinesque - Annal. of nat. 1820 (Limax carolinensis, Bosc.).

(3) Tennentia, Humbert - in Revue et Mag. Zool, par Guérin, Paris; 1862, novembre (T. Twaithesi, H.).

(3) Parmarion, Fischer - in: Act. Soc. Linn. de Bordeaux, tom. XX; juin, 18ü.

(i) Helicarion, (Ilelixarion), Firussae - Tablecu system., Prodrome, 1822. 
b) Máchoire à côtes fines, serrées, n’arrivant pas jusqqu’à la marge infé. rieure: Macrocyclis (1).

c) Mâchoire courbée en forme de fer ì cheval, et munie de côtes fortes et distinctes: Simpulopsis (2); ou de dents robustes et saillantes sur le bord libre: Pellicula (3).

d) Mâchoire comme la précédente, avec, et plus souvent sans deuts, toujours surmontée d'une plaque large et haute, trapezoïde ou quadrangulaire, implantée dans la cavité buccale (4), et tantôt lisse ou presque lisse : Succinea; tantôt avec de côtes verticales: Omalonyx (5).

e) Mâchoire peu courbée, lisse, très semblable à celles des Zonites, mais surmontée d'une plaque quadrangulaire, haute et large, à peu près comme chez les succinćes: Camptonyx (6).

Aulacognatha - Mâchoire peu arquée, souvent assez étroite, à stries fines, plus ou moins prononcées, et à denticules ou crénclures marginales petites, peu distinctes, ou nulles; saillie médiane du bord libre plus ou moins prononcée, non rostriforme.

a) Saillie médiane, denticules marginales et stries nulles: Ariophantı (A. retrorga, Gould; spec. sinistr.).

b) Saillie médiane plus ou moins distincte, quelquefois nulle; strics nombreuses, plus ou moins étendues, apparentes, ou demi-effacées: Parmacellu; IIflix - Sect. Delomplalus (II. rupestris, rotudata, ete.), Bradybana (H. ciliata), Sagda (II. Cookiana, Guscux; II. alligans, Avıus, etc.); Bulimus - Sect. Buliminus (B. obscurus, montanus, ete.), Zebrina (B. detritus, etc.), Chondrula (B. tridens, quadritens, etc.), Azeea (B. Menkeanus, dentiens), Zua (B. subeylindricus), Ferussacia (B. fulliculus, etc.); Stenoryyra - Sect. Rumina (B. decollatus), Subulina (B. octonus, ete.); P'upa, Balia, Clausilia, Carychium, Physa, Planorbis, Amphipeplea, ete.

Odontognatha - Mâchoire à côtes; largement crénclée, ou fortement dentieulée sur le bord libre.

a) Mâchoire médiocrement courbée, à côtes nombreuses, plus ou moins

(1) Macrocyclis, Beck - Index Moll. 1837 (IIelix Laxata, FÉR; II. concava, SAy, clc.)

(2) Simpulopsis (Beck), Shullleworth - Beiträge zur näheren Kenntmiss d. Land-und Süssw. Moll. d. Portorico; in: Millheil. Nalurf. Gesellsch. in Bern; Miirz, 1854. Vitrina sulcosa, Férussac; V. rufovirens, Moricand, cle.).

(3) Pellicula, Fischer - Mélanges do Conchyliologie, in : Act. Soc. Linn. de Bordeaux, janvier 1856 (Succinea depressa, Rang).

(4) Chez quelques Arions et Physes, etc. existe aussi une sorte d'appendice, souvent assez large rilatisement a la partie lihre, implantic dans les levres. La prartie supi. rieure de la mitchuire de la Physa acula est terminte cll forme te triangle.

(5) Omalonyx, Dorbigny - Fischer: loco cilato, 18.56 (Suceinea unguis, W0แB.).

6) Camptony, Benson - in: Annals and Mig. of nat. hist. May, 1838. (C. Theo. bialdi, B.). 
fortes et aplaties, formant autant de denticules ou crénelures marginales emoussées: Arion, Ariolimax (1), Megapelta (2), Vaginulus, Helix - Sect. Trigonostoma (H. angigyra, obvoluta, ete.), Drepanostoma, Fruticicola, partim (H. strigella, cantiana, carthusiana, cinctella, sericea, hispida, etc.), Xerophila, partim (H. apicina, conspurcata, unifasciata, cespitum, etc.).

b) Mâchoire peu arquée, à côtes fines ou lignes convergentes; dents marginales nulles: Liguus (3).

c) MI. arquée, à stries perpendiculaires, nombreuses et robustes : Achalina (A. fulica, etc.).

d) M. à côtes peu nombreuses, fortes: Limicolaria (4).

e) Mr. arquée, à côtes peu nombreuses, saillantes, et à dents (ou saillies) marginales, fortes: Helix - Seet. Ulota (H. fruticum), Xerophila, part. (H. neglecta, ericetorum, pisana, variabilis, ete.), Isognostoma (H. personata), Arianta (H. arbustorum), Campylæa (H. glacialis, frigida, colubrina, cingulata, tigrina, zonata, etc.) (5), Iberus (H. serpentina, ete.), Macularia (H. niciensis, sicana, Codringtoni, etc.), Tachea (H. sylvatica, nemoralis, etc.), Pomatia (H. pomatia, H. cincta, Nüller, etc.); Borus (6).

Goniognatha - Mâchoire arquée, convexe en dessus, plus ou moins concave inférieurement; composée de segments imbriqqués.

a) Segments nombreux, verticaux, en forme de larges côtes, à marge externe convexe, un peu concave à l'interne, convergentes vers le centre: $B u$ limulus (7).

乙) Segments larges, en forme de triangles irréguliers, disposés obliquement, à marge externe crénelée; un grand triangle au milieu de la mâchoire: Orthalicus (8).

\section{Trois mâchoires}

Quelques-uns des Adelopneumones possèdent trois mâchoires, ou plus proprement leur appareil maxillaire est formé de trois pièces; l'une robuste (la

(1) Ariolimax, Mürch - Beiträge zur Molluskenfauna Central-Amerika's; in Malak. Blälter, von Menke et Pfeifrer, 1859, (Limax Columbianus, Govlo.).

(2) Megapelta, Mörch - in: Joum. Conchyl., Paris, 1857, (M. semitecta, Murch).

(3) Liguus, Montfort - Conch. syst., 1810, (Achat. virginea, etc.)

(4) Limicularia, Beck - Index, etc. 1837, (Bulimus Adansoni, Pfeiffer).

(5) M. ${ }^{\mathrm{r}}$ de Saint-Simon (Miscel. Malacol., 2. ${ }^{\mathrm{e}}$ décade, Toulouse, 1856) a bien cu l'obligeance de anatomiser presque toutes ces Campylies; M. ${ }^{r}$ Sordelli de Milan, l'II. frigida et l'H. cincta.

(6) Borus, Albers - Die Heliceen nach natürlich. Verwandlsch. system. geord.; Berlin, 18.50 (Bulimus hæmastomus, Scopous).

(7) Bulimulus, Beck - Index, ete. 1837, Bulimus lita, FÉr.; B. cinnamomeo-linea lus, Moricand, etc.

(8) Orthalicus, Beck - Index, elc. (Bul. gallina sultana, LAMk.). 
vraie mâchoirc), superieure, transversale; les autres dleux, moins fortes, souvent peu développées, inférieures, sont placées l'une ì droite et l'autre ì gauche, et verticalement.

a) Mâchoires lisses; la supéricure le plus souvent avee une saillic rostriforme médiane peu sensible: Limncea.

b) Mùchoires supérieure et latérales papillifères, nou rostrées au milieu: Ancylus.

c) Mîchoires latérales à peine rudimentales: Planorbis corneus.

\section{Mâchoires nulles}

Agnatha - Enfin les mollusques Adelopneumones carnassiers, qui chassent la proie vivante, ne possèdent pas de mâchoires: Testacella, Daudebar. dia, Glandina, Cylindrella.

\section{Langue et dents linguales}

La langue des Mollusques est représentée par une espèce de ruban cartilagineux (1), long, étroit, épais, coneave en dessus, élastique, placé sur le plancher de la eavité buccale, et recouvert d'une membrane mince, résistante, rude, hérisscée d'un grand nombre de petites papilles ehitiueuses (2), solides, plus communément appellées dents. Chez les Adelopneumones ces dents sont à peu près toutes égales, disposées en séries nombreuses transversales, formant une sorte de pavé uniforme ou trèillisage (3). J'ai créé, dans ma collection, pour ees mollusques la tribu des Isoglossata. La disposition des séries ou rangées varie ehez les divers mollusques; ainsi elles sont disposées sur une ligne droite: Planorlis contortus; ou en lignes courbes: Limax carinatus; à angle rentrant: Cacilianella acicula; ou saillant: Ancylus fluviatilis; à legères courbures ou sinuosités symétriques: Vitrina pellucida, Zonites striatulus, Zna lubrica, Helix obvoluta, etc.; ou bien en séries obliques qui des bords lu ruban lingual descendent vers le centre: Testacella, Daudebardia, etc.

(1) Quelques naturalistes ont regardé cet organe comme une måchoire inféricure.

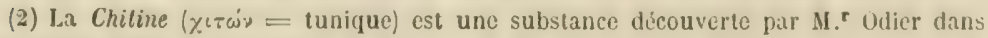
les parties solides et térumentaires tu corps et des membres des Insectes et des Crustacés, dans la composition des quelles elle entre pour un quart. On l'ohtient en traitant ces deux parties par la potasse a chaud qui ne fait que la degager, sans la dissoudre, des autres substances animales avec lesquelles elle se trouve mélé. Eile offre pour caractere d'être soluble dans l'acide sulphuriq̨ue a clıaud, de ne point jaunir dans l'acide nitrique, de brûler sans se fontre, en laissant un charbon qui conserve la forme de l'organe brûlé, enfin de ne pas cuntenir de l'acule. l'ar ec dernier caractere li Chitine se rapproche des sulsstances végétales.

(3) $P$ avement, Macdonals. 
Les dents linguales sont simples, bifides, trifites et même quelquefois quadrifides, à pointes inégales, et dont les externes sont presque toujours les plus basses. Souvent les pointes obtuses sont surmontées d'un appendicule ou dent conique, courte et forte: Limax, Vitrina, Zonites, Zua, etc.

Chaque série transversale est composée d'une dent médiane ou centrale, et de plusieures dents latérales; souvent les dents latérales allant vers les côtés externes de la langue changent plus ou moins de forme; si elles sont tricuspides, ou bicuspides, les dentelures ou pointes latérales à la pointe élevée centrale, et plus encore celle du côté interne, s'oblitèrent; la pointe centrale se transforme elle aussi, de manière que les dents marginales de la plaque linguale sont quelquefois remplacées par de vraies pointes élevées, simples, fortes, cunéiformes ou aculeiformes, et plus ou moins recourbées; c'est ce qui a lieu ordinairement chez les Oxynatha.

Dent centrale - a) simple. Cette dent, ou papille, est quelquefois cn forme de cône plus ou moins obtus: Vaginulus, Philomycus, Amphipeplea, Limncea, etc.; ou en forme d'ergot: Borus; longue, étroite, à sommet lanceolé, obtus: Achatina fulica; petite, sub-carrée: Bulimus cinnamomeo-lineatus; courte, très petite: Stenogyra (Bul.) decollata et octona; large, sub-carrée, obtuse: Bostryx (1); large, allongée, tronquée, sub-arrondie au sommet: Ariophanta retrorsa (sinistr.).

b) bicuspide ou bifide: pointes larges, sub-quadrangulaires: Orthalicus (Bul.) gallina sultana; plus ou moins cunéiformes: Camptonyx, Planorbis.

c) trieuspide ou trifide: pointes mamelonnées, plus ou moins obtuses ou cunéiformes, la médiane ordinairement plus élevée et robuste: Arion, Limax, Tennentia, Grotis (2), Vitrina, Simpulopsis, Succinea, Zonites, Helix (stricto sensu), Zua lubrica, etc. (3).

d) $q$ u a drifi d e: pointes arrondies, les deux médianes égales, les deux externes plus basses: Pellicula.

Dents latérales - a) s imples: fortes, un peu courbées, pointues, en forme d'ergot: Borus; médiocres, coniques, obtuses: Vaginulus, Plilomycus; larges, sub-carrées: Bostryx.

b) bicuspides ou bifides: pointes sub-carrées, ou cuneiformes, sub-arrondies ou mamellonnées, etc. ${ }_{i j}$ ia dentelure interne est presque toujours

(1) Bulimus, (Bostryx) solutus, Troschel-in: Zeitschr. fur Malakozool., 1847.

(2) Goeotis, Shuttleworth - Beiträge zur näheren Kenntniss der Land-und Süisswass. Moll. der Portorico; in: Mittheil. Naturf. Gesellsch. in Bern; märz, 185̆4. - M. ${ }^{\mathbf{r}}$ Fischer (in: Act. Soc. Linn. de Bordeaux, tom. XX, juin, 1855) donne ce genre comme synonyme de Peltella, Welb et Van Beneden.

(3) Les pointes ou dentelures de chaque côté de Ia pointe centrale, chez la Zuas ?ubrica et l'Helix nautiliformis sont très émoussées et reduites presque a néant. 


\section{$-116-$}

la plus longue: Vilrina, Simpulopsis, Succinea, Zonites, Helix (stricto sensu), Zua, Bulimus cinnamomeo-lineatus, Orthalicus, Achatina fulica, Camptonyx, Limncea, ete.; une pointe arrondie et l'autre courbée en forme de bes sur elle, et munie du côté interne de petites denticules: Bulimus lita; dentelures ou pointes égales, symétriques, obtuses: Tennentia; ou sub-arrondies: Ariophanta retrorsa.

c) trifides ou tricuspides: Arion, Limax, Grotis, Rumina (Bul.) decollata, Planorbis, etc.;

d) qua drifides: Amphipeplea.

Dents marginales - a) simples: courtes, coniques, très petites: Philomycus; cunciformes et presque droites: Camptonyx; allongées, fortes, plus ou moins subulées et récourbées: Limax, Greotis, Vitrina, Zonites.

b) bi c u spides: dentelure ou pointe interne longue, conique, un pen courbée; l'extérieure très petite, tuberculiforme: Planorlis carinatus; pointes inégales, obtuses: Bostryx; dentelures égales, symétriques, chaque pointe dle même courtement bifide: Ariophanta retrorsa.

c) tricuspides ou trifides: Simpulopsis; les deux pointes plus courtes d'un seul côté (l'externe): Bul. cinnamomeo-lineatus.

d) in ul tic uspides: larges, terminées à dentelures petites, uniformes: Amphipeplea.

Les denticules ou papilles linguales chez la Parmacella Deshayesii (1) sont réduites à de simples asperités ou denticulations aplaties, triangulaires. Le muscle semi-circulaire de la poche huccale n'est jamais en contact avec les aliments, et la plaque cornée ou linguale n'offre aucune trace de raisseaux, ni de nerfs. "Ce ne sont pas, observe M. ${ }^{r}$ Fischer, des organes de gustation, mais on peut les rapprocher des armures du palais de eertains poissons ". Cette singulière organisation est elle caractéristique et commune ì toutes les vraies Parmacelles (exelus.: Peltella et Parmarion)? - Chez les mollusques carnassiers, la dent on papille centrale n'existe pas; leur membrane linguale est garnie l'une grande quantité de spinules visibles même à l'œil nu, fortes, allongées, acérées, disposées en series ou rangées transversales et obliques formants un angle entrant médiane, mais désunies. Ces spinules sont coniques, légèrement couxbées, ì convexité dirigée en dedans, terminées à crochet, et avec un rentlement ou apophyse vers la moitié: Testacella, Glandina; ou bien elles sont aigiies, lisses, non crochues, et avec leur partie inférieure renflée: Daudebardia.

Pour désigner la disposition des dents ou papilles linguales de chaque rangée (2), il vient très '̀ propos d'user la formule dẹjả employéc pour repré-

(1) 1'. Fisther - Melunges de Conchyluolngie; in: Act. Soc. Linn. de Bordeaux, 1855.

(2) Cilierd, "n allemand. 
senter la dentition des animaux vertébrés. Ainsi, p. ex., la notation 55. 1.55 indique une série ou rangée de 55 dents ou papilles latérales de chaque côté de une dent ou papille centrale. Cependant, comm'il devient utile de tenir. compte quelquefois même du nombre des séries ou rangées, ainsi peut servir la notation formulée de cette manière: $\frac{57.1 .57}{100}$. Par cette notation j'aurai représenté le nombre (100) des rangées dentales de la langue de la Vitrina pellucida, et le nombre des dents médianes ou centrales (une) et latérales (37 et 37) de chaque série ou rangée; en total 7,500 papilles! Le nombre des dents ou papilles linguales de chaque série, de même que le nombre des séries ou rangées est très variable, et non seulement dans les divers genres, mais chez même les différentes espèces. En voici quelques exemples parmi les inoperculés (1): Succinea putris $\frac{59.1 .52}{50}$; Balia fragilis $\frac{20.1 .20}{130}$; Clausilia laminala $\frac{25.1 .25}{120}$; Claus. nigricans $\frac{20.1 .20}{90}$; Arion empiricorum $\frac{55.1 .55}{160} ;$ Helix cingulata, Var. Luganensis, Schintz; et $H$. nisoria, Rossm. (à peu près) $\frac{25.1 \text {. } 25}{80} ; H$. tigrina, Jan, de 80 à 90 rangées d'environ 60 papilles grosses et assez saillantes; $H$. aspersa $\frac{52.1 .52}{\mathbf{1 5 5}} ; H$. caperata, Montagu (d'Angleterre)

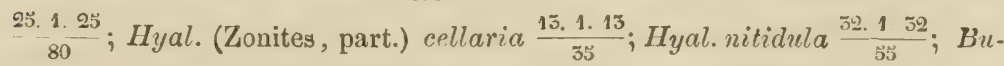
limus obscurus $\frac{27.1 .27}{120}$, ete.

\section{Quelques abservations sur les Vitrines.}

Nous avons déjà observé que les Vitrines possèdent une mâchoire lisse, arquée, et munie d'une forte saillie rostriforme sur le milieu du bord libre ou inférieur; et que leur langue est couverte de nombreuses rangées de denticules ou papilles dont la centrale est bifide, les marginales subulées ou aculeiformes. Dans mes diverses excursions malacologiques j'ai preparé avec soin et conservé dans l'alcool affaibli quelques exemplaires adultes de cinq espèces de Vitrines que j'ai pu trouver vivantes: Vitr. brevis, Fer.; Charpentieri, mihi (= nivalis, Charp.); diaphana, Drap.; major, Fér.; et pellucida, Müller. $J$ 'ai soumis quelques individus de ces Vitrines au scalpel de M. ${ }^{r}$ Sordelli, et voici les resultats des nos observations: Les mâchoires de la Vitrina Charpentieri, diaphana et pellucida présentent bien peu de différences; elles sont très arquées, larges, hautes, et un peu droites en dessus, ce qui leur donne un'apparence un peu sub-carrée. La mâchoire de la $V$. major est un

(1) Thomson Villiam: Remarks on the Dentition of British Pulmonifera; in: Annals and Mag. of nat. hist., London, febr. $1851 \Rightarrow$ Saint-Simon: Miscellances Malacol., 2. ${ }^{\mathrm{e}}$ décade; Toulouse, 18 ร̌. 
peu moins grande, elle est arrondie supérieurement, et à extremités plus attenuées, mais un peu obtuses. La mâchoire de ces quatre espèces est munie d'une saillie rostriforme très forte, en forme de cône renversé; un peu plus large, mais plus obtuse chez la V. Charpentieri. La partie supérieure de la mâchoire, appendice ou espèce de racine qui s'emplante dans les lèvres, est longue et très étroite chez la $\nabla$. major; un peu plus haute chez la Charpentieri, étroite et aussi longue que la mâchoire chez la diaphana, courte mais plus haute chez la pellucida. Mais la mâchoire de la $V$. brevis, de même que ses dents linguales, présentent des notables différences: la mâchoire est très large, et peu haute, et la saillie rostriforme n'est représentée que par une simple avancement au milieu du bord inféricur; elle est large et très obtuse; l'appendice, ou racine supérieure est arrondie, étroite et aussi longue que toute la mîchoire.

Les dents linguales de la $V$. Charpentieri, major, et pellucida ne sont guère différentes entr'elles, si ce n'est que chez la $V$. major elles sont un peu plus petites. Leur dent centrale est trieuspide, les dents latérales bicuspides, les marginales, ou spinules, sont hautes, aculeiformes, un peu obliques et inclinćes en dedans. La pointe ou dentelure médiane de la dent centrale, de même que la pointe interne, ou la plus grande', des dents latérales est très élevée (un peu moins chez la $V$. diaphana), mamelonnée, un peu enlargie au sommet et arrondie (1); le côté ou paroi interne des dents latérales est sensiblement sinué.

Chez la $V$. diaphana les pointes ou dentelures mineures de la dent centrale, sont presque réduites ì néant, et n'y sont tracées, pour ainsi dire, que par la sinuosité qui est de chaque côté de la grande pointe médiane; les denticules ou spinules marginales chez la $V$. diaphana sont plus obliquement inelinées.

Quant à la $V$. brevis, ce n'est pas seulement la dent centrale qui est tricuspide, mais un certain nombre aussi des dents latérales; la dentelure ou pointe principale est très élevée, mamelonnée, arrondie au sommet comme chez les autres espèces; mais les dentelures inférieures, sont tout à fait émoussées et arrondies de manière qu'on dirait que c'est une pointe solitaire qui s'élève du milieu d'une base large et sub-arrondie; cependant chez les jeunes individus, et tant plus chez le nouveau-nés, la dent centrale et plusieures des latérales sont distinetement tricuspides, à dentelure médiane plus élevée et peu obtuse; celle-ci dans le dent centrale s'enlargie un peu au sommet, et est termincée en pointe qui paraît triangulaire. Chez les adultes, après un certain nombre de dents obtusement tricuspides, celles-ci se changent ensuite en bicuspides, à peu près de la même forme que celles de la $\boldsymbol{V}$. dia-

(1) Tontes les grandes pointes, soit de Ia dent centrale que des latérales chez les Vitrines, sont surmonties de cette especce de appendicule conique obtus dont nous avons dejia parle, et qui existe aussi chez les Limax, Zoniles, Zua, etc. 
phana, mais à pointe ou dentelure extcrne plus émoussće, c'est à đire réduite à une élevation sub-arrondie, et à côté interne un peu sinué; bientôt cette élevation s'amoindrit elle aussi, la dentelure ou pointe principale s'amincit, la sinuosité interne devient plus sensible, et voilà les dents latérales changées en pointes marginales, remarquables par leur forme, car elles ne sont pas ni hautes, ni aculeiformes ou subuliformes comme chez les autres Vitrines et les Zonites, etc.; mais basses, larges, fortes, peu obliques et ì sommet assez obtus, on les dirait un quid intermediaire entre les dents et les spinules.

Lorsque nous avons parlé de la forme des dents ou papilles linguales, nous avons fait remarquer que les mollusques pourvus de dents médianes et latérales comme chez les phytivores, et de spinules marginales comme les carnassiers qui se nourrissent de proie morte, appartiennent presque tous aux Oxygnatha, e'est à dire à ces mollusques dont la mâchoire présente une saillie rostriforme au milieu du bord libre. Or, d'après l'inspection des organes de la mastication de la $V$. brevis, il parait qu'il y ait aussi un certain rapport entre la forme des spinules marginales et la forme de la mâchoire; c'est à dire que lorsque la saillie rostriforme de la mûchoire est peu prononcée et très obtuse, les dents ou spinules marginales sont elles aussi moins élevées, plus larges et plus obtuses, plus dentiformes que aculeiformes; le mollusque done plus phytivore que carnassier.

\section{Notes sur les genres Tennentia, Parmarion, Helicarion, et Camptonyx.}

Les observations anatomiques sur les Mollusques du Piémont nous ont nécessairement entrainés à parler aussi de plusieures espèces et même de quelques genres exotiques; quelques-uns de ces genres, d'autant plus interessants quant ils sont moins connus, méritent bien des notes illustratives. Quelques partisans trop zélés, peut-être, de l'unité de composition, pourront nous opposer un magistral: hic non est locus, et nous blâmer d'avoir trop hardiment sauté des rives du Pô et de la Trebbia à celles du Ganges, etc.; ils ont quelques raisons, eux, de s'écrier, mais le monde savant, au contraire, conviendra avec nous que toute occasion favorable est toujours opportune pour publier des faits interessants pour la science.

Tennentia, Iumbert - Ce genre, d'après ce qu'en dit M. ${ }^{r}$ Fischer, (in: Journ. Conch.; Paris, 1863) doit être considéré comme un double emploi du genre Parmarion; cependant M. ${ }^{\mathrm{r}}$ Humbert (Études sur quelques Moll. terr. nouv. ou peu connus; in: Mém. Soc. Phys. et Hist. nat. de Genève, 1863) fait ressortir quelques notables différenees par lesquelles on ne saurait si facilement admettre l'identité du genre Trnnentia avec le Parmarion. Quant à nous, nous voudrions ajouter, que s'il y a un quelque doute sur l'ammissibilité du genre Tennentia, ce n'est pas à cause de sa ressemblance avec le genre Parmarion, 
du quel il se distingue, au contraire, par plusieurs caractères; mais bien plutôt par sa grande affinité avec le genre Maricella(1). La limacella de Tennentia Thucaitesii ne diffère que très peu de celle de Maricella Dussumieri; quant à l'animal, d'après les observations de M.r ${ }^{r}$ Iumbert, il se distinguerait de celui de Maricella par son manteau $\mathrm{ne}$ faisant pas saillie en avant sous forme d'un collier charnu, et n on fr a n g é sur les côtés. Il vient très à propos ici de parler d'un autre mollusque assez interessant, décourert par M. ${ }^{r}$ Morelet à l'île de Saint Miguel (Açores): Viquesnelia Atlantica, Morelet et Droü̈t (in: Notice sur l'Hist. nat. des Açores, ete. Paris, 1860, pag. 139, pl. 1, fig. 1.) Cette limace rapportée par MI.r Morelet et par quelques autres auteurs au genre Viquesnelia, s'en distingue an prémier abord par son pied qui n'est pas tronqué postérieurement comme celui de Maricella (ou Viquesnelia) et par l'absence du pore muqueux terminal; son ouverture respiratoire est située à la partie postérieure droite du manteau, comme chez le Limax; celle de Maricella, n'a pas été indiquée par M.r Gray, mais chez le genre Tennentia elle est au milieu du bord droit. C'est done évident que le mollusque de M.r. Morelet, quoique un peu allié de Maricella et de Tennentia par la forme de la limacelle, il doit néanmoins constituer un genre à part au quel nous donnerons bien volontier le nom de Plutonia, déjà opté et non adopté par MI. ${ }^{\mathrm{r}}$ Morelet même (2). Mais où placerons-nous la Viquesnelia lenticularis, Deshayes (non Fischer, nec Morelet)?... Ce genre assez problématique fût créé pour une espèce de rudiment ou osselet testacé fossile, trouvé par M. ${ }^{r}$ Viquesnel en Roumelie, et entassé par centaines (!) sur une surface de sept it huit centimètres carrés dans une couche fluviatile ou lacustre; et trouvé aussi par 1I. ${ }^{\mathrm{r}}$ d'Archiac dans le terrain nummulitique des Pyr'énées. Même en admettant (ce qui est bien donteux!) que cette petite pièce ait appartenue comme limacelle à un gastéropode terrestre, on ne saurait pas certes réunir ce rudiment testacé à quelque se soit des genres connus des Limaciens vivants. Un nouvel arrangement de ce petit group étant done necessaire, nous proposerions le suivant:

(1) Mariwlla, Gray - Cat. Pulmon. Brit. Mus. 1855 (= Viquesnelia, Fischer; non Deshayes nec Morelet).

(2) J'avais renfermé les animaux de celte espéce, nous écrivait M.r Arthur Morelet, lans un flacon qui s'est brisé, et dont les fragments on été dispersés pendant une de mes longues transverscies ...... Lorsque j'ai découvert ce mollusque, je ne connaissais pas te genre Viquesnelia, qui fût créé pendant mon absence, et je me proposais de l'appeler Plutonia en hommage des îles voleaniques parmi lesquelles je l'avais rencontré.... Vous me demandez si ma Víquesnelia allantica a le plan locomoteur tronqué avec un pore terminal, comme les Arion ... nullement: l'animal se termine à peu près comme lo Limax gagates. 
† Animal ì pied tronqué postérieurement, et ì pore muqueux terminal.

\section{Gen. MARI IELLA.}

Clypeidella, Valenciennes - Mss, ; non Clypidella, Swainson.

Maricella, Gray - Calal. Pulmon. Brilish. Mlls. 1855.

Viquesnelia, Fischer - in: Journ. Conch., Paris, 1857; non Viquesnelia, Deshayes; noc Morelet.

Espèce : Maricella Dussumieri, Gray - loco cilato.

(Clypeidella Dussumieri, Valenc. - Mss. = Viquesnclia Dussum., Fischer - loco cilato.)

Habit. Mahé (Seychelles).

\section{Gen. TENNENTIA.}

(Tennentia, Humbert - in: Revue et Mag. de Zool., Paris, 1868.)

Espèce: Tennentia Thwaitesii, Humb. - loco citato, pag. 11, pl. 17, fig. 1.

Habit. Région montagneuse de Ceylan; jardin botanique de Peradenia.

††. Animal à pied non tronqué et sans pore muqueux.

\section{Gen. PLUTONIA.}

(Viquesnelia, Morelet - Nolice sur l'Hist. nat. des Acores, etc. Paris, 1860; non Viquesnelia, Deshayes; nec Fischer.

Plutonia, Morelet - in litt.

Espèce: Plutonia atlantica.

(Viquesnelia allantica, Morelet et Drowët - loco cilato, pag. 159, pl. 1, fig. 1.

Habit. Ile de San-Miguel (Açores).

\section{Genre doutcux : VIQUESNELIA.}

(Viquesnelia, Deshayes - in: Journ. Conch., Paris, 1857; non Viquesnelia, Fischer; nec Morelet).

Espèce: Viquesnelia lenticularis, Desbayes - loco citato, pag. 295 , pl. VII , fig. 1417

Haвiт. Fossile à Balouk-keni en Roumélie (Viquesnel); les Pyrénées, " couche à Viquesnelia dans la partie inférieure du terrain nummulitique " (d'Archiac). 


\section{$-122$}

Parmarion, Fischer - Dans son excellente monographic de $l_{\mathfrak{a}}$ Parmacella Deshayesii, (in: Act. Soc. Linn. de Bordeaux, 1855) M.r Fischer donne une liste des Parmacellido divisées en trois genres: Parmacella, Cuvier; Peltella, Webb et Van Beneden; et Parmarion, Fischer. Si, comme le pense M. ${ }^{\mathrm{r}}$ Fischer, le genre Gacotis, Shuttl., est un double emploi du genre Peltella, nous avons déjà fait voir les différences de l'organe lingual de Gaotis et de Parmacella. Qunt au genre Parmarion il se distingue assez bien par l'ouverture dorsale sitúe au-dessus de la limacelle, et par le pore muqueux caudal qui n'existe pas chez les Parmacelles. Il paraît que M.r ${ }^{\mathrm{r}}$ Fischer n'ayant eu à sa disposition que les exemplaires dans l'alcool, de la collection de feu M. ${ }^{\mathbf{r}}$ de Férussac, n'ait pu examiner les organes maxillaires; cette lacune vient d'être comblée par M. ${ }^{r}$ le prof. ${ }^{r}$ Humbert, conservateur du Musée d'histoire naturelle de Genéve, qui s'occupe avec zèle de l'anatomie des mollusques. Dans ses: Études sur quelques Moll. terr. nouv. ou peu connus (in: Mém. Soc. Plyys. et Hist. nat. de Genéve, tom. XVII, I. ${ }^{\text {re }}$ partic, 1863), M. ${ }^{\mathrm{r}}$ Humbert a donné la description et les figures d'une espèce nouvelle de Parmarion ( $P$. pupillaris, II.; de Java), d'où il en résulte que la mâchoire de ce genre n'a aucun rapport avec celle des Parmacelles, mais est très semblable à la mâchoire du genre Limax.

Helicarion (Helixarion), Fírussac (二 Nanina, et Vitrina, quorund. anctor.) Ex: Helicarion Cuvieri, Fér. etc. des terres Australes. Peu distinct des Vitrines exotiques par sa coquille, ce genre en diffère par plusieurs caraetères zoologiques: troncature du pied, présence d'un pore muqueux, développement du manteau en avant et en arrière sur la coquille, etc. Mâchoire comme celle du Limax et du Zonites (Fischer - loco citato).

Camptonyx, Benson - L'unique espèce connuc (C. Theobaldi, Bens.), de ce genre interessant et curieux, vit sur les rochers de la haute montagne de Girnar dans le Kaltiawar (Ind. Orient.). L'animal de Camptonyx a quelques affinités avec celui d'Ancylus, Chilina et Scarabus: deux tentacules, courts, obtus, divergents; yeux sessiles sur le milieu de la base postero-supéricure des tentacules, etc. La coquille est pileiforme, conique-oblique, bruneroussâtre; spire de $1 \frac{1}{2}$ tours, à sommet libre, subspiral, obliquement courbé; le côté droit du test présente une saillie dorsale, en forme de carène obtuse, qui du sommet arrive jusqu'au bord de l'ouverture, et corresponde intérieurement à une gouttière syphonale.

$$
\text { Ilauteur, } 4 \text { 1/2 millimètres; Diamètre grand, 8; petit, } 6 \text { mill. }
$$

Le Camptonyx, par la forme de sa coquille, ne peut être rapprochó que de Otina et de Ancylus; mais on le distinguera de la première: à sa gouttière syphonale, et par l'habitat (l'Otina vit sur les rochers au bord de la mer); de l'Ancylus il s'éloigne encore plus par la forme des dents linguales, par 
la mâchoire solitaire et non papillifère (les Ancyles possèdent trois mâchoires, et papillifères), et partecipant à la fois de celles de Zonites et de Succinea; enfin par la forme même de la coquille qui ressemble plutôt à un Pileopsis que à un Ancyle.

Note sur les Proserpinides et sur les genre Amphibola

Les Proserpinides (Proserpina, Gray; et Ceres, Gray) forment un'exception dans l'ordre des Pulmonés Inoperculés, comme l'Amphibola Schumacher, dans celui des Operculés. L'Amphibola, qui par son habitat et par sa coquille est un peu allié des Ampullaires, est un mollusque operculé (1), mais avec les caractères des Inoperculés Adelopneumones Isoglosses (androgyne; orifice respiratoire muni de sphincter; plaque linguale construite sur un plan uniforme, à rangées très nombreuses et horizontales).

Les Proserpinides, au contraire, sont des mollusques inoperculés (2) qui présentent les caractères des Pulmonés operculés, et se rapprochent, par la forme de leur coquille, aux Helicines (ordre des Phaneropneumones, tribu des Rhipidoglosses). (3).

\section{De l'organe de l'oüie chez les Mollusques.}

Niés d'abord, puis imparfaitement observés ces organes, ont été enfin bien reconnus et étudiés par Siebold, Pouchet et Van Beneden, ensuite par Eydoux et Souleyet, Laurent, Wagner, Moquin-Tandon, Claparède, Macdonald, Adolphe Schmidt. L'organe auditif des mollusques consiste en un petit sac (poche ou bourse auditive) rémpli d'un liquide aqueux tenant en suspension une ou plusieures concrétions (Otolithes) incolores et transparentes comme du verre, et de nature calcaire. Ces petites poches, car il y en a une de chaque côté, sont placées, chez tous les Gastéropodes, contre la partie postérieure, ou postero-inférieure de la première paire de ganglions sous-œesophagiens, aux quels sont unies par un pédoncule. Ce pédoncule est creux à l'intérieur dans la plupart des espèces terrestres et d'eau douce, mais chez quelques-unes, au contraire (Cyclostomus elegans, etc.) ce canal ou conduit interne n'existe pas (on verra ci-après à quelle fonction il est destiné). La forme des Otolithes est, le plus généralement ovoïde, mais il y en a d'arrondies, de fusiformes, ou paraissant composées de quatre pyramides, de trapezoïdales, etc. Quelquefois on observe une grande variation de formes dans une même espèce; ainsi p. ex. les Otolithes du Pomatias maculatus se présentent sous plus de dix formes différen-

(1) Tentacules nuls; yeux portés sur un lobe arrondi.

(2) Animal dioïque, orifice respiratoire large; deux tentacules, courts, subulés; youx sessiles, à la partie extérieure de la base des teutacules.

(3) Voir plus avant la note sur les Pulmonis operculés. 
tes: subcylindracée, parallèlipipède, pentagonale, trapezoïdale, ete. (1). Le nombre de ces concrétions varie de quatre seulement jusqu'à plusieures eentaines; il n'y a que peu de mollusques dont la poche ou capsule auditive contient une scule otolithe (Ex. Cyclost. elegans, C. sulcatus, C. Voltzianum, Truncatella lecvigata; Hydrocana Sirkii; Bythinia (Hydrobia) acuta, viridis, similis, etc.; Lithoglyphus fuscus et naticoides, etc. Chez la plus grande partie des Gastéropodes les otolithes sont trìs nombreuses, et varient selon l'espèce et l'âge des mollusques; leur nombre n'est pas toujours en rapport avec les proportions de l'animal (2), et il arrive aussi quelquefois que les otolithes contenues dans l'une des poches sont moins grosses et moins nombreuses de celles de l'autre poche.

La poche auditive grossit à mésure que le nombre des concrétions s'augmente. Libres et isolées vers la paroi de la poche, les Otolithes sont groupées sous la forme de très petites granulations (Otoconia), ovoüdes, arroudies ou irrégulières, au centre de la poche même. Ces concrétions libres, suspendues dans le liquide des poches auditives, comme nous avous dit, sont mises continuellement en mouvement par des cils vibratiles, très petits, qui tapissent la face interne de la poche; les otolithes se dirigent de la périphérie au centre où sont les corpuscules pierreus accumulés et serrés les uns contre les autres; mais entrainées le otolithes vers la masse centrale, elles sont bientôt répous. sées, et dès qu'elles touchent à peine la paroi, elles se rejettent de nouveau vers le centre, et ainsi de suite; elles sont soumises à un mouvement continuel d'impulsion et de répulsion. M.r. Claparède (3) a bien observé les cils vibratiles et le tremblement des otolithes dans la capsule ou poche auditive du Pomatias maculatus: la plus légère compression pousse ces petites corpuscules (de forme cylindrique), l'un après l'autre, dans le canal ou conduit interne du pédoncule dont le diamètre égale celui des otolithes.

Quant ì l'organe transmissif, ou conduit des undes sonores, on ne sait pas encore s'il y en existe. "Est-ce que, se demande ici Moquin-Tandon, le son arrive à la poche auditive par la cavité buccale, ou bien se propage-t-il simplement à travers les tissus? n Et à quel dégré existe-t-elle la faculté auditive chez les mollusques? D'après les observations de Moquin-Tandon et de M. ${ }^{r}$ Baudon, les mollusques aquatiques, et surtout les acéphales, paraissent bien

(1) Ad. Schmidt - Gehörorgan des mollusken, in: Beiträge zur Malakologie, etc. Berlin, 1857.

(2) Plusieures centaines d'otolithes dans l'Arion rufus, to Limax maximus, etc.; un peu plus de 100 dans la Succinca putris, l'Helix rotundata, arbustorum, pomalia, etc.; á peu pres le même nombre dans la Limna stagnalis; 25 à 30 dans le: Carychium minimum, le Melanopsis Dufourii, elc.; 40 à 50 dans la Physa fontinalis : 70 dans le Pomatias maculatus, etc.

(3) Cyclostom. elegantis anatome; Dissert. inaugur., ctc. Berlin, 1857. 
plus sensibles que les terrestres. Mais aussi chez les aquatiques la fonction auditive n'a pas la même intensité. Nous étions un jour occupés à examiner les mouvements dans l'eau de quelques Bythinia fuminensis et d'une B. (Pyrgula) annulata qui se promenaient sur le fond du petit vase où nous les avions placées; ] a $B$. fluminensis était presque insensible même à un fort bruit, tandis que l'animal de la $B$. annulata, timide et paresseux, rentrait presque entièrement sont pied dans la coquille au simple bruit de notre voix.

(2) "L'on donne, comme l'un des caractères du genre Arion la situation antérieure de l'orifice pulmonaire sur la cuirasse; j'ai constaté souvent le contraire. La présence de la glande caudale a une valeur beaucoup plus importante et invariable. Cette glande ne constitue pas le seul organe sécréteur du liquide poisseus qui lubréfie la peau des limaciens; ce tissu renferme dans son épaisseur une grande quantité de glandules microscopiques dont les conduits aboutissent au dehors et lâchent la liqueur qu'ils contiennent pendant les efforts de la contraction "(Baudon: Nouv. Catal. Moll. de l'Oise, 1862).

Il faut se rappeler aussi que la mâchoire des Arion est bien différente de celle des Limaces.

(3) Cuirasse recouvrant de petites granulations calcaires, inégales, isolées, sans traces de limacelle. (Note oubliée dans le texte).

(1) Moi, et M.r le doct. Baudon, nous avons tenté en vain de conserver en place sur l'Arion rufus la coloration du corps. M. ${ }^{r}$ Baudon essaya aussi dans l'huile et la benzine, etc.; mais rien n'a pu empêcher cette matière d'être entraînée avec le mucus. La coloration de certaines espèces d'Arions n'est pas propre à la peau, c'est une substance particulière, une partie du mucus déposée à la surface et $\mathrm{y}$ adhérant à peine.

(5) Nous avons déjà observé que les Vitrines ont la mâchoire et les denticules marginales de leur langue semblables à celles des Zonites. Il parait que le besoin d'aliment est incessant chez elles, et que l'appetit ne leur faisse jamais défaut. Ces petits mollusques broutent les mousses et dissèquent les feuilles tombées et ramollies par l'humidité, en ne laissant que les fortes nervures; les substances végétales en decomposition sont les préferées. On ne doit pas conclure d'après cela que toutes les espèces de Vitrines sont exclusivement herbivores; au contraire il y en a quelqu'une qui, dans des circonstances favorables, est aussi bon carnassier, et se nourrit non seulement de proie morte, mais même de proie vivante et à tissu dur et résistant. Les expériences faites sur la Vitr. pcllucida, Müller, par mon honorable ami M. ${ }^{\mathrm{r}}$ le doct. Baudon de Mouy-Oise (Nouveau Catal. des Mcll. de l'Oise, 1862), sont assez ctrieuses et interessantes; je laisserai done parler M. ${ }^{r}$ Baudon même.

“ J'avais, dit-il, mis un gran nombre d'individus sous une cloche avec d'autres mollusques, en ayant eu soin d'y placer les roches, feuilles, et mousses sous lesquelles ils vivaient. Liune de ces vitrines s'introduisit dans les tit d'un 
Zonites cellavius et le dévora; deux Ilel. candidissima tuées par leurs molsures, furent dérorées aussi en deux jour's .... Je plaçai alors sous ma cloche un fragment de mouton eru, de la grosseur d'une noisette; cinq minutes après les Vitrines les plus voisines quittent leur place, quoiqu'étant fort occupées à manger des feuilles; .... de toutes parts je vois arriver mes Vitrines, elles quittent les trous où elles étaient cachées ..... c'est un rendezvous gẻnéral; celle qui suçait les restes du $Z$. cellarius abbandonne sa vietime pour cette proie d'un nouveau goût. J'ai remarqué à ec moment une véritable expression dans leurs tentacules; ils ne tîtonnent plus une fois que le ganglion nerveux a perçu l'odeur. On croirait que la vie est concentrée dans ces tiges délicates! On remarque bien de l'animation dans l'oreille de certains êtres d'une organisation supérieure; pourquoi n'en trouverait-on pas, quoique ì un moindre dégré, dans le tentacule d'un mollusque, siège principal de sa plıysionomie! Les entailles de la mâchoire de cette Vitrine, sur la chair ferme et fraîche, sont régulières et circulaires. Elle met six heures pour creuser trois millimètres de profondeur, mais elle se fatigue vite et ne peut déchirer longtemps; elle ronge les portions museulaires et abbandonne la graisse et les aponévroses; cette nourriture substantielle étant prise, elle reste plusieurs jours saus manger, et son activité est moins grande qu'au moment où son alimentation est végétale. Les vitrines ont besoin d'humidité; $j$ 'avais soin de verser de l'eau dans le plateau; l'evaporation s'etait opérée parce que cet appareil était dans une pièce assez chaude, et la vapeur condensée couvrait les parois internes de la cloche; les vitrines sortaient alors pour lécher les gouttelettes, puis regagnaient leurs trous. n Jusqu'ici M. ${ }^{r}$ Baudon.

De mon côté j’ai essayé quelques expériences sur la Vitr. Urevis, Fér. Avant la fin de novembre je quittais la ville pour aller à la chasse des petits mollusques sur les coteaux et les rivages en pente douce, ombragés et humides de la Val-d'Olona, à six lieues au nord de Milan. $Y$ ayant recueilli quelques individus de Vitr. Urevis, ¿̀ mon retour, après deux jours d'absence, je plaçai six de ces vitrines dans un pot à fleurs commun en brique, assez grand, rempli de terre jusqu'à la moitié, et surmonté d'une cloche, ayant eu soin d'y laisser passage libre à l'air; je n'ai pas oublié de parsémer le terrain de débris de tuiles et de vieux mortier, de feuilles pourries et de feuilles de choux tranchées et arrosées d'eau fraîche, de petits morceaux de boeuf, de porc et de mouton crus, enfin j'y ai ajouté deux Helices ciliata, une II. nautiliformis et une $H$. incarnata vivantes. Nies vitrines n'avaient donc pas à se plaindre d'une captivité si adoncie qui pouvait bien leur faire presque oublier, si cela est possible, l'air natal des leurs coteaux. Je m'attendais d'un moment à l'autre de voir mes petites bêtes se diriger du côté de la viancle, ou bien attaquer les autres mollusques, mais rien de tout cela; au contraire elles sont restées cachées pendant trois jours, et je pensais qu'elles étaient mortes de chagrin, 
ou enfoncées dans la terre; mais au bout de ce temps-là, voila mes vitrines paraître assez gaies et se promener sur les choux. Je placais alors mon pot à fleurs dans une chambre plus chaude (9 à 10 centigr.). Deux vitrines succombèrent après six ou sept jours de captivité; les autres (quatre) se conservaient en bon état; elles se laissaient voir quelquefois, et bien rarement montaient sur les parois de la cloche; elles rongeaient les choux (que j'avais soin de changer souvent et d'arroser d'eau fraîche une fois par jour, de même que les pierres et la terre), puis regaguaient paisiblement leur retraite; mais elles n'ont jamais touché ni à la chair fraîche, ni à celle déjà un peu décomposée, et ont même laissé vivre en paix leurs compagnons de exptivité (1).

Deux vitrines se sont accouplées le 14 décembre; je ne sais pas si cela ait eu lieu aussi chez les autres, et si plus tôt, ou plus tard. Le 28 décembre j'ai vu des œufs deposés sur une feuille de chou, mais ils ont disparus quelques heures après, et je pense qu'ils auront été cachés sous les morceaux de briques ou plus probablement sous terre. Ces oufs étaient agglomérés ensemble, et de nature gélatineuse, d'un blanc laiteux et de la grosseur d'un grain de millet un peu oval. Après un'attente de trois mois, je n'esperais plus d'en voir l'éclosion; mais entre le sept et le huit d'avril, voilà sept petits se promener gais et voraces sur les briques et sur les feuilles; entre le quinze et le dix-huit du même mois, le nombre des nouveau-nés était de quinze ou seize. Leur accroissement s'opère lentement, de manière que deux mois après leur éclosion ils ne sont arrivés qu'au tiers à peine de l'entier développement. Les jeunes vitrines restent presque toujours cachées, et se promènent parmi les feuilles de laitue, qu'elles rongent avidement à préference des autres substances végétales.

L'animal des Vitrines à peine écloses, ou très-jeunes, lorsqu'il est en marche, c'est à dire en toute son extension, a une longueur de $2 \frac{1}{2}$ à $3^{\mathrm{mm}}$; et la coquille présente un seul tour, ou un tour et un quart de spire; mais les individus dont l'animal atteint quatre millimètres de longueur, ont déjà leur coquille composée de deux tours de spire. Chez les jeunes individus, l'animal est, en dessus et sur le devant, de couleur cendrée, avec une teinte roussâtre à la base des pédoncules, ou tentacules supérieurs, qui sont d'un cendré plus obscur. L'ouverture respiratoire est marquée elle aussi d'une tache ombrée demi-circulaire; le reste du corps est blanchâtre et demi-transparent. La coquille est transparente, et laisse voir la masse viscérale qui est d'un jaune ambré chez les individus qui n'ont pas mangé, et plus foncé et verdâtre après leur repas; on y aperçoit aussi bien marquée une petite bande transversale (portion du canal digérent qui correspond à l'estomac).

(1) Les notables difiérences dans les organes maxillaires et linguales de cette espéce, expliquent assez bien pouirquoi elle préfẻre exclusivement les substauces végétales (Voir la note: observations sur le Vitrincs). 
Des individus qui composaient la petite colonie du novembre, huit ont succombés ì des époques différentes, c'est ì dire, deux vitrines peu de jour's après leur reclusion, deux en février, un autre avec une des $I I$. ciliata aux premiers d'avril; la dernière vitrine, de même que l'autre $H$. ciliata, aux premiers de mai. La coquille des Vitrines, quinze ou vingt jours avant leur mort perd la transparence et devient opaque et blanchâtre; cet état, que nous appellerons état de vieillesse, varie de vingt jours jusqu'au delì de trente. L'II. nautiliformis était vivante encore en avril, lorsque nous en sommes servi pour l'anatomie; l'H. incarnata suecomba après sept mois de captivité; dès les premiers moments de sa nouvelle demeure elle fixa son séjour sur les parois vitreuses de la cloche, tout près des petites ouvertures, pour jouir de l'air et de la lumière; elle ne quittait sa place que pour descendre quelques moments pendant la nuit ì ronger un peu les choux ou les laitues. J'ai constaté qu'une seule vitrine adulte unangeait elle seule autant que les autres quatres mollusques du genre IIelix; les petites vitrines sont encore plus voraces. Les quelques observations que nous avons ici exposées sur le régime alimentaire de la Vitr. Urevis, servent ì nous persuader une fois encore de plus, que dans une science toute d'observation telle que la Malacologie, on ne doit pas trop se fier aux analogies, et tant moins, d'après un fait spécial, formuler une loi d'application générale.

Puisque nous arons eausé jusqu'ici sur les Vitrines, il ne sera pas inutile d'ajouter quelques mots sur le meilleur procédé pour nettoyer les Vitrines, c'est ì clire pour enlever à l'animal le fragile têt qui y est adbérent. Quelques conchyliologistes les laissent pourrir dans l'eau froide; d'autres les jettent dans l'eau bouillante, d'autres enfin dans l'alcool. Ces procédés ont tous leurs inconvénients, et d'albord: si l'animal pourri, la coquille subit elle anssi un'alteration clans la couleur, la transparence, ete.; si l'alcool est trop fort, ou non pur, chose frequente à nos jours, il gâte rapidement le têt des Vitrines non seulement, mais la coquille même plus solicle de Suecinées, des Zonites, etc. L'eau bouillante est peut-être à préferer, mais le procédé meillour et le plus simple et facile e'est de placer les Vitrines sur une bande de papier et de renfermer ce papier dans un flacon queleonque (placé horizontalement, ì fin que les Vitrines ne tombent pas) dans lequel on ait préablement versé quelques gouttes (p. ex. 12 à 20) de benzine. Au bout de cinq ou six minutes, ì proportion de la grosseur de l'animal ou du degré de tenacité de sà vie, (lia Vitr. brevis est asphyxiće un peu plus vite que la Vitr. pellucidlu), on rétire le papier avee les Vitrines mortes; il suffit alors de fixer avec une épingle l'animal antérieurement, et avec un'autre épingle soulever transversalement la coquille qui se détache avee facilité, et qui est très polie et luisante.

(6) Denys de Montfort dans sa Conchylivlorjir Systimatique (IS10), créa le gemre Zonites pour quelques espèces hu geure Helix ì coquille mince, ì cou- 
leur d'arçoise, à péristome simple et plus ou moins tranchant. La diagnose de l'animal est venue plus tard confirmer cette division, qui n'avait été faite que d'après les caractères de la coquille, et à en augmenter même le nombre des espèces. Le Zonités diffèrent des Helices : - 1. ${ }^{\circ}$ par leur mâchoire simple, arquée, sans côtes ni dents, et à bord inférieur rostriforme au milieu (comme celle des Limaces; tandis que la mâchoire des Helices est ridée, ou à côtes et à marge denticulée, mais sans saillie rostriforme, comme celle des Arion) - 2. ${ }^{\circ}$ par la position de l'orifice sexuel placé (à droit) vers la partie moyenne ou la base du cou - $3 .^{\circ}$ par l'absence du dard et des vésicules muqueuses ou vermiformes $-4 .^{\circ}$ Les Zonites se nourrissent de proie morte, ainsi les denticulations ou papilles de leur langue sont tricuspides au centre comme celles des mollusques pulmonés inoperculés herbivores (Helix, etc.); et sont aculeiformes sur les côtés à peu près comme celles des pulmonés carnassiers (Glandina, Testacella, etc.) (1). La nature et la forme de la coquille, chez les Zonites, est très variable; ainsi p. ex. entrent à former ce genre l'H. cellaria, MülLER, sans dents; l'H. labirynthica, $\mathrm{S}_{A Y}$, avec des dents ou lamelles prolongées en dedans de l'ouverture; l'H. candidissima, DRAP. à têt épais et calcaire! Les progrés des études malacologiques feront subir, sans doute, des nouvelles modifications à ce genre; et déjà plusieures divisions viennent d'être adoptées, et on a créé même des genres nouveaux: Gen: Hyalina, pour les espèces du group de l'H. cellaria; Gen: Leucochroa, pour l'H. candidissima et ses affines; Gen: Zonites (stricto sensu) pour les Zonites à pore muqueux caudal, etc. Tout en tributant les plus justes éloges a ces infatigables illustrateurs de la malacologie, nous ne pouvons pas, cependant, nous ranger entièrement à leur manière de voir; car enfin plusieurs caractères ne sont pas exclusifs, et il y a souvent de passages de l'un à l'autre; l'absence du flagellum (p. ex.) n'est pas exclusive au group du Zonites olivetorum (Mesomphix), mais quelqques espèces aussi du group des Hyalines manquent de flagellum; si le Zon. candidissimus, à la place des vésicules muqueuses, présente un corps ou glande globuleuse, ovoïde, pédonculée, nous voyons aussi que chez quelques autres espèces du même group ( $H$. bretica, Rossm.; H. cariosula, Мıсн.) ce corps glanduleux n'existe déjà plus. Au contraire il y a quelques espèces appartenantes à d'autres sections du Zonites, chez lesquelles les vésicules muqueuses sont représentées par un enduit glanduleux plus ou moins distinct. Je pense done que l'on pourrait, pour le moment, modifier de la manière suivante la division des Zonites:

\section{Gen. ZONITES, Montfort:}

Animal: maxilla simplex, arcuata, non sulcata nec dentata, margine inferiori acuto, medio rostriformiter prominente. Dentes linguales mediani et la- 
terales ut in Helice; marginales elongati, aculeiformes (2). Systema sexuale simplex; sagitta ejusque bursa, et folliculi mucosi desunt. Apertura respiratoriæ latere dextro supra collare, genitalis ad basin colli.

\section{$\div$ Porus mucosus, in apice pedis, nullus}

\section{Subgen. IIyalina, Gray}

Folliculi mucosi nulli, aut ipsosum loco adest stratum plus minusve distinctum, glandulosum, subovaturn.

A. Conulus, Moquin-Tandon - Folliculi mucosi omnino nulli; tentacula inferiora crassa (Exempl: Helix fulva, Müller; H. semen-lini, Moricand; ete.).

B. Euhyalina, Albers - Flagellum breve, crassum, interdum obsoletum, raro nullum (Ex. $H$. cellaria, Müller; $\boldsymbol{H}$. crystallina, Mïller; etc.).

C. Mesomphix, Rafinesque - Flagellum nullum (Ex. H. incerta, Drap. H. olivetorum, Grmelin; etc.).

D. Gastrodonta, Albers - Apertura, basi, dentibus pliciformibus, marginem non attingentibus munita (Ex. H. interna, Say; H. labirynthica; Say; etc.).

Subgen. Macrocyclis, Beck.

Testa tenuis, peristoma subincrassatum, vix expansiusculum; maxilla arcuata, medio rostrata, tota costulis confertis, marginem non attingentibus; exarata (Ex. H. concava, Say; H. laxata, Fér.; etc.).

Subgen. Leucochroa, Albers. (Calcarina, Moq.-Tand.)

Systema sexuale sub-simplex; flagellum liberum, subulatum; folliculorum mucosorum loco adest interdum corpus granulosum, magnum, pedunculatum. 'Testa crassa, calcarea (Ex. H. candidissima, Drap.; H. cariosa, Olivier ; H. boetica, Rossmässler; ete.).

t+ Porus mucosus, in apice perlis.

Subgen. Zonitos, (stricto sensu).

Porus untcosus oblongus in apice pedis. Palpi labiales distincti. Folliculorum mucosorum loco adest stratum distinctum, truncato-ovoïdeum.

(i) Quand on aura cxamine l'animal, il est bien probahle que plusieures especers de Helices el de Nanines devront passer dans le genre Zonites. 
A. Egopis, Fitzinger - Palpi labiales parvi; porus mucosus mediocris (Ex. H. verticillus, Fér.; $H$. algira, Linné; $H$. gemonensis, Fér.; etc.).

B. Moreletia, L. Pfeiffer - Tentacula majora, seu peduncula oculigera, conica, apice inflato, ovoïdeo; palpi labiales majores, trigeni; porus mucosus amplus. (Ex. H. euryomphala, Pfr.).

(7) Les mollusques terrestres et fluviatiles operculés (à l'exception de l'Amghibola) (1) sont pourvus d'un orifice respiratoire large, et situé à la partie supérieure et postérieure du con, sans sphincter spécial, ou avec un sphincter rudimentaire.

Les operculés terrestrcs ou pulmonés sont diöques, et appartiennent à l'ordre des Phaneropneumones. Leur poche ou cavité respiratoire est tapissée d'un réseau vasculaire apparent, pour la respiration aérienne, et possède quelques rudiments de lamelles branchiales. Les operculés aquatiques sont presque tous dioïques, quelqu'un seulement androgyne (Valvata). Destinés à vivre dans l'eau, leur appareil respiratoire est branchial, composé de rides, de filaments, ou bien de lamelles sous de formes diverses. L'organisation maxillaire et linguale des operculés est bien différente de celle des inoperculés. Il n'y a que un petit nombre d'operculés pourvus de mâchoires; ces pièces ont beaucoup d'analogie avec les mâchoires latérales de certains mollusques inoperculés (Limnøa, etc.), et sont placées verticalement et latéralement, une à droite, l'autre à gauche de la cavité buccale (Vivipara $=$ Paludina, stricto sensu); quelquefois existe aussi une troisième mâchoire supérieure tout à fait rudimentaire, ou punctiforme (Valvata); ou bien au contraire les deux mâchoires sont placées horizontalement, l'une dessus, l'autre dessous, larges, à bord libre largement crénelé (Neritina).

Chez la plupart des operculés existent deux lames chitineuses, très minces, cartilagineuses, situées l'une à droite, l'autre à gauche de l'extrémité antérieure de la langue; ces cartilages sont formés de nombreuses petites lamelles hexagonales ou polygonales, et sont composés de deux pièces; l'une, antérieure, très grande, est reçue dans un sillon transversal de la marge antérieure d'uue autre petite pièce postérieure supplementaire; la marge extérieure du plus grand cartilage est, ordinairement, plus robuste; la marge intérieure est souvent sinuée (Cyclostomus, Pomatias, etc.), et y sont attachés les muscles de la langue. Ces pièces ou cartilages, auxquelles on a quelquefois donné improprement le nom de mâchoires, quoique subsidiaires à la langue dans ses fonctions ingestives des aliments, elles ne sont pas destinées cependant à faire l'office de mâchoires, et ne peuvent pas servir à cet usage par leur position et le peu de solidité.

Les papilles ou dents linguales offrent, elles aussi, des notables différences.

(1) Voir ci-dessus la note sur les Proserpinides et sur le genre Amphibola. 
Le nombre des séries ou rangées horizontales est variable, comme chez les mollusques inoperculés; ainsi pour citer quelques exemples parmi les operculés pulmonés: la langue du Pomatias maculatus porte, à peu près, 175 rangées ou 1225 dents; le Cyclostomus elegans 86 à 106 rangées; le Chondropoma Poeyanum 123; la Trochatella chrysostoma 51 ; la Tr. chrysochasma 60; I'Hydroccena Cattaroensis 50; la Truncatella Caribaensis 60; etc.

La forme des papilles ou dents de la langue, leur nombre et arrangement n'ont rien affaire avec les dents linguales des mollusques inoperculés. Elles sont recourbées en dedaus au sommet, et denticulées; chaque série ou rangée se compase d'une dent centrale ou médiane, ordinairement conique, ovale, ou sub-carrée, à sommet courbé en dedans; et de trois ou quatre dents latérales de chaque côté, placées transversalement, et à pointes ou courbures convergentes vers la dent médiane. D'après cette disposition qui rappelle un peu la forme de la eage thoracique des animaux vertébrés, les malacologistes ont appelé rachis la série longitudinale des dents centrales, qui représente comme une colonne vertébrale, et pleurce les dents latérales analogues aux côtes des vertébrés.

Nous ne parlerons ici un peu diffusément que des dents linguales des $O_{p}$ erculés pulmonés.

Tænioglossata - Représentés par la formule générale: 3. 1. 3.

Trois dents latérales de chaque côté de la dent centrale ou médiane; troisième dent latérale, ou externe, semblable aux deux internes; ou bien remplacée par une grande lame sub-triangulaire, à marge antérieure recourbée en dedans et pectiniforme.

A. Simples (1). - Dent centrale allongée, ou sub-carrée, recourbée en une seule pointe, ou multicuspide; dents latérales recourbées, uniformes, simples ou multicuspides.

a). Dents simples; centrale allongée, fusiforme, étroite, recourbée en dedans, à pointe courte, déprimée, simple, obtuse; $1 .{ }^{e}$ et $2{ }^{\circ}$ latérales un peu enlargies, ou renflées à la base, répliées vers le bord exterieur du ruban

(1) Cette premierc suction des Tonioglosses comprend une partie de la grande famille des Cyclostomes (Fam. Pomatiacea et Cyclotacea, Truschel - Gebiss der Schnecken, etc. 1, 1856, Berlin), et la famille des Truncatelles. Les Cyclostomes, proprement dits, appartenants aux Tænioglosses simples (Cyclotacea, Troschel) se distinguent aussi par la forme de leur opercule qui est arctispiral, c'est à dire à spire composée de nombreux tours. Entrent dans celte division les suivantes sub-familles de M. ${ }^{r}$ L. Pfeiffer (Monogr. Pneumonopomorum vie, etc.; Supplem. 1um, Cassel, 1858): Subfam. C y c lo. t a c e a (Gen. Cyclotus, Guilding; Opisthoporus, Benson; Pterocyclos, Benson; Alycneus, Gray); Subfam. Cyclophorea (Gen. Craspedopoma, Pfeiffer; Aulopoma, Troschel: Cyclophorus Monifort; Leptopoma, Pfeiffer: Dermatocera, H. el A. Adams), Subfam. Pup ine a fien. Megalomastoma, Guilding; Cataulus, Pfr.; Rophaulus, Pfr.; 
lingual en crochet peu courbé, fort et obtus; $3 .^{e}$ latérale ou marginale très petite: Pomatias (maculatus, patulus, scalarinus (1)).

b). Centrale large, à sommet recourbé et à cinq dentelures; latérales larges et hautes, recourbées, trifides: Cyclotus (substriatus, Sow.), Craspedopoma (lucidum, Lowe); ou avec la dent centrale tricuspide: Cyclophorus (aquila, Sow.); Registoma (grande, GraY; = Pupina Nunezii, Sow.).

c). Centrale conique, à pointe recourbée, portant vers la moitié un'élevation, ou arête horizontale formée de très petites denticules; $10^{\complement}$ dent latérale très large, quadrieuspide; $2{ }^{e}$ étroite, multicuspide; $3 .^{e}$ étroite simple: Truncatella (Caribæensis).

B. Pseudo-rhipidoglossata-Dent centrale sub-arrondie, recourbée, à une seule pointe, ou multicuspide; $1 .^{\mathrm{e}}$ dent latéral large; $3 .^{\mathrm{e}}$ ou marginale, représentée par une grande lame sub-triangulaire, à bord antérieur recourbé, multidenticulé, ou bien pectiniforme et à pointes ou dentelures cro. chues ou hamiformes.

a). Dent centrale ou médiane large, ovale ou ovalaire, à cinq dentelures; 1. e dent latérale grande, large, quadrifide ou quinquefide, à dentelure ou pointe du milieu très grande; $2 .^{\circ}$ latérale à marge supérieure recourbée quinquecuspide; $3 .^{\mathrm{e}}$ latérale, ou grande lame marginale, à bord antérieur recourbé, multidenticulé: Ericia (elegans, MüLLER; costulatus, ZIEGLER (2)); Leonia (mamillaris, LAMK.).

b). Semblables aux précedentes, mais la dent centrale est plus petite;

Streptalus (?), Benson; Pupinella, Gray; Pupina, Vignard; Regisfoma, Hasselt; Callia, Gray).

La 2. ${ }^{\text {e }}$ section des Tanioglosses nous la désignons sous l'appellation de PserdoRhipidoglossata, par la configuration des grandes lames linguales extérieures imilant celles des vrais Rhipidoglosses; et comprend la famille Cyclostomacea de M.r Troschel. Les Cyclustomes de cette section sont pourvus d'un opercule paucispiral; et corrispondent à la seconde parlie des sub-familles de M. ${ }^{\mathbf{r}}$ Pfeiffer $(0 p$. cit.; exceptées les suhf. Pomaliatea et Realia). - Subfam. L icine a (Gen. Jamaicia, C. B. Adams; Licina, Gray; Choanopoma, Pfr ; Ctenopoma, Shuttleworth; Adamsiella, Pfr.); Subf. Cy clost omea (Gen. Lithidion, Gray; Otopoma, Gray; Cyclostomus, Montfort, Tudora, Gray; Leonia, Gray); Subfam. Cistulea (Gen. Cistula, Gray; Chondropoma, Pfeiffer)

(1) Les dents linguales du Pom. naculalus, que j'ai fait examiner par M.r Sordelli, son très semblables à celles du $\boldsymbol{P}$. palulus figurées dans le classique ouvrago de M. Troschel (Gebiss der Schnecken; I, 1856; pl. IV, fig. 1).

(2) Les espèces du genre Cyclostomus (Cyclostoma (partim), LAMk.) comprises dans notre subdivision $-a-(C$. elegans, etc.), ont les dents linguales bien différentes do celles de notre subdivision - $c$; nous désignons donc notre première coupe sous l'appellation d'Ericia déjả imposée à ces espéces par Moquin-Tandon, d'après la forme de la coquille. Le nom de Cyclostomus 'stricto sensu) resterait donc à la dernière coupe. 


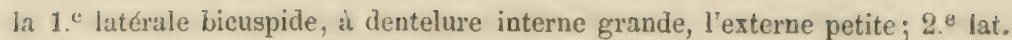
trìs étroite, quadricuspide: Chondropoma?, (Candeanum, ORBIGNY).

c). Dent centrale obtusement coniforme, à sommet recourbé, simple, plus ou moins aigu et plus ou moins large; $1 .^{\circ}$ lat. très large, récourbée, d̀ pointe solitaire; 2. ${ }^{\mathrm{e}}$ lat. étroite, à marge recourbée, bicuspide ou multicuspide; $3{ }^{\mathrm{e}}$ ou lame marginale, ì coupures plus longues, plus recourbées et crochues: Chondropoma (Poeyanum, Pfeiffer), Tudora (columna, Pfr.), Cyclostomus (Jayanus, Prr.; Banksianus, Sow.), Ctenopoma (rugulosum, Pfr.); Choanopoma) (Chittyi, Pfr.; scabriculum, PFr.), Cistula (catenata, Gould), etc.

Rhipidoglossata - Représentés par la formule générale: 00.4.1.4.00.

Une dent centrale, non recourbée; et quatre (rarement de moins ou de plus) latérales de chaque côté; et une très grande lame marginale formée de lamelles nombreuses et étroites, terminćes en pointe aculeiforme, hamiforme ou multifide, et toutes recourbées en dedans.

A. $H y d r o c a n i d a=00.1-1.1 .1-1.00$.

Dent centrale étroite, fusiforme, terminée en pointe pyramidale; $1 .^{\circ}$ dent latérale très rapprochée de la centrale, et presque de la même forme, mais a sommet obliquement plat; $2 .^{\mathrm{e}}$ et $3 .^{\mathrm{e}}$ latérales nulles, et un grand espace vide; 4. ${ }^{e}$ latérale étroite, subcylindrique, ì sommet pointu; grande lame externe ou marginale très longue et médiocrement large, composée d'un grand nombre de lamelles étroites et serrées, dont les premières ou postérieures sont cour. bées en dedans, à crochet simple; les antérieures ont leur marge supérieure récourbée vers les lamellez erochues, et tricuspide.

B. I e licinid $a=00.4 .1 .4 .00$.

Dent centrale petite, simple, imparfaitement sub-carréc allongée, à sommet plat, non recourbé. Dents $1 .^{\circ} 2 .^{\mathrm{e}}$ et $3 .^{\mathrm{C}}$ latérales petites, à marge recourbée vers le côté externe, simple ou denticulée; $4{ }^{e}$ latérale très grande et large; grande lame marginale formée de lamelles nombreuses, étroites et serrées, recourbées vers le centre, à pointes crochues, simples ou multicuspides.

a). Quatrième dent latérale très grande, étroite à la base, fortement enlargie au sommet, largement rebordée en dedans, à marge simple, rarement multidenticulée: Trochatella.

b). Quatrième latérale très grande, irrégulière, étroite, composée de deux pièces; celle du côté interne est à courbure convergente vers la dent centrale, multicuspide, et terminée à la base en forme de talon; la pièce externe sert de appui à la précedente, en même temps que à la grande lame marginale de la langue. Cette lame est formée de lamelles nombreuses, toutes bicuspides ou tricuspides: Helicina (stricto sensu).

C. Proserpinidce $=00.5 .1 .5 .00$. (Phaneropnenmones inoperculés) (1).

(1) Voir ci-dessus la nuto sur les Proserpinides. 
Dent centrale sub-carrée, large à la base, ensuite un peu rétrécie et comme étranglée, puis enlargie au sommet; $10^{\mathrm{e}}$ et $2 e^{\mathrm{e}}$ latérales à peu près de la même forme de la dent centrale, mais elles sont recourbées au sommet, et tricuspides; $3 .^{e}$ latérale allongée, très étroite, recourbée au sommet en deux petites denticules aigües peu apparentes; $40^{\mathrm{e}}$ et $5 .^{\mathrm{e}}$ latérales enormes, de forme irrégulière; la $4 .^{\mathrm{e}}$ est à peu près de la moitié plus petite que la $5 .^{\mathrm{e}}$, largement recourbée en dedans, à marge tricuspide ou quadricuspide, à pointe ou denticule interne (du côté de la dent centrale) très forte et large; la base est terminée en talon. La $5 .^{\mathrm{C}}$ dent latérale enorme, large et oblongue, rétrécie du côté extérieur de la langue, sub-carrée du côté central; repliée en dedans en un grand lobe sub-central, en forme de cône large et renversé, et dont la pointe arrondie descende en bas dépassant la base et formant une espèce de talon. Grande lame marginale formée de plusieures rangées de lamelles, les unes derrière les autres, longues, soudées à la moitié inférieure, libres supérieurement, rétrécies au sommet par une grande échancrure interne, et recourbées vers le centre de Ia langue; les premières tricuspides, bicuspides les autres: Ceres (C. Salleana, Gray).

(8) Plusieurs auteurs ont, plus ou moins, parlé du Cyclostoma elegans; les uns se sont limités à decrir la coquille, les autres les formes extérieures de l'animal, quelques-uns en ont étudié la merveilleuse structure interne. Troschel (1) a soigneusement décrit et figuré les dents ou papilles linguales. Moquin-Tandon (2), outre une bonne deseription des caractères extérieurs, a donné aussi quelques diagnoses des diverses parties de l'organisme de ce mollusque, et plus particulièrement du système viscéral nerveux, des organes de la reproduction, ete. Il paraît cependant que cet auteur n'ait pas fait usage du microscope composé, ainsi quelques-unes de ses observations sont reussies imparfaites, et quelques descriptions, pour ainsi dire, seulement ébauchées, Des nouveaux études anatomiques étaient done necessaires, et le mémoire de M. ${ }^{r}$ le Doct. ${ }^{r}$ Ed. Claparède de Genève (3) est venu combler ces lacunes. Comme cette brochure est assez rare, et même peu connue chez nous, ceux qui s'occupent d'anatomie malacologique auront pour agréable de voir ici reporté ce qu'il y a de nouveau ou de plus important dans la savante disertation du doct. ${ }^{r}$ genévois.

Système cutané. - L'épith élium de la peau (ou tunique dermo-musculaire) du C. elegans est dépourvu de cils vibratiles (4). Des concrétions

(1) Das Gebiss der Schnecken, Berlin; 1, 1856.

(2) Histoire natur. des Moll. terr. et puv. de France; Paris 1855. (Voyez: Ie Vol. 1 : études anatomiques et phisiol. des moll.; et Vol. II pag. 497).

(3) Cyclost. elegantis anatome - Dissertatio inauguralis; Berolini, 1857; avec deux planches gravées.

(4) Leydis, Siebold, etc. ont observé que le manteau de plusieurs céphalophores n'est pas protegé par un épithélium vibratil on ciliaire. 
calcaires sous la forme de petits grains blancs, agglomérés, nombreus, arror. dis, inégaux, sont disseminés partout dans la peau, et sont contenus entre l'épiderme et la couche musculaire qui est dessous, et entre les fibres mêmes de la dite couche. Moquin-Tandon a observé que ces grains forment des plaques irrégulières autour du tortillon du mollusque; ces dépôts calcaires sout destinés à fournir les éléments nécessaires à l'augmentation et à l'entretien de l'enveloppe testacée.

L'opercule est composé de trois couches, dont l'externe, très mince, n'est qu'un épiderme homogène, à plusieurs plis correspondants à autant de stries d'accroissement dans l'opercule; la couche ou assise médiane, beaucoup plus épaisse, est calcaire; la troisième, ou intérieure, est cornée, brunâtre, sans structure apparente; plus épaissie vers la région centrale que dans toutes les autres parties de l'opercule. La face interne de cette troisième couche est revêtue d'un épithélium cellulaire; mais le plus souvent il n'existe que sur les bords de l'opercule, et bien loin du centre spiral; les cellules sont polygonales, plus ou moins allongées, et chacune contient un nucléole central étroitallongé ou bien ovale-arrondi (1).

Système sensitif. - Le C. elegans possède douze ganglions, dont buit appartiennent au système nerveux central, deux all sympathique, et les

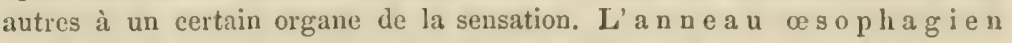
est formé de six ganglions; les deux supérieurs (sus-œ s o phag i ens a n téri e urs), piriformes, sont situés près du pharynx, au-dessus de l'œeso. phage. De ces ganglions partent plusieurs nerfs, et se dirigent les uns aux organes des sens, les autres aux pharynx et aux régions voisines. $\Lambda$ la place du petit anneau sous-œsophagien qui existe chez plusieurs gastéropodes, on y voit deux ganglions claviformes (ganglia pedalia); de ces ganglions partent plusieures petites ramifications nerveuses qui vont jusqu'au pied, non seulement, mais produisent aussi le nerf acustique. La ligne de conjonction entre le ganglion pédal et le ganglion latéral à droite, est plus courte de celle qui unit le ganglion du pied au ganglion latéral senestre; d'où en resulte que l'anneau osophagien est formé de trois paire de ganglions, l'une supérieure, un'autre latérale, l'autre inférieure.

Le système sympathique, ou viscéral nerveux, est formé de deux petits ganglions situés sous le pharynx, près de l'axe longitudinal du corps.

Des organes de l' o u ïe nous avons déjà parlé; nous ajouterons seulement que les deux poches ou capsules auditives sont entourées d'une couche de

(5) M. Claparide n'alopte pas I'opinion de quelques auteurs qui pensent que lous les opercules cochliuformes (c'est a dire a strie spirale) sont produits par les hords dı manteau. 
cellules demi-transparentes (1) avec un nucléole central (2); et l'otolithe (unique) sphérique contenue dans chaque capsule est formée de couches concentriques et très réfringentes la lumière. Quelques-unes des dites cellules sont pleines quelquefois de nombreux petits grains, eux aussi très réfringents la lumière, et il y a même des cellules entre les organes de la reproduction, lesquelles contiennent une substance granuleuse et jaunâtre.

Le globe oc ula ire du $C$. elegans est sphérique, et on y distingue assez bien la cornée et la sclérotique. La face interne de la sclérotique est tapissée par la membrane choroïdée composée de cellules pleines de matière colorante. La r ét in e, qui occupe le fond de la chambre postérieure, est elle aussi une membrane cellulaire d'une ténuité excessive, et dont les cellules se dissolvent très facilement. L'intérieur du globe oculaire contient le $\mathrm{c}$ r istallin lenticulaire et l'humeur vitrée, mais ni l'un ni l'autre n'ont une structure apparente.

Système digestif - Des cartilages et des dents linguales nous avons déjà fait mention lorsque nous avons parlé des organes ingestifs des mollusques operculés. Le ruban lingual du C. elegans (de même que celui du Pomatias, de tous les Rhipidoglosses et de plusieurs autres mollusques) est dilaté antérieurement, et divisé en deux ailes. Le p al a is est recouvert. d'un épithélium formé de cellules oblongues, hexagonales, et plus ou moins larges ou sub-arrondies. Ces cellules épithéliales sont demi-cornées, et offrent beaucoup d'affinité avec la membrane palatine dont sont pourvues les Helices, outre leur mâchoire.

L'œ s o p h a g e, dont la longueur est de dix à douze millimètres, est situé près de la ligne médiane du corps, et se rend dans l'e s t o m a c. Celui-ci est oblong et fusiforme; sa terminaison postérieure se présente en forme de petit cul-de-sac (3); l'antérieure se prolonge dans l'intestin; la partie aveugle de l'estomac est entourée par le foie.

$L$ 'in test in recourbé et entortillé, arrive enfin au bord droit du rein (4) et d'ici par une voie toute droite se dirige par dessous la cavité pulmonaire jusqu'à l'a nus. L'ouverture anale est située près de l'ouverture sexuelle, sous le bord droit de la cuirasse. La paroi du canal ou tube intestinal est composée de trois couches: la plus interne est un épithélium ciliaire qui se rencontre aussi dans le tube digestif; la couche médiane est de nature musculaire; la troisième, ou extérieure, est composée de cellules (5), d'une sub-

(1) Tela conjunctiva, Leydig.

(2) Des cellules semblables existent même dans les tortillons de l'intestin, dans la région de l'estomac et de l'ovaire, et parmi les glohules du foie.

(3) Ventriculus inferior sive cocus, Lister.

(4) Glande précordiale, Moquin-Tandun.

(5) Cellules conjonclives. 
stance graisse (1). Le pavement musculaire de l'estomac est très grand, et on peut facilement le séparer des autres couches, et même y reconnaitre les fibres circulaires. Bien différentes sont les cellules du réseau vasculaire de l'estomac, et contiennent une matière brunâtre; on rencontre des cellules identiques dans la région du réseau vasculaire de la cavité respiratoire, et même dans quelques autres cavités du corps. Tout le long de l'intestin, entre les cellules épithéliales, existent des glandules nombreuses, fusiformes (2); mais sans nucléole central; ont pent cependant supposer qu'elles soient plutôt de vraies cellules que d'espaces intercellulaires. Par une légère compression, la substance contenue dans les glandules intestinales se présente sous la forme de corpuscules sphériques, mais les grains contenus dans les glandes de l'estomac et de l'œesophage ressemblent à une bouteille à long col.

Les g landes salivaires sont représentées par deux petits corps blancs et oblongs; la partie postérieure est plus épaisse, l'antérieure est plus étroite et sinueuse; vers l'extrémité elle se rétrécit et devient enfin subulée. Cette glande passe par l'anneau nerveux oesophagien, et par l'œesophage même penètre daus la cavité pharyngéenne. Les glandes salivaires sont creuses à l'intéricur sur toute la longueur, et par ce canal entre un grand nombre de follicules; ce même canal reçoit la substance sécrétée par les follicules sous la forme de très petits grains, contenus quelquefois dans des cellules, et quelquefois libres par suite de la dissolution des cellules mêmes lesquelles ne sont enfin autre chose que l'épithélium des follicules. Le foic change de volume suivant les temps et les conditions de l'animal. Trois sont les éléments ou les substances produites par les cellules hépatiques du C. elegans; tandis qu'il n'y en a que deux chez tous les autres mollusques examinés jusqu'ici. Et d'abord, en observant même à l'œeil nu les follicules, on y voit assez bien des petits points d'un brun-roussâtre, dans les quels, à l'aide d'un bon microscope, sont visibles les agglomérations de la matière colorante biliaire; plusieurs de ces globules sont spbériques, mais il y en a qui présentent une forme irrégulière, ou bien qui sont entourés d'une couche extérieure plus luisante et jaunatre-pâle; il arrive aussi quelquefois d'observer ces globules coloriés placés dans des cellules nucléolées, et alors la couche jaunâtre est plus pâle, et a l'apparence d'une liqueur contenue dans une membrane très-mince (3). Lorsque la matière colorante de la bile du C. elegans est traitée par les alcalis (ammoniaque), la vésicule qui la contient est détruite, la matière se dissout sans cependant changer de couleur, et se

(1) Substuntia conjuncliva, Leydig.

(2) Ces glandes existent aussi dans l'estomac ef dans l'épidertne exterieur.

(3) C'est leut-ètre celte membrane que Heckel a appellée siek retbläs ch en, ou lesirule de la matirre serétée. 
transforme ensuite en cristaux jaunes ou incolores; traitée cette même sub. stance colorante par les acides (hydrochlorique) ils ne font pas verdir les globules brunâtres du foie, mais les dissolvent en manière que la membrane dont ils sont enveloppés ne vient pas altérée. Le contraire arrive chez presque tous les autres mollusques (1): la matière colorante de leur bile soumise à l'action des alcalis, prend une teinte plus foncée, et par l'action des acides devient un peu verdâtre (Limnées, Planorbes, etc.), ou verte (Heliées, etc.). Chaque globule biliaire du foie, chez le C. elegans, est enveloppé dans une membrane assez épaisse et brunâtre; cette membrane sert le plus souvent d'enveloppe à une autre vésicule plus petite qui sépare la couche jaune extérieure de la substance médiane de couleur brune. On pourrait bien dire donc d'avoir ici deux cellules Meckelianes, l'une contenue dans l'autre.

L'autre des trois éléments, ou substances, des cellules hépatiques est composé de vésicules incolores, pleines de globules arrondis et de toutes dimensions, presentant sous l'action des réactifs chimiques les mêmes caractères des globules colorants de la bile. Le troisième élément enfin est représenté par des globules arrondis et adipeux, qui ne sont pas enveloppés dans des cellules, et ont la propriété de réfracter les rayons du soleil. De tuut ce que nous avons dit s'ensuit que le $C$. elegans serait dépourvu de vraie bile, par la raison que la bile ne peut autrement se former que par une seconde liquefaction des globules de la matière colorante biliaire. Le foie du C. elegans est tout enveloppé dans une membrane musculeuse et mince, laquelle, en effet, appartient à la couche musculeuse qui fait part du manteau.

Système circulatoire - Le c œu $\mathrm{r}$ de notre Cyclostome est placé du côté droit, vers la partie inférieure de la cavité respiratoire, et près du rein. Les $\mathrm{va}$ is se a $\mathrm{x}$ s a $\mathrm{ng}$ ins ne possèdent pas une paroi leur propre; il ne sont représentés que par des conduits ou canaux ramifiés, placés entre les organes du mollusque. Le sang n'est autre chose qu'une liqueur limpide dans le quel sont contenus plusieurs corpuseules.

Système sécrétoire - Le rein (2), est une glande triangulaire (3), de couleur vert-olivâtre, attachée au plafond de la cavité respiratoire, du côté droit de l'intestin rectum, et devant les tortillons intestinaux; la partie interue, ou la plus profonde, du rein se trouve près du cœur. Les cellules rénales sont des vésicules arrondies et limpides; les calculs rénaux ne sont pas arrondis et simples comme ceux de plusieurs autres mollusques pulmonés, mais ont la forme de petits corpuscules jaunes, agglomérés et inégaux. L'bumeur produite

(1) Chez les Limnées, les Planorbes, les Paludines, les Helicées et méme chez les Dreissenides, les Cyclades, etc. (Meckel).

(2) Glande précurdiale, Moquin-Tandon.

(3) Moquin-Tandon dit que celte glande est ovalaire. 
par le rein peut être régardée comme l'urine du mollusque. Les cellules urinaires sont disposées en plusieures assises, les unes superposées aux autres de manière que les cellules plus grandes sont dessus, et les plus petites dessous.

Mais ce qu'il y a de remarquable chez le C. elegans est la présence d'un organe tout à fait spécial qui n'a pas été encore observé ni dans les Pomatias, ni dans les Ampullaires, quoique alliés des Cyclostomes, ni enfin dans les autres mollusques. Cet organe est une glande assez volumineuse placée entre les tortillons de l'intestin et pénétrant entre le rein, le cœur et la cavité respiratoire, et sans ouverture quelconque. Les lobes de cette glande, touchant la face extérieure des replis intestinaux, se dessinent assez nettement sous la forme d'une ligne blanche à angles alternes, même en dehors sur le dos de l'animal (lorsqu'on l'a tiré de sa coquille). Cet organe est formé d'un grand nombre de petits grains arrondis, opaques, ì couches concentriques et membraneuses (1); ils sont d'un beau blanc luisant par la réflexion des rayons de la lumière, et par les rayons lumineux incidents ces grains ressemblent à des petites étoiles luisantes sémées dans un ciel obscur. D'après l'analyse chimique, ces petits corpuscules granuleus seraient composés, en grande partie, de calcaire; très probablement cet organe a une fonction excrétoire, et comme accessoire ì la fonction rénale. Moquin-Tandon n'a pas connu cette glande, on bien l'a confondue arec sa glande précorliale.

Nous dirons enfin quelques mots sur la nature d'un autre organe sécrétoire, on plutôt excrétoire, qui a été déjà observé par MM.rs Delle Chiaje et Klebeerg chez divers mollusques pulmonés, mais qui dans le C. elegans a une structure bien différente. Sous la pean, entre le bouche et le pied, existe une espèce de capsule, ou sac ovale et large, pleine d'une matière blanche, visible même en dehors, sur la peau. De ce petit sac partent deux conduits tubulaires, longs, repliés en diverses façons, et formant une espèce de peloton autour des ganglions sous-œesophagiens et des vésicules auditives; l'épithélium de ces tuyaux est cellulaire, et les cellules sécrètent une substance consistante en petits grains arrondis. Ces cellules poussées dans l'intérieur du tuyau, entrent dans le sac; quant à la substance ou mucosité sécrétée, elle paraît destinée à écouler devant le pied du mollusque, lorsqu'il est en marche, pour en lubrifier le passage. Dans le dit sac existent aussi d'autres cellules de la même dimension, mais la matière qu'elles contiennent n'est pas de la même nature; ce sont de très petits grains toujours tremblotants (2).

(1) Ces petits grains opaques traites par l'ammoniaque et la potasse caustique, perdent leur substance organique, et deviennent peu à peu translucides de la périphérie au centre; les couches concentriques sont plus visibles si, au lieu de l'ammoniaque, on fait usage d'acide hydrochlorique.

(2) Motio molecularis, Brown. 


\section{$-141$}

Système reproducteur. - Les follicules testiculaires sont enveloppés d'une membrane sans structure apparente; la face interne de cette membrane est couverte de cellules épithéliales pourvues d'un nucléole central, et colorées en jaune. Dans la cavité des follicules il y a des cellules à noyau assez volumineux, quelquefois double et comme étranglé au milieu ; et plusieures cellules contiennent même trois ou quatre nucléoles. Existent aussi des globules nombreus qui ont toute l'apparence des nucléoles dont nous avons parlé à présent, et ces globules se changent en cellules productrices des zoospermes. Enfin on y voit des cellules à contour bien determiné, lesquelles contiennent une substance granuleuse; ces cellules ne sont autre chose que des nucléoles libres des cellules épithéliales renfermantes la matière colorante jaune. Les petits nucléoles, dans les nouyaux des cellules primitives, se changent en nucléoles de cellules zoospermiques, et eux mêmes en zoospermes. Le c a $\mathbf{n}$ a 1 d éf ér e n t est revêtu d'un épithélium cellulaire, à cellules pourvues de nucléole central. Le prostate est une capsule volumineuse, dont la paroi épaisse et glanduleuse est formée (comme chez les autres mollusques) de plusieurs plis ou lames presque parallèles; ces lames produisent de très petits corpuscules granuleux et incolores, inclus dans des cellules arrondies. L'o v a ir e consiste en une glande formée de plusieurs follicules, et enveloppée dans une pellicule très mince qui passe d'un follicule à l'autre; chaque follicule est revêtu d'un épithélium pavé à cellules hexagonales, dans lesquelles existe un noyau central, ou bien un peu subcentral, contenant lui même un petit nucléole. L'o vi ducte, plié et réplié, est uni par sa partie inférieure (à cet effet un peu dilatée) à un organe spécial ou corps vermiforme, plus étroit devant qu'en arrière, et placé sous la cavité respiratoire, du côté droit, et sous l'intestin; la partie postérieure est lisse, l'antérieure est ornée de stries circulaires et transversales. De la $\mathrm{m}$ a trice nous dirons seulement que la paroi interne de sa partie inférieure présente une structure foliacée ou lamelleuse, et les lames sont couvertes de cellules qui sécrètent des petits grains; les uns et les autres solubles dans les alcalis. La partie supérieure est lisse et non foliacée, et contient une liqueur épaisse, formée de corpuscules nombreux, ovales et plans, et de couleur pâle. 

PLANCHE I. 


\section{PLANCHE 1.}

1. Limax cinereo-niger, Wolf - a. Animal raccourci, vu en dessus b. Fragment de la sole.

2. - $\quad$ Variété? - a. Fragment dorsal - b. Fragment de la sole.

3. Succinea Droueti, Dumont et Mortillet - $a$. Coquille considérablement grossie, vue de face $-b$. La même, vue par le dos $-c$. Coquille de grandeur naturelle.

4. - hustus, Drouët - a. Coquille considérablement grossie, vue de face $-b$. La même, vue par le dos $-c$. Coquille de grand. nat.

5. Helix unbulicaris, Brumati; Var. Padana - a. Coquille de grand. nat., vue de face - $b$. La même, vue en dessus - $c$. La mème, vue en dessous $-d$. Fragment, considérablement grossi, du dernier tour, pour faire voir les granulations de l'épiderme et leur disposition.

6. - poetens, Studer, Var. Cisalpina - $a$. Coquille de grand. nat., vue en dessus - b. La mème, vue de face - c. La mème, vue en dessous. 

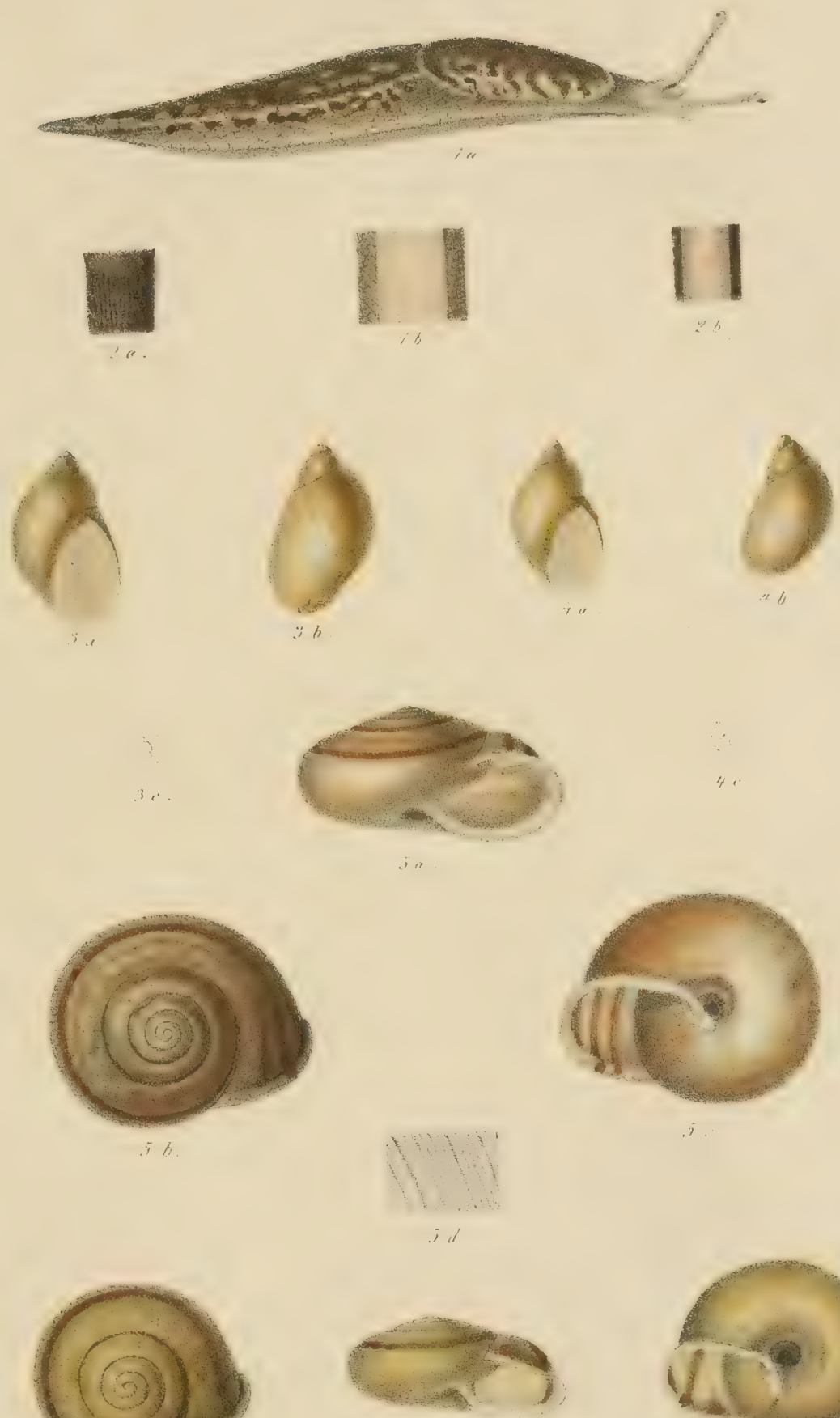

sis

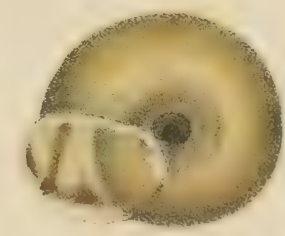

ii 

PLANCHE II. 


\section{PLANCHE II.}

1. Clausilia Melle, Stabile. Coquille considérablement grossie verture vue de face $-b$. Dernier tour vu par le dos, pour montrer la gibbosité, la lunelle et le pli palatal - c. Le même vu de profil - $d$. Coquille grandeur de naturelle, vue de face.

2. - Verbanensis, Stabile. Coquille consid. grossie - a. Ouverture vue de face $-b$. Dernieur tour vu par le dos, pour montrer les plis palataux $-c$. Le même vu de profil $-d$. Coquille de grand. nat., vue de face.

3. - alpisa, Stabile. Coquille consid. grossie - a. Ouvert. vue de face $-b$. Dernier tour vu par le dos $-c$. Le même vu de profil - $d$. Coquille de grand. nat., vue de face; avec les sutures papillifères.

4. Pupa Mortilleti, Stabile. Coquille consid. grossie - a. Ouvert. vue de face - b. Dernier tour vu par le dos, pour montrer les plis palataux - c. Coq. de grand. nat., vue de face.

5. - multidentata (Turbo), Olivi. Coquille consid. grossic - a. Ouverture vue de face $-b$. Dernier tour vu par le dos $-c$. Coquille de grand. natur., vue de face.

Cette dernicre espice a itć figuré pour servir de termo de comparaison. 


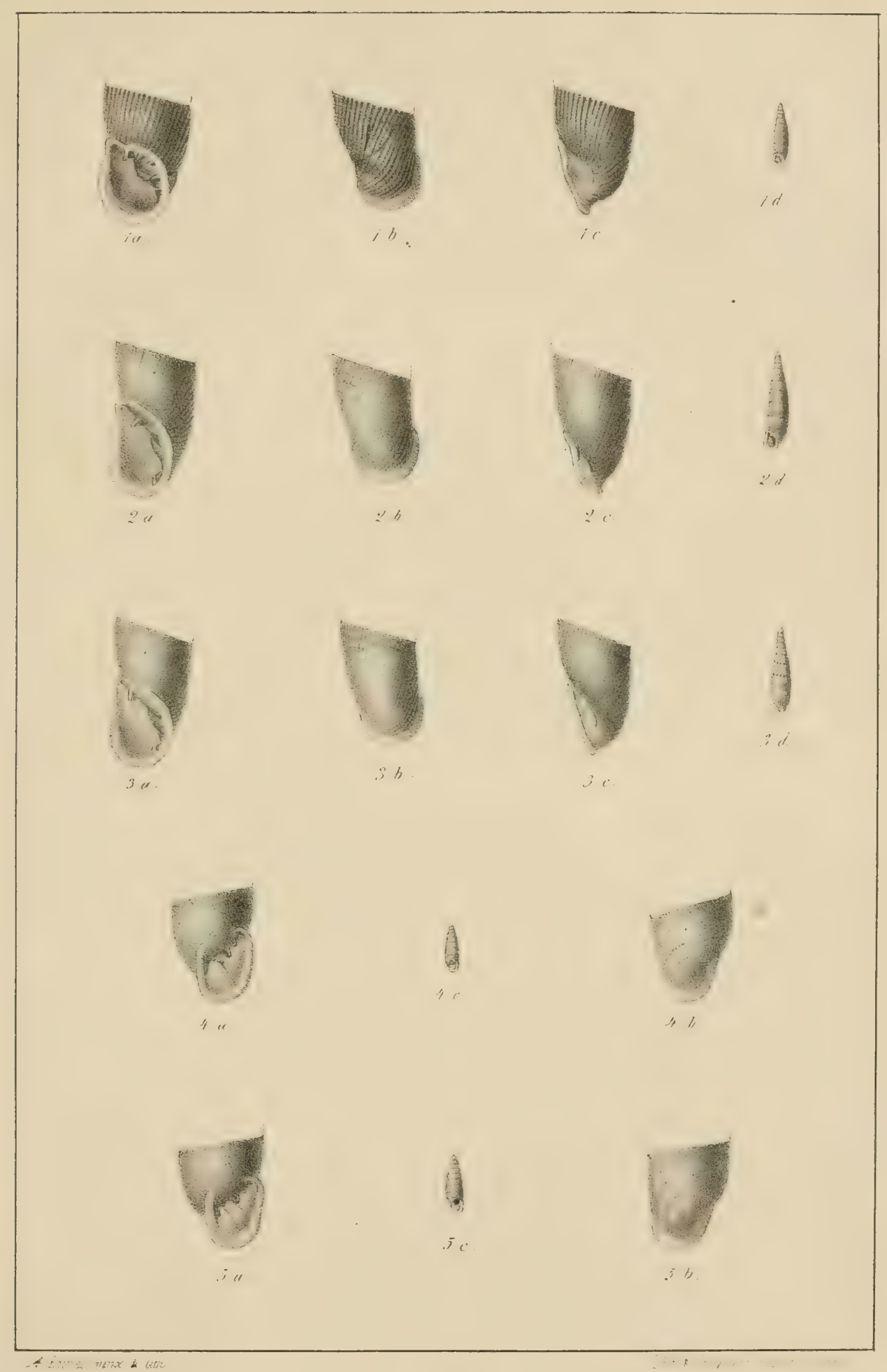






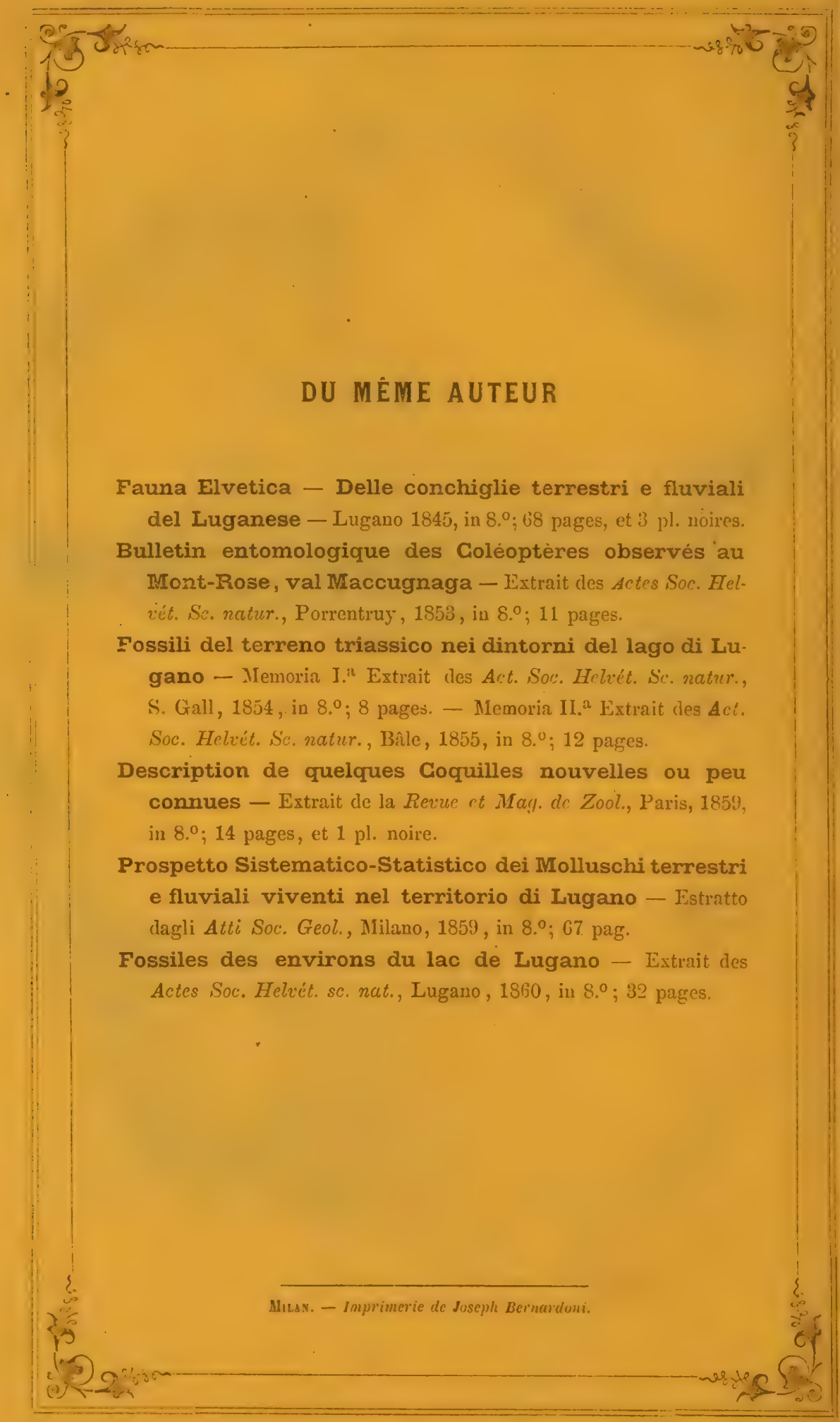





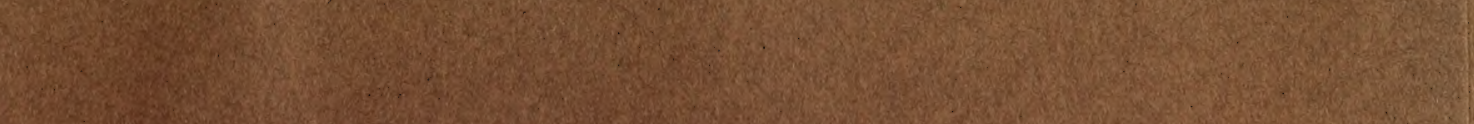

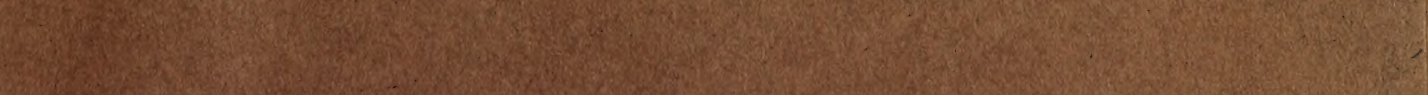
Q6.

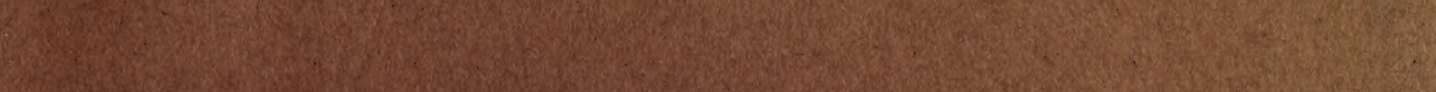
A3)

?. -19.8. . 

W.

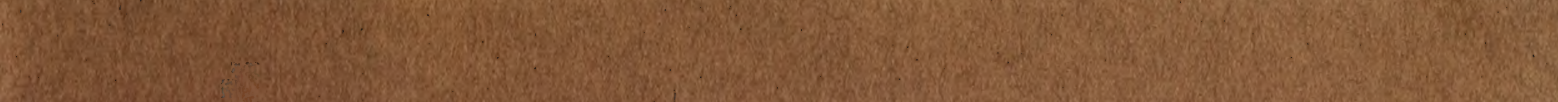

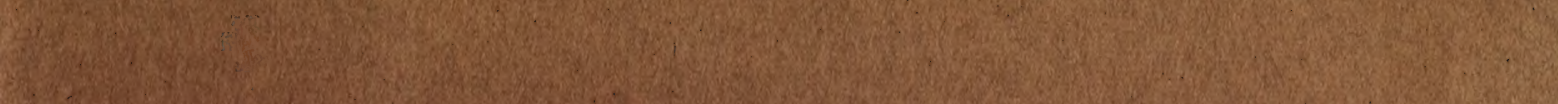

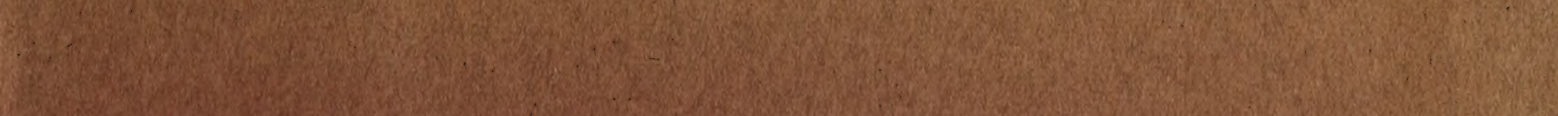

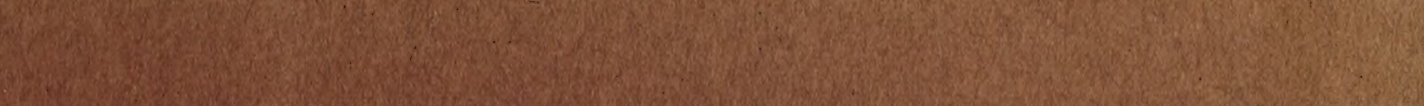

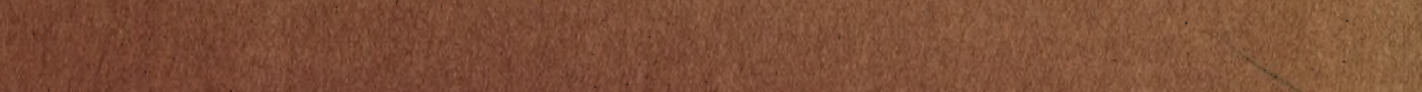
w 2.4.

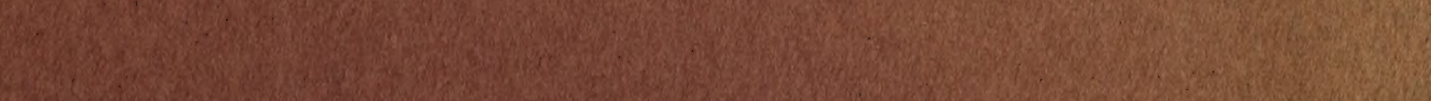
2.

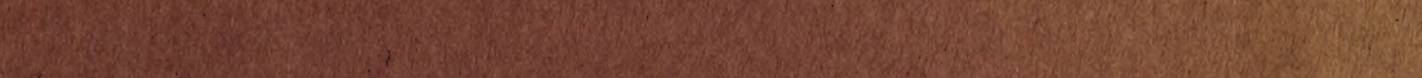
W. W.

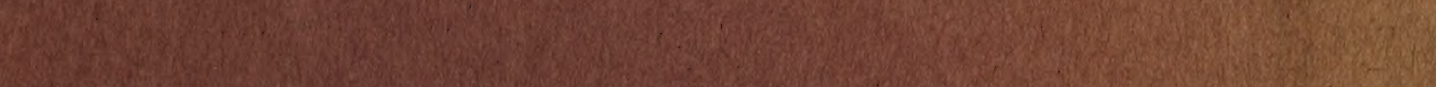
(3)

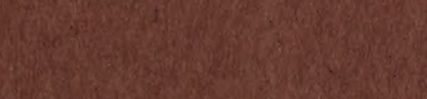
(4)

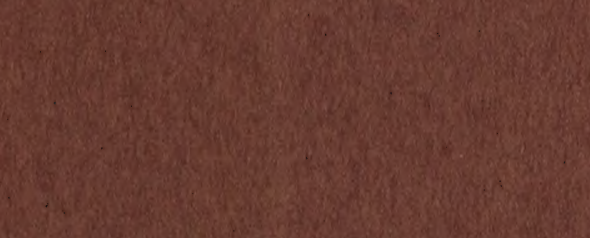

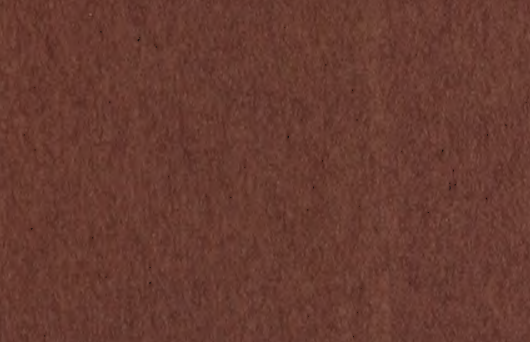

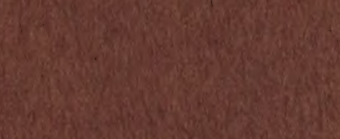

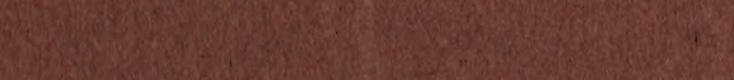

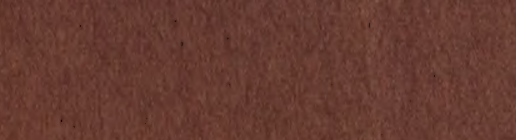

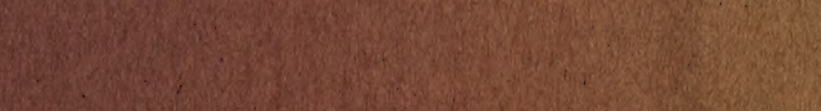

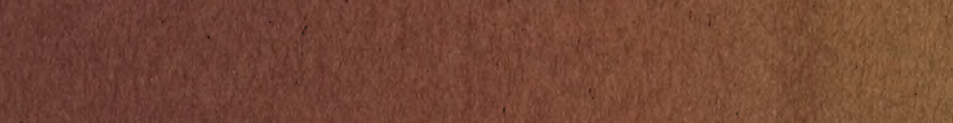
2.

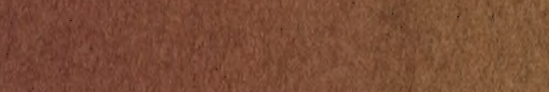

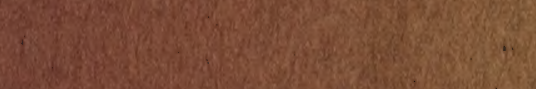

(6)

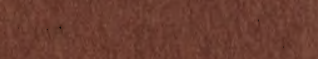

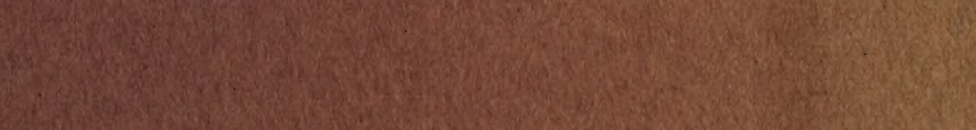
S.

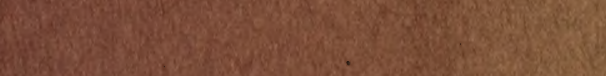

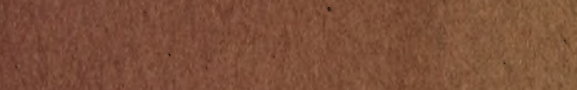
Q5is.

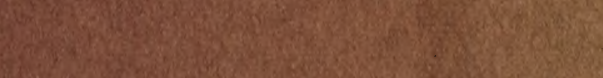

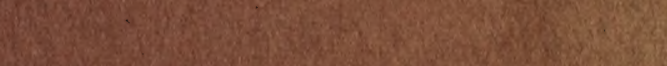

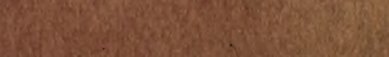

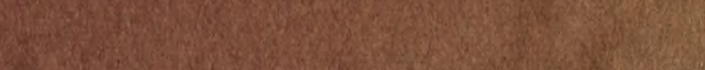
S. Who 46 
